\title{
THE PREDICTION OF PASSENGER RIDING COMFORT FROM ACCELERATION DATA
}

CRAIG C. SMITH

DAVID Y. MCGEHEE ANTHONY J. HEALEY

\section{RESEARCH REPORT 16}

\author{
MARCH 1976
}
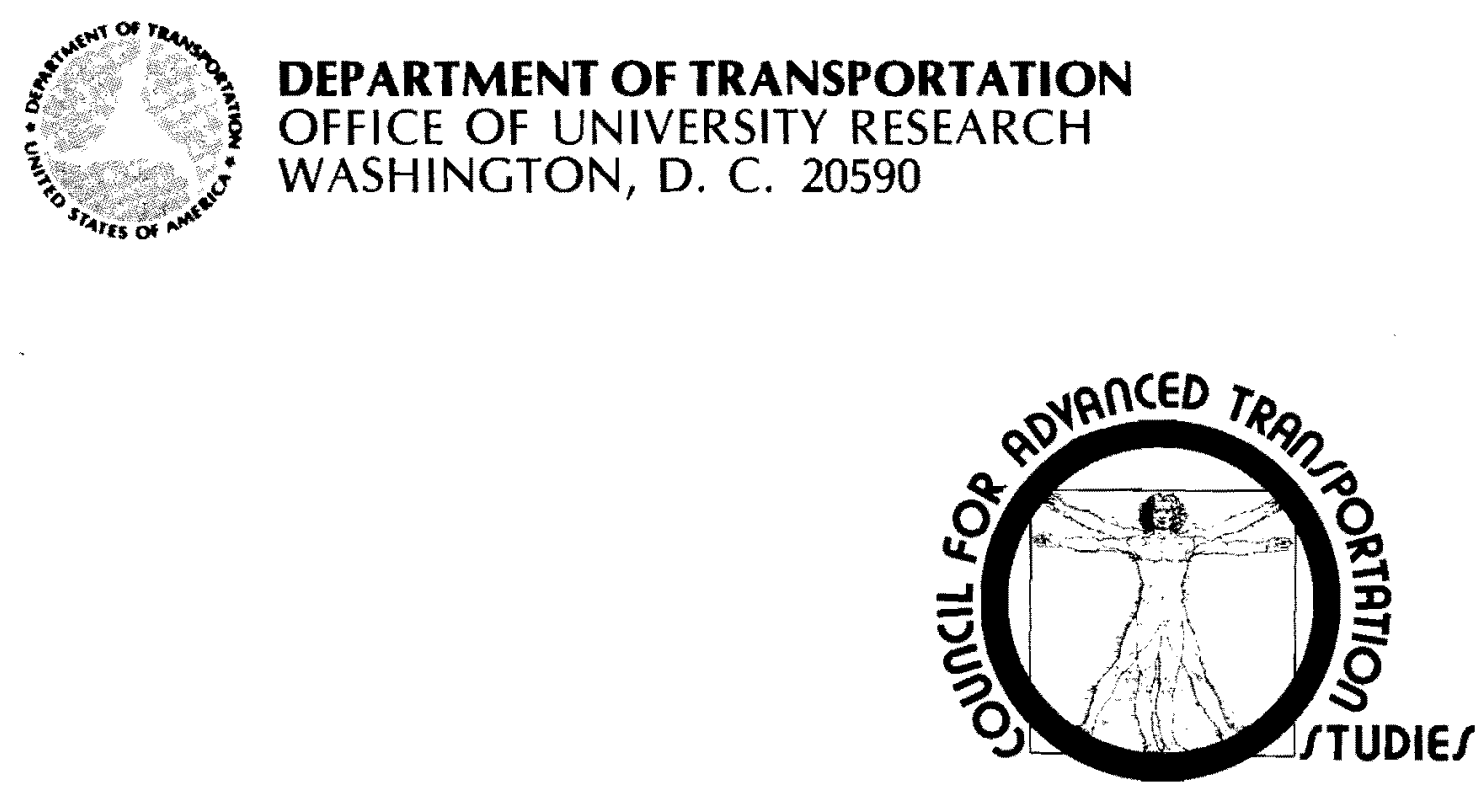


\section{RESEARCH REPORTS PUBLISHED BY \\ THE COUNCIL FOR ADVANCED TRANSPORTATION STUDIES}

1 An Integrated Methodology for Estimating Demand for Essential Services with an Application to Hospital Care. Ronald Briggs, Wayne T. Enders, James Fitzsimmons, and Paul Jensen, April 1974 (DOT-TST-75-61).

2 Transportation Impact Studies: A Review with Emphasis on Rural Areas. Lidvard Skorpa, Richard Dodge, C. Michael Walton, and lohn Huddleston, October 1974 (DOT-TST-75-59).

4 Inventory of Freight Transportation in the Southwest/Part I: Major Users of Transportation in the Dallas-Fort Worth Area. Eugene Robinson, December 1973 (DOT-TST-75-29).

5 Inventory of Freight Transportation in the Southwest/Part II: Motor Common Carrier Service in the Dallas-Fort Worth Area. J. Bryan Adair and James S. Wilson, December 1973 (DOT-TST-75-30).

6 Inventory of Freight Transportation in the Southwest/Part III: Air Freight Service in the Dallas-Fort Worth Area. J. Bryan Adair, June 1974 (DOT-TST-75-31).

7 Political Decision Processes, Transportation Investment and Changes in Urban Land Use: A Selective Bibliography with Particular Reference to Airports and Highways. William D. Chipman, Harry P. Wolfe, and Pat Burnett, March 1974 (DOT-TST-75-28).

9 Dissemination of Information to Increase Use of Austin Mass Transit: A Preliminary Study. Gene Burd, October 1973.

10 The University of Texas at Austin: A Campus Transportation Survey. Sandra Rosenbloom, Jane Sentilles Greig, and Lawrence Sullivan Ross, August 1973.

11 Carpool and Bus Matching Programs for The University of Texas at Austin. Sandra Rosenbloom and Nancy Shelton Bauer, September 1974.

12 A Pavement Design and Management System for Forest Service Roads: A Conceptual Study. W. R. Hudson and Thomas G. McGarragh, July 1974.

13 Measurement of Roadway Roughness and Motion Spectra for the Automobile Highway System. Randall Bolding, Anthony Healey, and Ronald Stearman, December 1974.

14 Dynamic Modeling for Automobile Acceleration Response and Ride Quality Over Rough Roadways. Anthony Healey, Craig C. Smith, Ronald Stearman, and Edward Nathman, December 1974.

15 Survey of Ground Transportation Patterns at the Dallas-Fort Worth Regional Airport. William J. Dunlay, Jr., Thomas G. Caffery, Lyndon Henry, and Douglas Wiersig, August 1975.

16 The Prediction of Passenger Riding Comfort from Acceleration Data. Craig C. Smith, David Y. McGehee, and Anthony I. Healey, March 1976.

17 The Transportation Problems of the Mentally Retarded. Shane Davies and John W. Carley, December 1974.

18 Transportation-Related Constructs of Activity Spaces of Small Town Residents. Pat Burnett, John Betak, David Chang, Wayne Enders, and Jose Montemayor, December 1974 (DOT-TST-75-135).

19 Marketing of Public Transportation: Method and Application. Mark 1. Alpert and Shane Davies, January 1975.

20 The Problems of Implementing a 911 Emergency Telephone Number System in a Rural Region. Ronald T. Matthews, February 1975.

23 Forecast of Truckload Freight of Class / Motor Carriers of Property. Mary Lee Gorse, March 1975 (DOT-TST-75-138).

24 Forecast of Revenue Freight Carried by Rail in Texas to 1990. David L. Williams, April 1975 (DOT-TST-75-139).

28 Pupil Transportation in Texas. Ronald Briggs, Kelly Hamby, and David Venhuizen, July 1975.

30 Passenger Response to Random Vibration in Transportation Vehicles. Anthony J. Healey, June 1975.

35 Perceived Environmental Utility under Alternative Transportation Systems: A Framework for Analysis. Pat Burnett, March 1976.

36 Monitoring the Effects of the Dallas/Fort Worth Regional Airport. Volume I: Ground Transportation Impacts. William I. Dunlay,

Ir., Thomas G. Caffery, Lyndon Henry, Douglas W. Wiersig, and Waldo Zambrano, December 1976.

37 Monitoring the Effects of the Dallas/Fort Worth Regional Airport. Volume II: Land Use and Travel Behavior. Pat Burnett, David Chang, Carl Gregory, Arthur Friedman, Jose Montemayor, and Donna Prestwood, July 1976.

38 Transportation and Community Development-A Manual for Small Communities: Level I, Volume 1-Executive Summary; Volume II-The Planning Process. Richard Dodge, John Betak, C. Michael Walton, Charles Heimsath, and John Huddleston, July 1976.

39 An Evaluation of Promotional Tactics and Utility Measurement Methods for Public Transportation Systems. Mark Alpert, Linda Golden, John Betak, James Story, and C. Shane Davies, March 1977.

40 A Survey of Longitudinal Acceleration Comfort Studies in Ground Transportation Vehicles. L. L. Hoberock, July 1976.

41 Lateral Steering Dynamics Model for the Dallas/Fort Worth AIRTRANS. Craig C. Smith, December 1976 (Draft Report).

42 Guideway Sidewall Roughness and Guidewheel Spring Compressions of the Dallas/Fort Worth AIRTRANS. William R. Murray and Craig C. Smith, August 1976 (Draft Report).

43 A Pavement Design and Management System for Forest Service Roads: A Working Model. Freddy L. Roberts, B. Frank McCullough, Hugh J. Williamson, William R. Wallin, February 1977.

44 A Tandem-Queue Algorithm for Evaluation of Overall Airport Capacity. Chang-Ho Park, April 1977 (Draft Report).

45 Characteristics of Local Passenger Transportation in Texas. Ronald Briggs, January 1977 (Draft Report). 
THE PREDICTION OF PASSENGER RIDING COMFORT

FROM ACCELERATION DATA

Craig C. Smith

David Y. McGehee

Anthony J. Healey

March 1976

RESEARCH REPORT

Document is available to the public through the National Technical Information Service, Springfield, Virginia 22151

\author{
Prepared for \\ COUNCIL FOR ADVANCED TRANSPORTATION STUDIES \\ THE UNIVERSITY OF TEXAS AT AUSTIN \\ AUSTIN, TEXAS 78712 \\ In cooperation with \\ U. S. DEPARTMENT OF TRANSPORTATION \\ OFFICE OF UNIVERSITY RESEARCH \\ WASHINGTON, D. C. 20590
}




\section{NOTICE}

This document is disseminated under the sponsorship of the Department of Transportation, of fice of University Research, in the interest of information exchange. The United States Government and The University of Texas at Austin assume no liability for its contents or use thereof. 


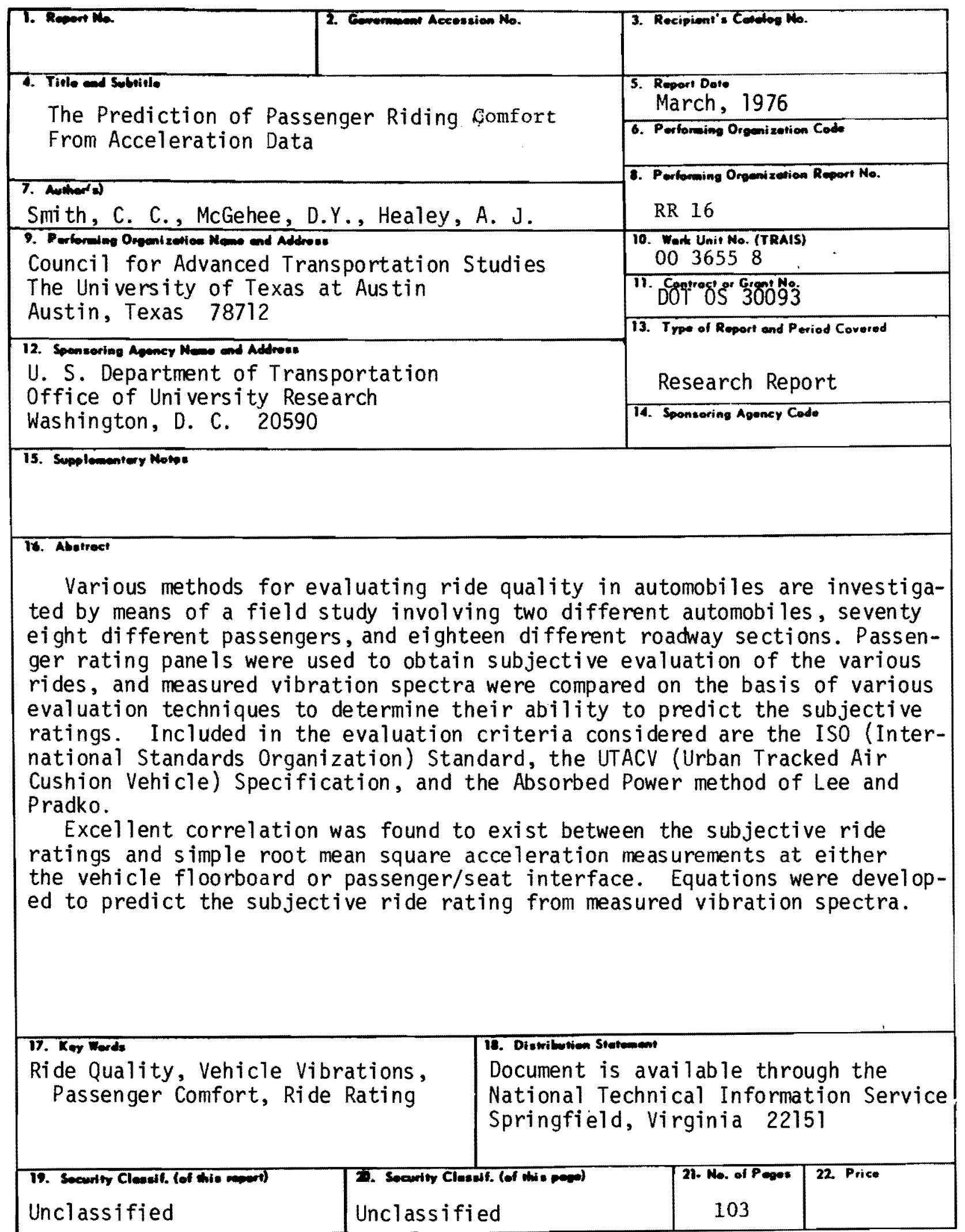




\section{EXECUTIVE SUMMARY}

\section{Abstract}

Various methods for evaluating ride quality in automobiles are investigated by means of a field study involving two different automobiles, seventy-eight different passengers, and eighteen different roadway sections. Passenger rating panels were used to obtain subjective evaluation of the various rides, and measured vibration spectra were compared on the basis of various evaluation techniques to determine their ability to predict the subjective ratings. Included in the evaluation criteria considered are the ISO (International Standards Organization) Standard, the UTACV (Urban Tracked Air Cushion Vehicle) Specification, and the Absorbed Power method of Lee and Pradko.

Excellent correlation was found to exist between the subjective ride ratings and simple root mean square acceleration measurements at either the vehicle floorboard or the passenger/seat interface. Equations were developed to predict the subjective ride rating from measured vibration spectra.

\section{Introduction}

The acceptance of any new transportation system is affected by the vibrations or "ride quality" to which passengers are exposed. Study of the response of passengers to a vehicle vibration environment has therefore become increasingly important to assist in the development of these new systems. While underdesign of a system with regard to allowed vibration levels can cause it to be unacceptable to the traveling public, overdesign can lead to excessive system costs.

It is therefore imperative that we understand the relationships between allowed vibration levels and passenger acceptance. This study is a detailed study of these relationships for the automobile, a presently well accepted mode of transportation within the common experience of the traveling public. This provides a baseline comparison for examination of other, newer modes of transportation. 
Method

The collection of data for the study here described included the measurement of passenger subjective response (ratings) to a variety of riding vibrations in different automobiles over different roadways. Acceleration measurements of the corresponding vibrations were also recorded, including both vertical and lateral floorboard and vertical and lateral seat/passenger interface accelerations. The subjective (passenger ratings) and objective (acceleration measurements) measures of the ride were then compared via a variety of proposed ride rating methods to determine the method which best predicts the subjective ratings using the objective measurements. Methods compared include the ISO Standard, the UTACV Specification, the Absorbed Power method of Lee and Pradko, and frequency weighting techniques utilizing various proposed curves relating human sensitivity to vibration as a function of frequency.

\section{Findings and Results}

A variety of the frequency weighting schemes investigated relate reasonably well to the subjective passenger responses. Of these, the simple r.m.s. acceleration measures, the simplest to use, are also consistently as good of predictors as the more elaborate schemes. Measured at the floorboard, the vertical r.m.s. acceleration is an excellent predictor, while at the Dassenger/seat interface, the lateral r.m.s. acceleration is the better predictor. The magnitude (defined as the square root of the sum of the squares of the vertical and lateral r.m.s. values) acceleration is a good predictor for either the floorboard or passenger/seat interface vibrations. 
This work was supported by the U. S. Department of Transportation, University Research Office, under contract DOT-0S-30093, administered through The Council for Advanced Transportation Studies at The University of Texas at Austin.

Also of significant help to the project was the loan of a portable three axis accelerometer package by the Nasa-Langley Research Center and the support of the Texas State Department of Highways and Public Transportation and The University of Texas Center for Highway Research in identifying the highway test sections used. 
NOMENCLATURE

INTRODUCTION

1.1 Existing Criteria 2

1.2 Objectives and Scope of Study 4

MEASUREMENT AND PROCESSING OF ACCELERATION DATA 6

2.1 Measurement of Accleration Data 6

2.2 Digitizing of Data 8

$\begin{array}{lll}2.3 & \text { Spectral Density Calculations } & 10\end{array}$

2.4 Weighted RMS Acceleration "ride index" Calculations 10 PSYCHOMETRIC EXPERIMENTS FOR RIDE EVALUATION 12

3.1 Buick Rating Sessions 12

3.1.1 General 12

3.1.2 Routes 12

3.1.3 Design 13

3.1.4 Subjects 13

3.1 .5 Procedure 13

3.1 .6 Results $\quad 14$

3.2 Maverick Rating Session (Fall 1975) 14

3.2.1 General 14

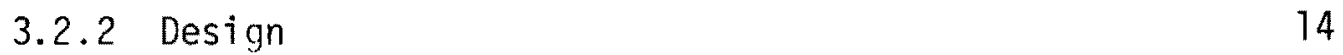

3.2.3 Sensory Modes 15

$\begin{array}{lll}3.2 .4 & \text { Subjects } & 15\end{array}$

$\begin{array}{lll}3.2 .5 & \text { Procedure } & 15\end{array}$

$\begin{array}{lll}3.2 .6 & \text { Results } & 16\end{array}$ 
COMPARISON OF PASSENGER RATINGS WITH ISO AND UTACV SPECIFICATIONS

4. 1 Comparison with UTACV Boundaries

4.2 Comparison with ISO Boundaries 20

FREQUENCY WEIGHTED RIDE INDICES AND THEIR CORRELATION WITH MEAN PERSONAL RATIINGS

5.1 Weighting Functions 22

5.2 Absorbed Power 28

5.3 Unweighted RMS Acceleration Ride Indices 31

5.4 Correlation Study 31

5.5 Discussion of Statistical Results 32

5.5.1 Frequency Weighting Versus Unweighted Values 32

5.5.2 Significance of Location of Measurement and 38 Direction of Vibration

5.5.3 Seat Versus Floorboard Measurements 39

5.5.4 Significance of Frequency Range Considered 39

5.6 Summary 40

RIDE EVALUATION EQUATIONS

6.1 Proposed Comfort Equation 41

6.2 Discomfort Equation 42

CONCLUSIONS AND RECOMMENDATIONS

REFERENCES $\quad 47$

$\begin{array}{ll}\text { APPENDIX A } & 48\end{array}$

A. 1 Introduction 48

A.2 Detrending 49

A. 3 Power Spectrum Calculations 50

A.4 Data Averaging 50

APPENDIX B 51

Subjective Rating Form $\quad 51$ 
Page

APPENDIX $C$

Spectral Density Plots

APPENDIX D

Weighted Indices

82

APPENDIX E

E.1 Scattergrams

E.2 Correlation Coefficients and Ride Index Equation Parameters for Various Weighting Functions 
Figure 2.1: NASA Accelerometer Package

Figure 2.2: Endevco Accelerometer Package, Signal Conditioner and Power Supply

Figure 2.3: TEAC R200 DR/FM Data Recorder 9

Figure 5.1: Frequency Weighting Functions $\quad 27$

Figure 5.2: Absorbed Power Weighting Values 29

Figure 5.3: Correlation of Weighted Indices for Maverick Seat Vibrations with Mean Personal Ratings

Figure 5.4: Correlation of Weighted Indices for Maverick Floor Vibrations with Mean Personal Ratings

Figure 5.5: Correlation of Weighted Indices for Buick Floor Vibrations with Mean Personal Ratings

Figure 5.6: Correlation of Weighted Indices for Combined Buick and Maverick Floorboard Vibrations with Mean Personal Ratings

Figure 6.1: Least Squares Curve Fit to Magnitude RMS Accelerations Within the 0 to 40 Hertz Band vs. Mean Personal Ratings

\section{LIST OF TABLES}

$\begin{array}{lll}\text { Table 4.1: } & \text { UTACV Graphical Analysis } & 18\end{array}$

Table 4.2: ISO Graphical Analys is $\quad 19$

Table 5.1: Frequency Weighting Functions (frequency in hertz) 25

Table 5.2: Absorbed Power Weighting Functions 30

Table 5.3: Correlation Coefficients 33 


\section{NOMENCLATURE}

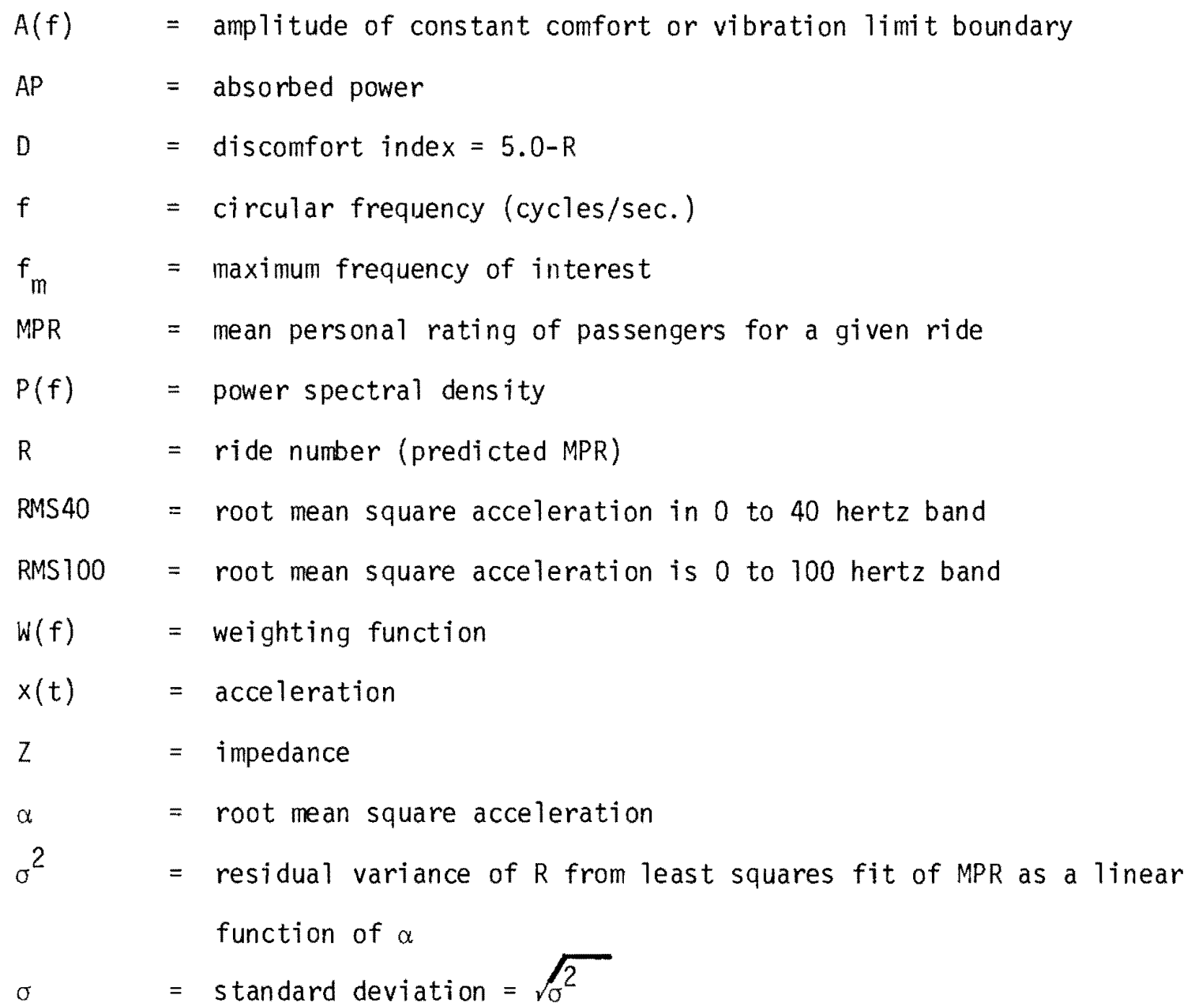

Subscript

w

= weighted value 


\section{INTRODUCTION}

In transportation vehicles, an important part of the passenger's environment is the vibration to which he is subjected. Information concerning the response of passengers to a vehicle vibration environment has become increasingly important for use in the development of new transportation systems. While underdesign of a new system with regard to allowed vibration levels can cause it to be uncomfortable and hence unacceptable to the traveling public, overdesign (where vibrations that would normaliy not affect the passenger's perception of comfort significantly are eliminated) can result in excessive system cost. Indeed, in many cases system cost is very strongly related to the ride quality criteria that may be imposed upon the designer. This study is an attempt to relate passengers' perceptions to measured riding vibrations in order to evaluate some design criteria presently in use and to develop better criteria for vehicle ride comfort design.

The automobile was chosen as the vehicle upon which to base this study for a variety of reasons. Firstiy, it represents a riding environment with which most people are familiar. This tends to make it a common basis upon which to begin when rating ride quality, and will allow extrapolation to new environments based upon experience common to the majority of passengers. Secondly, the automobile is relatively convenient for use in this type of field study. It is relatively easy to control the general passenger environment, the roughness of the roadway, etc. Thirdly, previous work at The University of Texas Center for Highway Research, where similar types of field studies have been used to evaluate pavement "serviceability" or roughness $[1]^{*}$, provides useful background information and experience for this type of study.

* Numbers in [ ] refer to references in Bibliography. 


\subsection{Existing Criteria}

A number of ride quality "criteria" are presently in existence. Notable among these are the International Standards Organization (ISO) "A Guide to the Evaluation of Human Exposure to Whole Body Vibration" [2], Lee and Pradko's "Absorbed Power" [3], The Urban Tracked Air Cushion Vehicle (UTACV) Specification [4], and acceleration limits as a function of frequency given by Janeway [5] and Dieckmann [6]. A review of the literature discussing the origin of these criteria as well as other work in the area is included in [7]. In general, the limits of Janeway and Dieckmann are rms acceleration limits for pure sinusoidal vibrations. The concept proposed is that if the rms amplitude of each sinusoidal component contained within the ride does not exceed the proposed limits, the ride should be comfortable. The difficulties with using these criteria are twofold. Firstly, riding vibrations are never pure sinusoids and even though basic components can be isolated by bandpass filtering the amplitude is strongly dependent upon the filter characteristics. As one manufacturer put it, "all we had to do was to reduce the bandwidth until we met the criteria." The second problem associated with this type of criteria is that it assumes no interaction between components of different frequencies. That is to say it is not intuitively satisfying to assume that a ride with two basic vibration components at different frequencies, both of which are just below the limits, is a better ride than another with only one vibration component which is a little above the limit. (Note that in the latter case the total rms vibration level could far exceed the first case.)

The ISO Standard is of essentially the same form as the limits of Janeway and Dieckmann, except it specifies the bandwidth over which the rms at any given "center frequency" should be calculated as one-third octave. This helps somewhat, but the difficulty of differentiating between multiple component vibrations remains. 
The UTACV Specification is a boundary below which the power spectral density (PSD) of the ride accelerations must be at all frequencies. This specification also avoids the bandwidth problem (if care is used in calculation of the PSD), but again retains the problem of multiple components or regions where the PSD just meets the specification vs. one component which exceeds only at one point with all other points well below the boundary.

The "absorbed power" concept of Lee and Pradko differs somewhat in approach from the other criteria discussed in that it relates the quality of the ride to a single number, i.e. the average power absorbed by the passenger due to the riding vibrations during the ride. The average absorbed power is computed as a weighted integral of the acceleration spectral density, where the weighting function is the squared magnitude of the mechanical impedance of the human at the boundary between the human and the medium imposing the motion where the acceleration is measured. Lee and Pradko define impedances for various body locations for an "average man". This concept is somewhat appealing from a designer's point of view since it reduces all ride variables to a single scaler number which is "representative" of the ride quality. The primary difficulty here is that the criterion has not been suitably verified in a realistic ride environment.

Closely related to the approach of Lee and Pradko is the approach proposed by Butkunas [8], using a weighted rms acceleration as a one-number ride index. Butkunas indicates that any one of the proposed comfort limits (such as those mentioned above) represents human sensitivity to vibration, and as such, can be used to develop weighting functions or "transfer functions". One difference between this approach and that of Lee and Pradko is that the weighting function is defined by a generalized transfer function representing human perception of ride, whether that perception relates to the mechanical impedance or not, whereas the absorbed power method uses only the mechanical impedance as a weighting 
function. Another difference lies in the fact that even when the mechanical impedance is the weighting function for both approaches, the weighted rms acceleration is proportional to the square root of the absorbed power rather than proportional to the absorbed power. Thus the two approaches assume a different functional relationship between amplitude of ride vibrations and human sensitivity.

\subsection{Objectives and Scope of Study}

The objective of this study is to examine each of the ride quality evaluation methods presented above on the basis of a field experiment. The field experiment used two different automobiles (a 1974 Buick Century Luxus and a 1975 Ford Maverick), 78 different passengers, and 18 different roadway sections. Both objective measurements of the riding vibrations and subjective measures of the passengers' perceptions of each ride were taken.

A preliminary examination of the data consists of a study of the ability of the UTACV Specification and the ISO Standard to indicate or predict the passengers' perceptions. The larger, remaining part of the study examines the use of each of the above boundary type criteria (ISO, UTACV, Janeway, Dieckmann) as frequency weighting functions to calculate frequency weighted rms acceleration ride indices as proposed by Butkunas. In addition, absorbed power ride indices are also calculated. The coefficient of correlation between the ride indices and the mean personal (subjective) ratings of the passengers in each case is then examined. Relative effects of each weighting function or method are compared.

The measurement of riding vibrations, signal processing, filtering, and spectral density calculations are described in Chapter 2. In Chapter 3 the psychometric experiment used to measure the passenger's "mean personal rating" 
or subjective evaluation of the ride is discussed. The ISO Standard and the UTACV Specification are examined in detail relative to the experimental data in Chapter 4. In Chapter 5, the various frequency weighting functions are described and compared on the basis of their ability to produce a weighted value which correlates with the mean personal (subjective) ratings of the passengers. In Chapter 6 , a ride quality criterion is presented based upon the results of Chapter 5 . Conclusions and recommendations are summarized in Chapter 7. 


\section{MEASUREMENT AND PROCESSING OF ACCELERATION DATA}

\subsection{Measurement of Acceleration Data}

The acceleration data used in this experiment included vertical and lateral accelerations on the floorboard of a 1974 Buick Century Luxus and a 1975 Ford Maverick, and vertical and lateral accelerations of a 12 inch circular disk of $1 / 4$ inch plywood placed between the seat and a $1551 \mathrm{~b}$. passenger who was sitting on the disk. The accelerations were measured while the vehicles were driven at $50 \mathrm{mph}$, approximately 2 foot left of center of the road, over highway test sections designated by the Texas State Department of Highways and Public Transportation. These sections are approximately $1250 \mathrm{ft}$. long and contain approximately stationary profiles (no curves or significant grades) throughout each individual test section. Each section has been given a serviceability index (S.I.) by the Texas State Department of Highways and Public Transportation which is an indication of its roughness on a six point scale $(0=$ very rough, $5=$ very smooth $)$. The sections used ranged in S.I. ratings from 1.9 to 4.3 , including a varity of roughnesses from some fairly rough farm to market road sections to very smooth interstate highway.

To measure the accelerations on the floorboards, a three axis accelerometer package loaned to The University of Texas by the NASA Langley Research Center (Figure 2.1) was used. The package is self-contained, including its own batteries and signal conditioning equipment. The frequency response of the accelerometer package supplied by NASA is about $3 \mathrm{db}$. down at 100 hertz, effectively low pass filtering the data measured by it. The metal box containing the accelerometer package has 3 metal "spike" feet which penetrate the carpet and contact the metal floor pan. To avoid bouncing of the package on the floorboard (should it otherwise occur), the package was lodged securely under the rear of the front seat on the passenger (right) side of the car. 


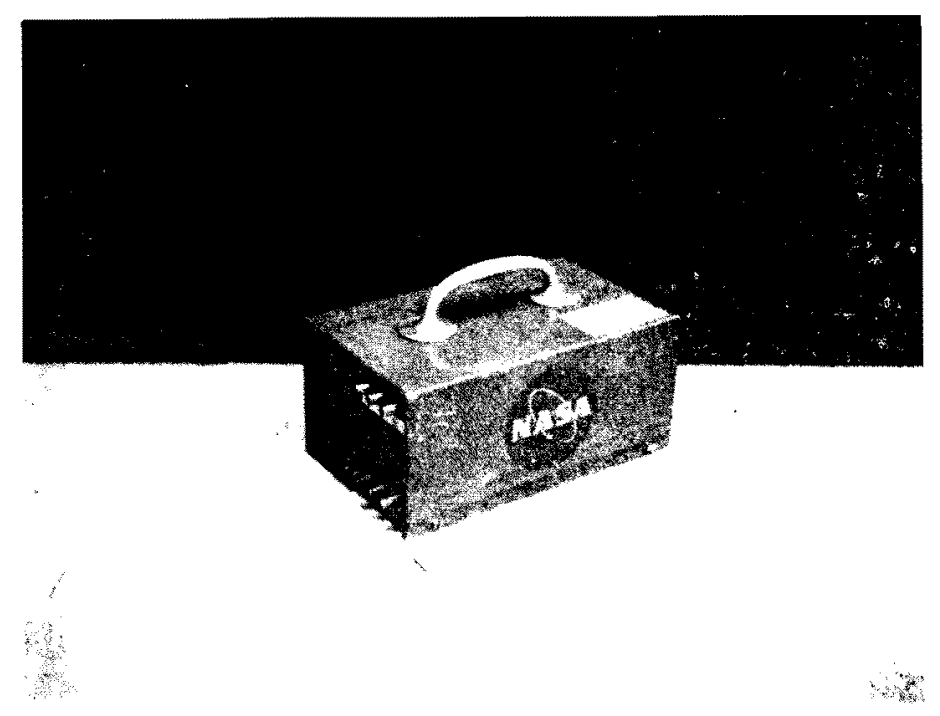

Figure 2.1. NASA Accelerometer Package

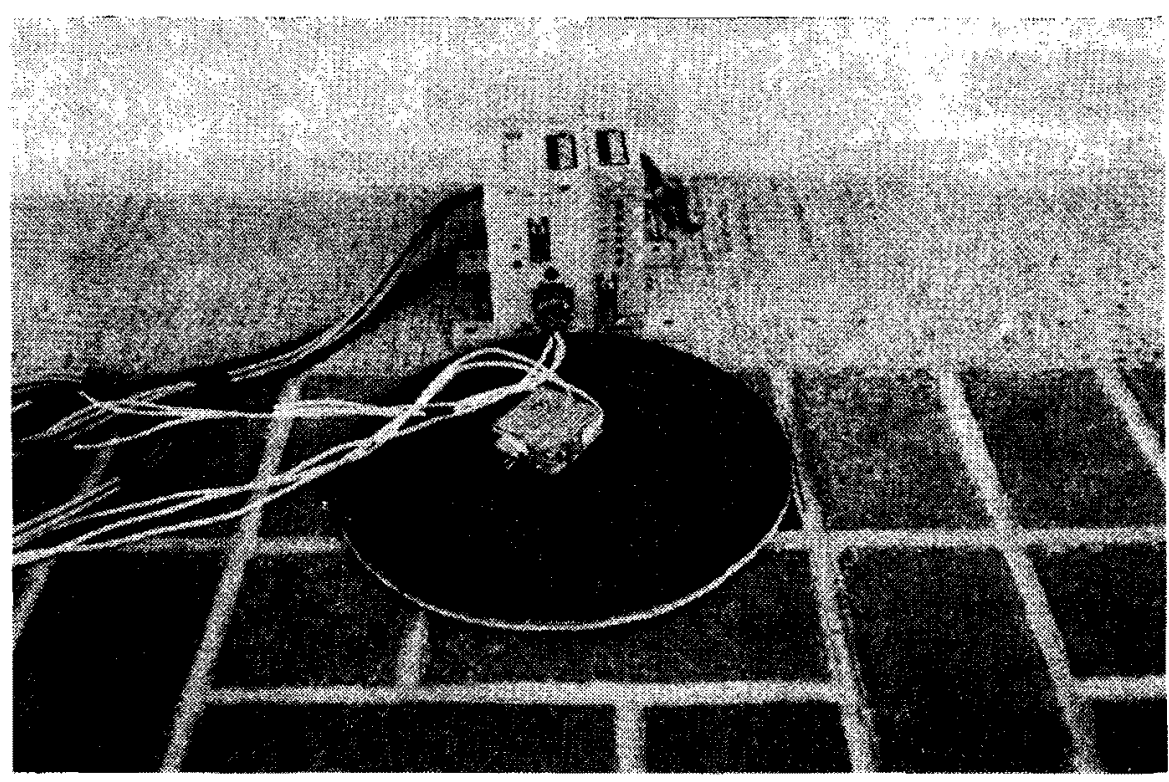

Figure 2.2. Endevco Accelerometer Package, Signal Conditioner and Power Supply 
The seat accelerations were measured using a smaller accelerometer package consisting of a triaxial arrangement of Endevco mode1 2265-20 accelerometers, an Endevco model 7441 signal conditioning module, and an appropriate power supply (Figure 2.2). The small triaxial accelerometer package was mounted on a 12-inch-diameter circular disc of 1/4-inch plywood. The disc was placed underneath the passenger on the front passenger seat.

For each test section and car combination, vertical and lateral acceleration signals were recorded on the first two channels of a TEAC R200 DR/FM Data Recorder (see Figure 2.3) while verbal commentary was recorded on the fourth channel. Since for the Ford Maverick both seat and floorboard vibrations were measured, two separate runs over each test section were required, one for the floorboard and one for the seat. During the measurement process, the car contained 3 passengers (including the driver) and the tape recorder on the rear seat on the right side of the car, making the load distribution somewhat similar to the load distribution of 4 passengers (including the driver) during the subjective ride evaluation tests described in the next chapter.

\subsection{Digitizing of Data}

After the acceleration measurements were recorded, the analog signals were digitized for processing by digital computer. During the course of the experiment, two different facilities were used for the digitizing process. For the Buick vibrations a Hewlett-Packard 2115 Computer with A/D converter which is owned by the Texas State Department of Highways and Public Transportation was used to sample and digitize the signals at a sampling rate of 434 samples per second. The Maverick vibrations were digitized at the Hybrid Computer Laboratory at The University of Texas. The sampling rate for this data was also 434 samples per second, and the samples were read into an SDS 930 computer. During the digitizing of the Maverick data, a Hewlett-Packard 4509A low pass filter 


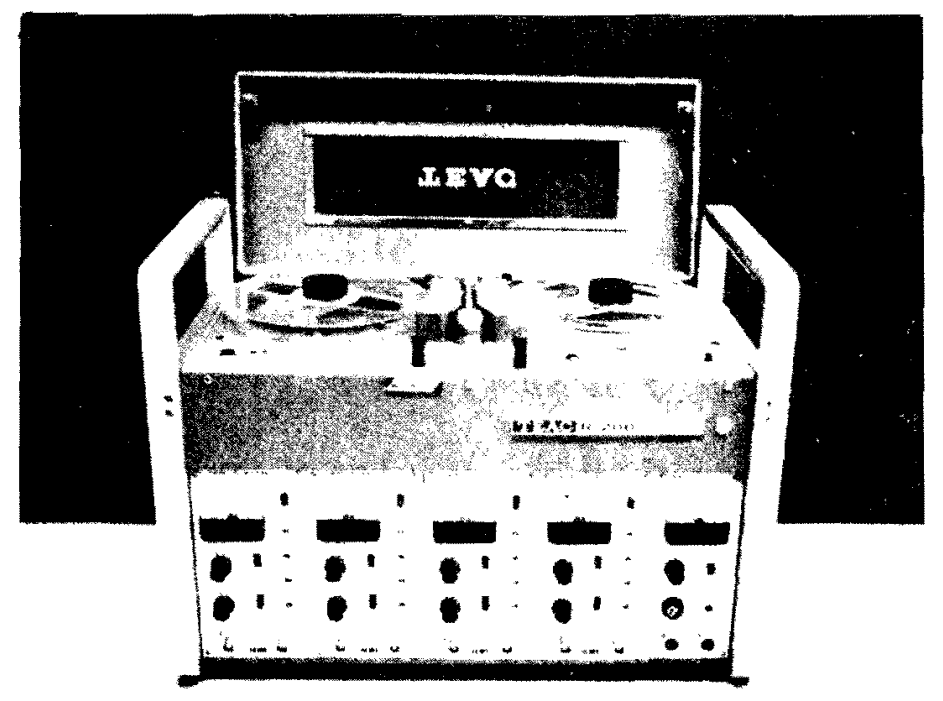

Figure 2.3. TEAC R200 DR/FM Data Recorder 
with 100 hertz cutoff frequency was used ahead of the sampler to avoid aliasing problems. For the previously digitized Buick Data this was not necessary because the NASA - Langley accelerometer package itself has a frequency response which rolls off at about 100 hertz. For both of these processes, the digitized data were written on magnetic tape (7 track) at 556 bpi. These magnetic tapes were then read into The University of Texas CDC $6400 / 6600$ digital computer for signal processing.

\subsection{Spectral Density Calculations}

A11 of the processing of the data described in this report requires calculation of the spectral density, or more commonly but less properly the "power spectral density" (PSD) of each of the acceleration profiles. The spectral density, defined as the Fourier transform of the autocorrelation function, is a measure of the average squared magnitude of the signal content per unit bandwidth as a function of frequency. Efficient calculation of the spectral density of a finite data sequence such as the test section acceleration profiles described herein is accomplished using the Fast Fourier Transform (FFT) algorithm. The procedure for these calculations, including windowing and data averaging, is described in detail in Appendix A.

\subsection{Weighted RMS Acceleration "Ride Index" Calculations}

From the power spectral density of a given signal, the mean square amplitude of the signal can be calculated as the integral over all frequencies of the PSD, or

$$
\overline{x^{2}(t)}=\int_{0}^{\infty} P(f) d f
$$

where $x(t)$ is the signal as a function of time $t$, and $P(f)$ is the PSD as a function of circular frequency $f$. For a sampled signal, frequency content is limited to the Nyquist folding frequency (one-half the sampling frequency), or our interest 
may otherwise be limited to signal content below some given frequency $f_{m}$ so we may write

$$
\overline{x^{2}(t)}=\int_{0}^{f} P(f) d f
$$

where all components above $f_{m}$ are either assumed to be negligible or not to be of interest. The root mean square (rms) value is then the square root of $\overline{x^{2}(t)}$ or

$$
\alpha=\sqrt{x^{2}(t)}=\sqrt{\int_{0}^{m} P(f) d f}
$$

Since it is reasonable to assume that passengers are more sensitive to acceleration "power" at some frequencies than others, a weighted mean square acceleration level can be defined as

$$
\overline{x_{W}{ }^{2}}=\int_{0}^{f_{m}} W(f) P(f) d f
$$

where $\overline{x^{2}}$ represents the weighted mean square value and $W(f)$ is a weighting function which reflects the desired weighting to reflect passenger sensitivity. Actual weighting functions used in this study are described subsequently and are based upon various constant comfort contours. Calculation of the weighted mean square value $\overline{x_{W}^{2}}$, given the functions $W(f)$ and $P(f)$ as sampled data sequences, is accomplished through numerical integration using Simpson's rule. The weighted root mean square ride index is then calculated as simply the square root of $\overline{x_{W}^{2}}$ :

$$
\alpha_{W}=\sqrt{\overline{x_{W}^{2}}}=\sqrt{\int_{0}^{f_{m}} w(f) P(f) d f}
$$

The various weighting functions $W(f)$ used and compared in this study are described in detail in Chapter 4, along with the curves from which they were obtained. 


\section{PSYCHOMETRIC EXPERIMENTS FOR RIDE EVALUATION}

To determine the subjective rating of a variety of passengers in different riding environments, a two-part psychometric experiment was performed. The first part of the experiment, conducted during the spring of 1974 used a 1974 Buick Century Luxus automobile as the test vehicle. After examination of the results of the first part, the second part was done in December, 1974, using a 1975 Ford Maverick as the test vehicle.

\subsection{Buick Rating Sessions}

3.1.1 General Each of 24 subjects was driven in the same 1974 Buick Century Luxus over six road sections. Of these sections, two were rough, two were medium, and two were smooth, based upon their SI as described below. Many background variables were considered in order to reveal any biasing of the subject due to his daily environment. Background variables such as personality measures, age, and type of vehicle normally driven were included in an attempt to relate these variables to the subject's rating ability.

3.1.2 Routes The total of eleven roadway test sections used in this part of the experiment were divided into four routes. Each of these routes contained one each of the rough, medium and smooth categories of road sections. One test section (Section 5) was used on two routes. These road sections were obtained from the Texas State Department of Highways and Public Transportation, which measures and studies these sections periodically four times a year. Each section is given a Serviceability Index (SI) value which is computed from measurements of the road surface. These measurements are taken with a General Motors Surface Dynamics Profilometer (for more information on profilometer see [9]), which measures the profile of the road in the wheel tracks of the vehicle. The SI values may range from 0 to 5 ( 5 being smoothest and 0 being roughest); however, the sections available for use on this experiment ranged from 2.3 to 4.3. 
The sections were grouped according to their S.I. values as follows:

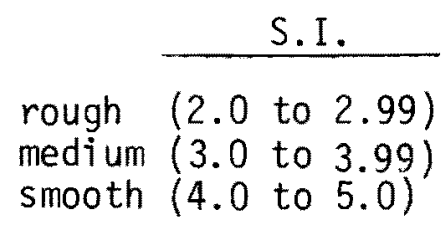

3.1.3 Design Each subject was driven over two routes and made six ratings of ride quality. To avoid the possibility of bias in the ratings of ride quality the driving sequence of sections within routes was varied systematically. This was accomplished by reversing the order of sections within routes $(1,2,3$ to $3,2,1)$ and varying the rough, medium, and smooth order from one route to another in order that approximately one third of the routes started with smooth sections, another one third started with medium sections, and so on. Similarily, the second and third sections were equally often smooth, medium, or rough. This accounted for any variance in the rating ability of the subjects which could have been affected by practice, boredom, or fatigue acquired during the rating session.

3.1.4 Subjects Twenty-four subjects served in this experiment. Half of these were driven over two of the routes and the other half over the other two routes. Subjects were obtained from introductory psychology classes and served in the experiment to fulfill a laboratory requirement.

3.1.5 Procedure Groups of three subjects were used in each session of the experiment. The group was assembled at The University of Texas, where personality tests and background questionnaires were completed. Next, they were seated in the automobile, two in the back seat and one in the front seat, and driven to the appropriate test sections. The same driver drove the car for all subjects. Care was taken by the driver to maintain the same conditions throughout the rating sessions, which included emphasizing the importance of the experiment. The subjects were driven over the test sections at 50 m.p.h. approximately 2 feet to the 
right of the highway center stripe and then asked to evaluate the ride using the rating questionnaires (See Appendix B). Ratings were defined to range from 1 ("the worst ride I can think of") to 5 ("the best ride I can think of").

3.1.6 Results The analysis of the data yielded several items of significance. Individual ratings were found to have significant agreement with the Mean Personal Ratings (M.P.R.) as well as (S.I.) values previously mentioned. The correlation between the S.I. values and M.P.R. values was .92. Also, it was found that personality and background variables had little effect upon the subjects' ratings. The main significance of these findings from the standpoint of this study is the fact that the personal ratings of the ride are repeatable and valid and can therefore be compared to the study of the measured vehicle vibrations.

\subsection{Maverick Rating Session (Fal1 1975)}

\subsubsection{General Each of 54 subjects was driven in a 1975 Ford Maverick over} nine sections. Of these sections, three were rough, three medium, and three smooth. Unlike in the Buick study, all subjects rated all of the road sections; however, driving order and controlled conditions varied according to the design of the experiment. Due to the findings of the previous experiment, background differences were not considered. Instead, subjects in different rating sessions were exposed to different audio and visual stimuli during the runs over the sections.

3.2.2 Design Nine road sections were used in this study, with each road section being rated by each of 54 passengers. The driving route was broken up into three groups, each of which contained a rough, medium, and smooth section with regard to S.I. values as in the previous study using the Buick. The order of rough, medium, and smooth sections in each of the groups was different. In addition, the driving sequence was varied by switching the order of the groups systematically to avoid the possibility of bias in the ratings. Each subject was driven over the nine sections of the route and made a rating of each section immediately after riding over the section. 
3.2.3 Sensory Modes While travelling over the road sections the subjects were put in three different sensory modes to judge the road sections. These are referred to as the "deleted", "added", and "control" modes in both audio and visual senses. The "deleted" mode used a blindfold for visual effect and earplugs and headset for audio. For "added" stimuli, the subjects were asked to search for letter targets on a sheet of paper for the visual mode and from a recorded tape for the audio mode. The "control" mode required just sitting still. Audio and visual modes were not mixed between the passengers in each group.

3.2.4 Subjects Fifty-four subjects served in this experiment and, as in the prior study, they were obtained from introductory psychology classes and served in the experiment to fulfill a laboratory requirement.

3.2.5 Procedure Groups of three subjects were used in each session of the experiment. A group was assembled at The University of Texas, and instructions for the respective tasks were given and forms were completed. Next, they were seated in the automobile, two in the back seat and one in the front seat, and driven to the appropriate starting point. For all subjects, a 1975 Ford Maverick was used as the test vehicle and the driver was the same. Care was taken by the driver to maintain the same conditions throughout the rating sessions, except for the planned differences in the audio and visual inputs. Each group had two audio or visual tasks to perform for six roadway sections and control conditions for the other three sections. Prior to reaching a roadway section, the subjects were allowed to prepare for the section as they had previously been instructed. They were then driven over the test sections at 50 m.p.h., approximately two feet right of the highway center stripe, and asked to evaluate the ride using the questionnaires (See Appendix $B$ ). Within a given group al1 subjects were in the same sensory mode at a given time. As in the previous 
experiment, ratings were defined over the range from 1 ("the worst ride I can think of"), to 5 ("the best ride I can think of").

3.2.6 Results The analysis of the data yielded several items of significance. The main point, however, was that Mean Personal Rating (MPR) again had a reiatively high correlation with the SI value $(=.87)$. This is slightly lower than the previous test with the Buick $(=.92)$; however, this is understandable since the SI equations were derived from a regressive fit of profile data with data from similar rating sessions using a full-sized sedan similar to the Buick. Since the Maverick was smaller, a less comfortable ride was possible for certain sections. 


\section{COMPARISON OF PASSENGER RATINGS WITH ISO AND UTACV SPECIFICATIONS}

The ISO Standard and the UTACV Specification describe boundaries which are interpreted as defining the acceptability of given riding vibrations. It is therefore instructive to examine by graphical analysis the vibration spectra of the riding vibrations measured in this study to determine to what degree each of these criteria relates to the mean personal subjective rating of the passengers for each ride.

\subsection{Comparison with UTACV Boundaries}

To compare each of the rides with the UTACV Specifications, the spectral density of the vertical and lateral vibrations measured at the floorboard of the Buick and at the floorboard and seat of the Maverick were plotted against the specification. The corresponding plots are shown in Appendix C. By inspection of these plots each section was classified as rough (R), medium (M), or smooth (S) with regard to each of its characteristic spectra (vertical, lateral, floor, seat). A given spectrum was characterized as smooth if it was below the boundary at all frequencies, medium if only small peaks were above the boundary, and rough if large multiple peaks were above the boundary. Admittedly, a great deal of subjectivity was required in these categorizations. The results of this graphical analysis are presented in order of decreasing MPR (decreasing passenger comfort rating) in Table 4.1 .

As can be seen from Table 4.1 , none of the sections was rated smooth with regard to all spectra. In other words all the automobile rides measured had spectra which exceeded the UTACV boundaries at some frequencies, indicating the boundary may be somewhat too conservative in what it allows. Also notable is the fact that even though rides with lower MPR's tended to have "rougher" spectra, there is some overlap. For instance, Buick section 3, with a MPR of 3.83, has R vertical and M lateral spectra whereas Buick sections 1 and 8 , both having a MPR of 3.50 , have $M$ vertical and lateral spectra. Vertical and lateral spectra seem to be rated 
Table 4.1 UTACV Graphical Analysis

\begin{tabular}{|c|c|c|c|c|c|c|}
\hline \multicolumn{7}{|c|}{ Maverick (Seat and Floor) } \\
\hline \multirow{2}{*}{ Section } & \multicolumn{2}{|c|}{ Floor } & \multicolumn{2}{|c|}{ Seat } & \multirow{2}{*}{ MPR } & \multirow{2}{*}{ SI } \\
\hline & vertical & Lateral & vertical & & & \\
\hline 9 & $M$ & M & $S$ & M & 4.09 & 4.2 \\
\hline 10 & $M$ & M & $S$ & $M$ & 4.07 & 4.3 \\
\hline 14 & $M$ & M & M & $M$ & 3.85 & 3.4 \\
\hline 36 & $M$ & M & $S$ & $M$ & 3.80 & 4.2 \\
\hline 40 & $M$ & $R$ & $S$ & $\mathrm{R}$ & 3.39 & 3.5 \\
\hline 15 & $R$ & R & M & $\mathbf{R}$ & 2.72 & 3.6 \\
\hline 39 & $\mathrm{R}$ & $R$ & M & $R$ & 2.67 & 1.9 \\
\hline 35 & $\mathbf{R}$ & M & M & $\mathrm{R}$ & 2.54 & 2.0 \\
\hline 38 & $R$ & $R$ & $\mathrm{R}$ & $R$ & 2.20 & 2.0 \\
\hline \multicolumn{7}{|c|}{$\begin{array}{l}S=\text { smooth } \\
M=\text { medium } \\
R=\text { rough }\end{array}$} \\
\hline \multicolumn{7}{|c|}{ Buick (Floor Only) } \\
\hline & Section & Vertical & Transverse & MPR & SI & \\
\hline & 5 & $M$ & M & 4.42 & 4.0 & \\
\hline & 7 & $M$ & M & 4.25 & 4.3 & \\
\hline & 9 & $M$ & $M$ & 4.17 & 3.9 & \\
\hline & 3 & $R$. & $M$ & 3.83 & 3.4 & \\
\hline . & 1 & $M$ & M & 3.50 & 3.5 & \\
\hline & 8 & $M$ & $M$ & 3.50 & 3.4 & \\
\hline & 6 & $R$ & M & 3.00 & 2.8 & \\
\hline & 41 & $R$ & $M$ & 3.00 & 2.6 & \\
\hline & 2 & $R$ & $M$ & 2.33 & 2.7 & \\
\hline & 39 & $R$ & $R$ & 2.33 & 2.3 & \\
\hline & 37 & $R$ & M & 2.23 & 3.0 & \\
\hline
\end{tabular}


Table 4.2 ISO Graphical Analysis

Maverick (Seat and Floor)

\begin{tabular}{|c|c|c|c|c|c|c|}
\hline \multirow{2}{*}{ Section } & \multicolumn{2}{|c|}{ Floor } & \multicolumn{2}{|c|}{ Seat } & \multirow{2}{*}{ MPR } & \multirow{2}{*}{ SI } \\
\hline & Vertical & Transverse & Vertical & Transverse & & \\
\hline 9 & $S$ & $S$ & $S$ & $S$ & 4.09 & 4.2 \\
\hline 10 & $S$ & $S$ & $S$ & $S$ & 4.07 & 4.3 \\
\hline 14 & $S$ & $S$ & $S$ & $S$ & 3.85 & 3.4 \\
\hline 36 & $S$ & $S$ & $S$ & $s$ & 3.80 & 4.2 \\
\hline 40 & M & $S$ & $S$ & $S$ & 3.39 & 3.5 \\
\hline 15 & $R$ & $S$ & $S$ & $S$ & 2.72 & 3.6 \\
\hline 39 & $\mathrm{R}$ & M & $\mathrm{R}$ & M & 2.67 & 1.9 \\
\hline 35 & $\mathrm{R}$ & $S$ & $\mathrm{R}$ & $\mathrm{R}$ & 2.54 & 2.0 \\
\hline 38 & $\mathrm{R}$ & $R$ & $\mathrm{R}$ & $\mathrm{R}$ & 2.20 & 2.0 \\
\hline \multicolumn{2}{|c|}{$\begin{array}{l}S=\text { smooth } \\
M=\text { medium } \\
R=\text { rough }\end{array}$} & \multicolumn{3}{|c|}{ Buick (Floor Only) } & & \\
\hline & Section & Vertical & Transverse & MPR & SI & \\
\hline & 5 & $S$ & $S$ & 4.42 & 4.0 & \\
\hline & 7 & $S$ & $S$ & 4.25 & 4.3 & \\
\hline & 9 & $S$ & $S$ & 4.17 & 3.9 & \\
\hline & 3 & $M$ & $S$ & 3.83 & 3.4 & \\
\hline & 1 & $S$ & $S$ & 3.50 & 3.5 & \\
\hline & 8 & M & $S$ & 3.50 & 3.4 & \\
\hline & 6 & $R$ & $S$ & 3.00 & 2.8 & \\
\hline & 41 & $R$ & $S$ & 3.00 & 2.6 & \\
\hline & 2 & $R$ & $S$ & 2.33 & 2.7 & \\
\hline & 39 & $\mathrm{R}$ & $S$ & 2.33 & 2.3 & \\
\hline & 37 & $R$ & $S$ & 2.23 & 3.0 & \\
\hline
\end{tabular}


about equally in "roughness", indicating neither to be dominant in determining the quality of the ride. All rides with MPR less than or equal to 3.39 had an $R$ rating of the spectrum for either the vertical or lateral directions or both.

\subsection{Comparison with ISO Boundaries}

An analysis similar to that above was also performed using the ISO Standard. In this case the rms acceleration within each one-third octave band from 1 hertz to 80 hertz was plotted versus the ISO 8 hour and 1 hour boundaries for each spectra of interest. These plots are also found in Appendix $C$. Also plotted with the power spectral density plots used in the UTACV analysis above are "equivalent" ISO boundaries which represent the magnitude that the average value of the PSD within the one-third octave band centered at a given frequency cannot exceed in order for the RMS value within the band to be below the original ISO boundary. (For development see [10].) As with the UTACV analysis, each spectrum was categorized as smooth, medium, or rough. In this case, all categorizations were defined relative to the 8 hour boundary, where smooth was the rms level with all bands being below the boundary, medium was the rms level with only one band being on or above the boundary, and rough was the rms level with multiple bands being on or above the boundary. The results are shown in Table 4.2.

In this case, all spectra with mean personal ratings greater than or equal to 3.85 were rated as smooth. All sections except sections 39 and 38 in the Maverick were rated smooth with regard to floorboard lateral vibrations, which would tend to indicate that either the vertical vibrations are more important in determining the ride quality for these cases or the lateral boundary is less restrictive than the vertical relative to a given ride comfort level. Again there is some overlap between roughness ratings (for example, the vertical spectra for section 3 in the Buick is rated M, with a MPR of 3.83 , while section 1 is rated 
S, with an MPR of 3.50 ), but there is a very definite trend for "rougher" ratings with decreased MPR. All rides with a MPR of 3.00 or less were rated $R$ in at least one of the spectra measured on the floorboard, whereas only rides with a MPR of 2.67 or less were so rated by spectra measured at the seat. 


\section{FREQUENCY WEIGHTED RIDE INDICES AND THEIR CORRELATION WITH MEAN PERSONAL RATINGS}

\subsection{Weighting Functions}

Since the boundary-type criteria proposed by Janeway, Dieckmann, ISO, and the UTACV specification represent in some sense human sensitivity to vibration as a function of frequency, each can be used to develop a "transfer function" relating human sensitivity to the riding vibrations. Stated in other terms, each can be used to develop a weighting function which can be used to weight the vibration spectra according to each criterion's definition of the sensitivity of man to vibrations at given frequencies.

If the "boundary" specifies, for instance, the maximum level which should not be exceeded by the magnitude of a sinusoidal vibration, then one might wish to use the value of the magnitude of the sinusoidal vibration divided by the value of the boundary at the corresponding frequency as a ride ratio or index of the ride by which various sinusoidal rides at different frequencies could be compared. Carrying this a little further, rides consisting of multiple sinusoids could be compared by adding the ride ratios for all of the individual components of the ride to get an overall ride index. In the limit, for the spectrum of a nonperiodic vibration, one would weigh the amplitude spectrum of the vibration by the inverse of the value of the boundary, and integrate over the spectrum of interest. To apply this general concept to the power spectral density, since the power spectral density of a signal is related to the amplitude squared, one might let the weighting function be the amplitude of the square of the bourdary and the integral be the ride index squared. The weighted index is then

$$
\alpha_{w}=\sqrt{\int_{0}^{f^{m}}(f) P(f) d f}
$$


where

$$
W(F)=\frac{1 / A^{2}(f)}{\int_{0}^{f} m 1 / A^{2}(f) d f}
$$

is the weighting function. In the equations above, 0 and $f_{m}$ are the endpoints of the frequency range of interest and $A(f)$ is the amplitude of the boundary when using the Janeway, Dieckmann, and ISO boundaries while $A^{2}(f)$ is the magnitude of the boundary when using the UTACV boundary (since the UTACV boundary is a boundary imposed upon the power spectral density rather than vibration amplitude and therefore represents sensitivity to the square of the amplitude spectrum). The denominator in (5.2) is included arbitrarily to normalize the weighting function such that a vibration with constant spectrum (white noise) over the frequency range of interest would have a weighted index (or weighted rms) equal to its rms value. This normalization has no effect upon the correlation (or lack of it) between the weighted indices and the mean personal ratings in this study as long as only one weighting function is used to calculate all indices in a given correlation. It does affect the absolute magnitude of the weighted index, but, since it in effect multiplies all weighted indices calculated using the particular weighting function by the same scale factor, it does not affect the linear correlations. It is included here to keep the weighted indices within the same order of magnitude. One must remember, however, that the exact magnitude of the weighted indices cannot be compared between different weighting functions. In addition, since vertical and lateral weighting functions are generally different, relative magnitudes of vertical and lateral indices could be changed by a change in the normalization procedure. Therefore the indices in this study referred to as "magnitude" indices, defined as the square root of the sum of the squares of the vertical and lateral indices (described in Section 5.4), could be changed by 
changing the normalization procedure which effectively weights the relative effects of vertical and lateral motions. No attempt was made in this study to determine the "optimal" relative weighting between vertical and lateral components.

The actual equations used for the boundaries and weighting functions for each of the cases considered are listed in Table 5.1. For comparison purposes, the shapes of the weighting functions are plotted in Figure 5.1. The absolute magnitude for each of the weighting functions shown is arbitrary and was adjusted on the plot so the shape of each section could be shown throughout most of the spectrum of interest.* The magnitudes on the ordinates of the plots are given only to indicate the order of magnitude change in weighting from one frequency to another for any given weighting function. It is also noted that since the ISO and Janeway boundaries are not defined below 1 hertz, their values at 1 hertz were extrapolated to zero. In addition, the Janeway boundary was extrapolated above 60 hertz at constant slope and the ISO boundary was extrapolated above 80 hertz at constant slope. The UTACV boundary, on the other hand, was only used to weight spectra up to 50 hertz, all spectra above being neglected (effectively zero weighting above 50 hertz). In al1 cases, spectra above 100 hertz were neglected. Since Dieckmann and Janeway proposed only vertical limits, corresponding lateral boundaries do not exist as they do for the ISO and UTACV boundaries. The weighted indices based upon each of these weighting schemes were calculated and are tabulated in Appendix D. In the cases where both vertical and lateral weighted indices were calculated, a weighted magnitude was also calculated, defined as the square root of the sum of the squares of the vertical and lateral weighted indices.

\footnotetext{
*It would be correct to say for instance from Figure 5.1 that the Dieckmann weighting curve weights low frequencies more heavily relative to high frequencies in a spectrum than does the Janeway weighting scheme. It would not be correct to say simply that the Dieckmann weighting curve weights low frequencies more heavily than the Janeway weighting curve.
} 
Table 5.1. Frequency Weighting Functions (frequency in hertz)

Janeway (vertical only):

$$
\begin{array}{rl}
0<f \leq 1 & W(f)=(25.00) / N F \\
1<f \leq 6 & W(f)=\left(25.00 f^{2}\right) / N F \\
6<f \leq 20 & W(f)=(900.00) / N F \\
20<f \leq 100 & W(f)=\left(444,631 / f^{2}\right) / N F
\end{array}
$$

Normalizing factor $(N F)=28,805$

Dieckmann (vertical only):

$$
\begin{array}{rl}
0<f \leq 5 & W(f)=(500) / N F \\
5<f \leq 40 & W(f)=\left(12500 / f^{2}\right) / N F \\
40<f \leq 100 & W(f)=\left(3.2 \times 10^{10} / f^{6}\right) / N F
\end{array}
$$

Normalizing factor (NF) $=20,237$

ISO:

Vertical:

$$
\begin{array}{ll}
0<f \leq 1 & W(f)=(.1738) / N F \\
1<f \leq 4 & W(f)=(.1738 f) / N F \\
4<f \leq 8 & W(f)=(.6952) / N F \\
8<f \leq 100 & W(f)=\left(44.493 / f^{2}\right) / N F
\end{array}
$$

Normalizing factor (NF) $=9.386$

Transverse:

$$
\begin{array}{lr}
0<f \leq 2 & W(f)=(4.0) / N F \\
2<f \leq 100 & W(f)=\left(16.0 / f^{2}\right) / N F \\
\text { Normalizing factor (NF) }=16.12
\end{array}
$$


Table: 5.1. Frequency Weighting Functions (continued) (frequency in hertz)

UTACV:

Vertical:
$0<f \leq 1$
$W(f)=(1250) / N F$
$1<f \leq 4$
$W(f)=\left(1250 f^{.7702}\right) / N F$
$4<f \leq 6$
$W(f)=\left(f^{5.9139}\right) / N F$
$6<f \leq 25$
$W(f)=(40,000) / \mathrm{NF}$
$25<f \leq 50$
$W(f)=\left(2.5 \times 10^{7} / f^{2}\right) / N F$

Normalizing factor (NF) $=1.3013 \times 10^{6}$

Transverse:
$0<f \leq 1$
$W(f)=(1666) / N F$
$1<f \leq 4$
$W(f)=\left(1666 f^{.7925}\right) / N F$
$4<f \leq 6$
$W(f)=\left(.178 f^{7.388}\right) / N F$
$6<f \leq 25$
$W(f)=(100,000) / \mathrm{NF}$
$25<f \leq 50$
$W(f)=\left(6.25 \times 10^{7} / f^{2}\right) / \mathrm{iHF}$

Normalizing factor (NF) $=3.23116 \times 10^{6}$ 

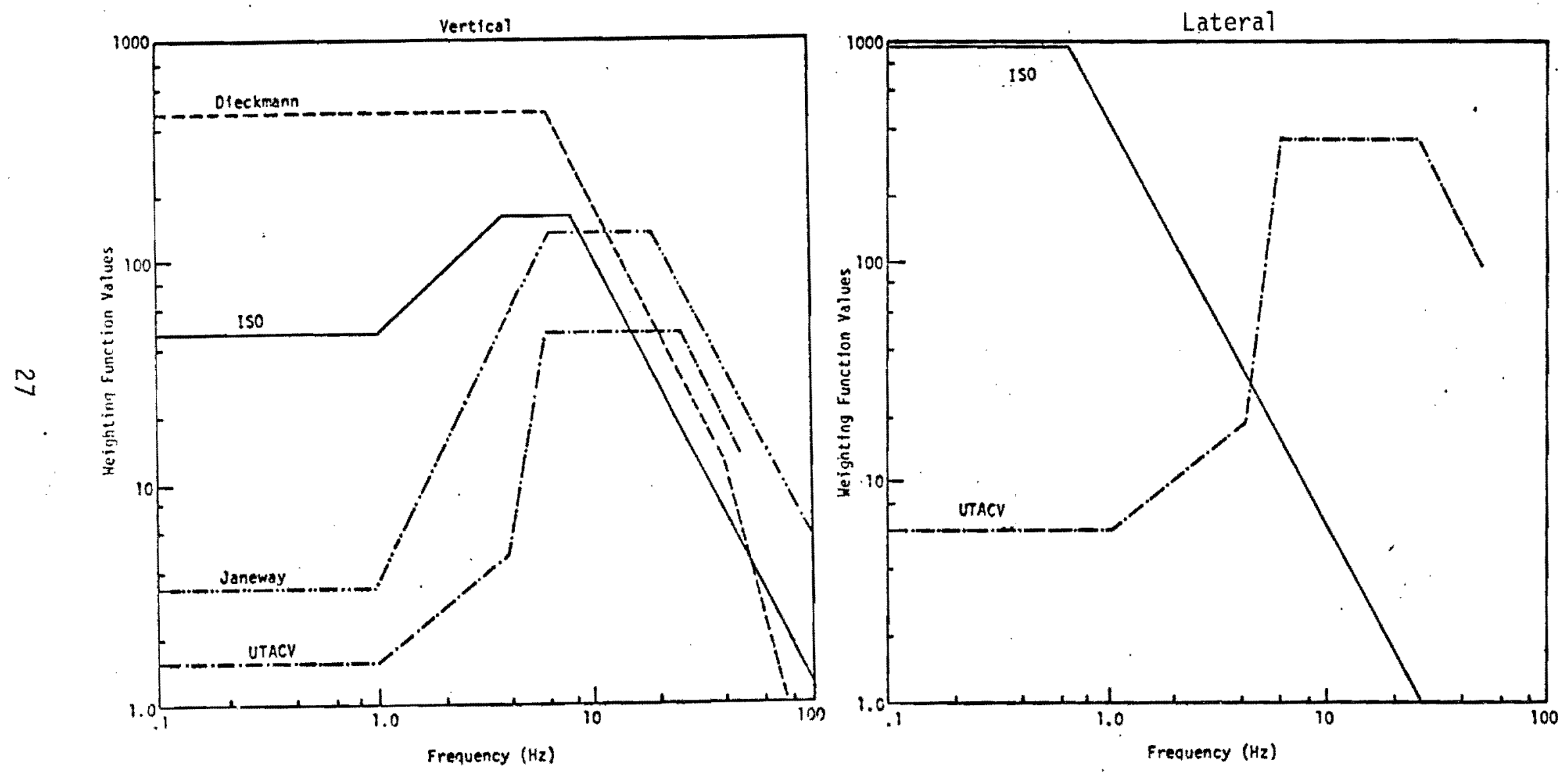

Figure 5.1. Frequency Weighting Functions 


\subsection{Absorbed Power}

The method relating to absorbed power proposed by Pradko and Lee is very simtlar to the frequency weighting scheme above. To consider the power absorbed by the passenger as an indicator of ride quality, the power absorbed at each point where the passenger contacts the vehicle can be calculated as

$$
A P_{i}=\int_{0}^{f_{i m}}\left|z_{i}(f)\right|^{2} P_{i}(f) d f
$$

where the subscript $i$ denotes the $i^{\text {th }}$ contact point, $z_{i}(f)$ is the impedance of the human body at the contact point, and $P_{j}(f)$ is the power spectral density of the vehicle vibration at the contact point. For practical reasons it is also assumed that all significant power is contained within the frequency band o to $f_{m}$ hertz. Comparing (5.3) with (5.1) it is noted that $\left|z_{j}(f)\right|^{2}$ can be considered as a weighting function similar to those previously discussed; however, the weighted index (in this case, the absorbed power) of the ride is the integral of the weighted spectrum rather than the square root of the integral. The absorbed power is therefore a weighted mean square measure rather than a weighted root mean square measure of the vibration. Weighting values for whole body and foot vibrations taken from [3] and used in this study are shown in Figure 5.2 and the equations are tabulated in Table 5.2.

To rigorously apply the absorbed power criterion, the vibration spectra and body impedance at each point of body contact with the vehicle would have to be known. Since only floorboard and seat spectra were measured in the case of the Maverick, the absorbed power criterion was applied in two ways. First, for both the Buick and the Maverick, the floorboard spectra were weighted using the sum of the whole-body and the foot weighting values for the vertical direction while the whole body weighting values were used for the lateral direction. The implicit assumption is that both the feet and the whole body are being vibrated with the floorboard. Both vertical and lateral absorbed power components were 

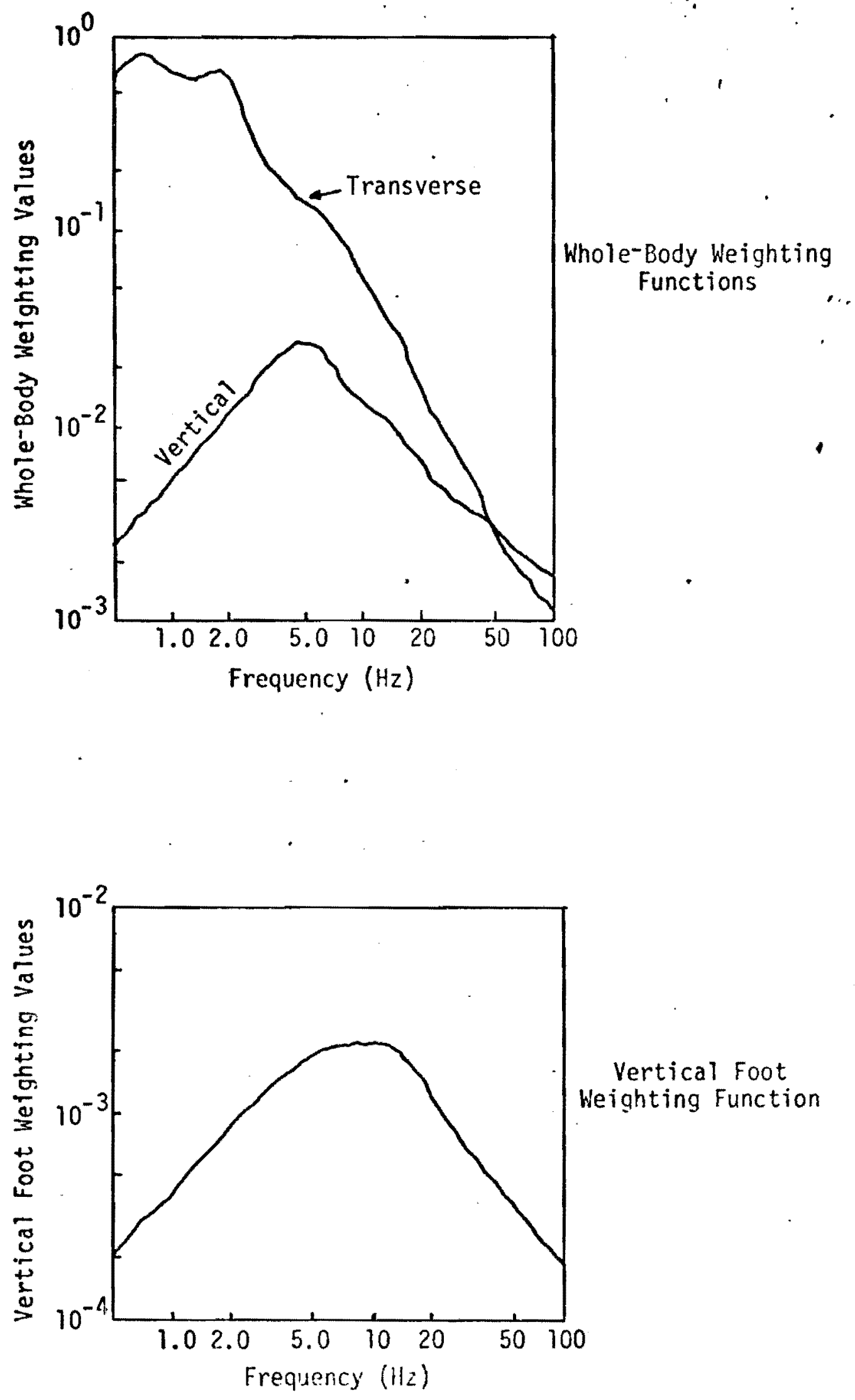

Figure 5.2. Absorbed Power Weighting Values 
Table 5.2. Absorbed Power Weighting Functions

Power $=\int_{0}^{100} R(f) P(f) d f$ (for given direction and location)

$R(f)=1.356 K_{d}\left[\frac{F l(f) F 4(f)-F 3(f) F 2(f)}{F 3(f)^{2}+f^{2} F 4^{2}}\right]$ watts $/ g^{2}$

Values for $k_{d}$ :

$$
\begin{aligned}
\text { Vertical whole body } K_{d} & =4.3537 \\
\text { Transverse whole body } K_{d} & =4.3530 \\
\text { Vertical foot } K_{d} & =1.182
\end{aligned}
$$

Values of $F 1, F 2, F 3, F 4$

functions of frequency $\mathrm{f}(\mathrm{Hz})$

Vertical Whole Body:

$F l=-.102453 \times 10^{-9} \times f^{6}+.175833 \times 10^{-5} \times f^{4}-.446007 \times 10^{-2} \times f^{2}+1.0$

$F 2=.128819 \times 10^{-7} \times f^{4}-.93394 \times 10^{-4} \times f^{2}+.10543$

$F 3=-.45416 \times 10^{-9} \times f^{6}+.37667 \times 10^{-5} \times f^{4}-.56104 \times 10^{-2} \times f^{2}+1.0$

$F 4=-.211792 \times 10^{-11} \times f^{6}+.5172811 \times 10^{-7} \times f^{4}-.17947 \times 10^{-3} \times f^{2}+.10543$

Transverse Whole Body:

$F 1=.24052 \times 10^{-3} \times f^{4}-.06697 \times f^{2}+1.0$

$F 2=.5738 \times 10^{-5} \times f^{4}-.5017 \times 10^{-2} \times f^{2}+.330926$

$F 3=-.1498 \times 10^{-5} \times f^{6}+.0010089 \times f^{4}-.101087 \times f^{2}+1.0$

$F_{4}=-.171375 \times 10^{-7} \times f^{6}+.53137 \times 10^{-4} \times f^{4}-.0110965 \times f^{2}+.330926$

Vertical Foot:

$F 1=.58657 \times 10^{-7} \times f^{4}-.188245 \times 10^{-2} \times f^{2}+1.0$

$F 2=-.1870696 \times 10^{-4} \times f^{2}+.074037$

$F 3=.339132 \times 10^{-6} \times f^{4}-.236976 \times 10^{-2} \times f^{2}+1.0$

$F 4=.170135 \times 10^{-8} \times f^{4}-.3944 \times 10^{-4} \times f^{2}+.074037$ 
tabulated as well as the sum of the two. The second way the criterion was applied used the Maverick data only and weighted the seat vibrations via the whole body weighting values while the vertical floorboard spectra were weighted via the foot vibrations. Again, vertical (foot and whole body) and lateral (whole body only) components were tabulated along with their sum. The resulting absorbed power "indices" are tabulated with the other weighted ride indices in Appendix D.

\subsection{Unweighted RMS Acceleration Ride Indices}

In addition to the frequency weighted ride indices described above, another plausible ride index is simply the "unweighted" rms value of the ride acceleration as defined by equation 2.3. This is obviously also equivalent to using a weighting function $W(f)=1.0$ which gives all frequencies equal weight. Two ride indices of this type were also considered in this study, one which considered all frequency components up to $f_{m}=100$ hertzand one which considered components only up to $f_{m}=40$ hertz. By comparison of these two ride indices, indication of the relative importance of components between 40 and 100 hertz and those below 40 hertz is inferred. These ride indices are also tabulated in Aopendix E.

\subsection{Correlation Study}

Using each of the weighting schemes described above, weighted indices were calculated for vertical and lateral vibrations for each of the rides corresponding to the rating sessions described in Chapter 3 . Indices were calculated using the measured floorboard vibrations for both the Buick and the Maverick and the measured seat vibrations for the Maverick. Additionally, where both vertical and lateral weighting functions exist, a "magnitude" index, defined as the square root of the sum of the squares of the vertical and lateral ride indices was calculated. As indicated previously in section 5.1 , no attempt was made to obtain an optimum relative weighting between vertical and lateral effects in calculating this magnitude index. 
After calculation of the above described indices, a correlation study was performed to determine the correlation of the indices using each different weighting scheme with the mean personal ratings of the rides. The higher the degree of correlation between the weighted indices and the mean personal ratings, the better the weighted index is as a predictor of mean personal rating and hence the quality of the ride. Pearson product-moment correlation coefficients for correlation of each of the ride indices with corresponding mean personal ratings are tabulated in Table 5.3. Additionally, indices for each weighting scheme are plotted as a function of corresponding mean personal rating in Appendix $E$, allowing graphical examination of which weighting method best collapses the data.

\subsection{Discussion of Statistical Results}

In presenting the results of the study many different aspects of the data should be considered. The main points to be made are that frequency weighting techniques showed little if any improvement over the unweighted RMS values and that the position from which the measurements were taken (seat versus floor) revealed that vertical vibration was the predominant indicator for the floor vibration and lateral vibration was the dominant indicator of ride quality for the seat vibration. In the following discussion, it will be considered that any correlation coefficient of .90 or greater indicates good predictability.

5.5.1 Frequency Weighting Versus Unweighted Values The main result, which is contrary to the expected, is that in general the frequency weighted RMS values did not predict ride quality better than unweighted RMS values. This is easily seen in Figures 5.3, 5.4, 5.5 and 5.6. The raw acceleration values of vertical, lateral, and magnitude (the square root of the sum of the squares of vertical and lateral) were generally as good as the results using the weighting functions. The good predictability group for vertical floor vibrations for both Buick and Maverick includes Absorbed Power (.95), Janeway (.93), UTACV (.90), Dieckmann (.91), ISO (.92), RMS 40 (.92) and RMS 100 (.92), and for vertical seat 
Table 5.3. Correlation Coefficients

\begin{tabular}{|c|c|c|c|c|}
\hline $\begin{array}{c}\text { Maverick } \\
\text { Seat }\end{array}$ & $\begin{array}{l}\text { Combined } \\
\text { Floors }\end{array}$ & $\begin{array}{c}\text { Maverick } \\
\text { Floor }\end{array}$ & $\begin{array}{l}\text { Buick } \\
\text { Floor }\end{array}$ & \\
\hline .92 & .93 & .94 & .93 & Janeway--Vertical \\
\hline .88 & .91 & .99 & .87 & Dieckmann--Vertical \\
\hline .91 & .90 & .90 & .92 & UTACV--Vertical \\
\hline .95 & .62 & .84 & .90 & UTACV--Lateral \\
\hline .97 & .85 & .89 & .94 & UTACV--Magnitude \\
\hline .86 & .92 & .97 & .91 & ISO--Vertical \\
\hline .92 & .56 & .60 & .67 & ISO--Lateral \\
\hline .92 & .83 & .82 & .91 & ISO--Magnitude \\
\hline .89 & .92 & .99 & .90 & RMS 0 to $100--V$ ertical \\
\hline .98 & .62 & .79 & .85 & RMS 0 to 100 --Lateral \\
\hline .99 & .92 & .95 & .92 & RMS 0 to 100 --Magnitude \\
\hline .87 & .92 & .99 & .89 & RMS 0 to $40--$ Vertical \\
\hline .98 & .66 & .81 & .89 & RMS 0 to 40 -Latera 1 \\
\hline .98 & .92 & .95 & .91 & RMS 0 to $40--$ Magnitude \\
\hline $.90^{*}$ & .95 & .98 & .95 & Absorbed Power*--Vertical \\
\hline $.87^{\star}$ & .51 & .68 & .62 & Absorbed Power*--Lateral \\
\hline $.89^{*}$ & .73 & .79 & .94 & Absorbed Power*--Total \\
\hline
\end{tabular}

*These correlations refer to absorbed power using seat vibrations as whole body input and floorboard vibrations as foot input. 


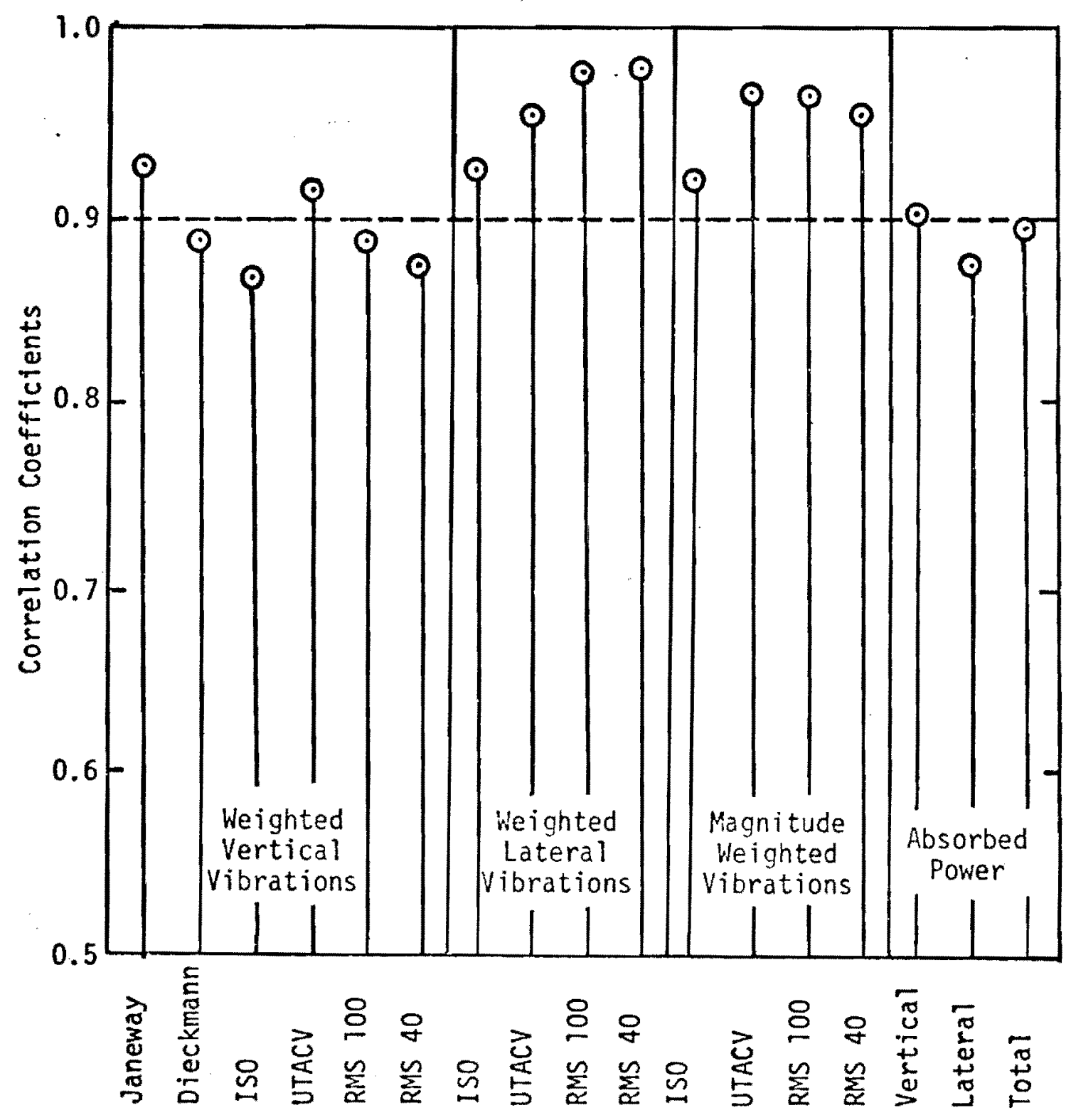

Figure 5.3. Correlation of Weighted Indices for Maverick Seat Vibrations with Mean Personal Ratings. 


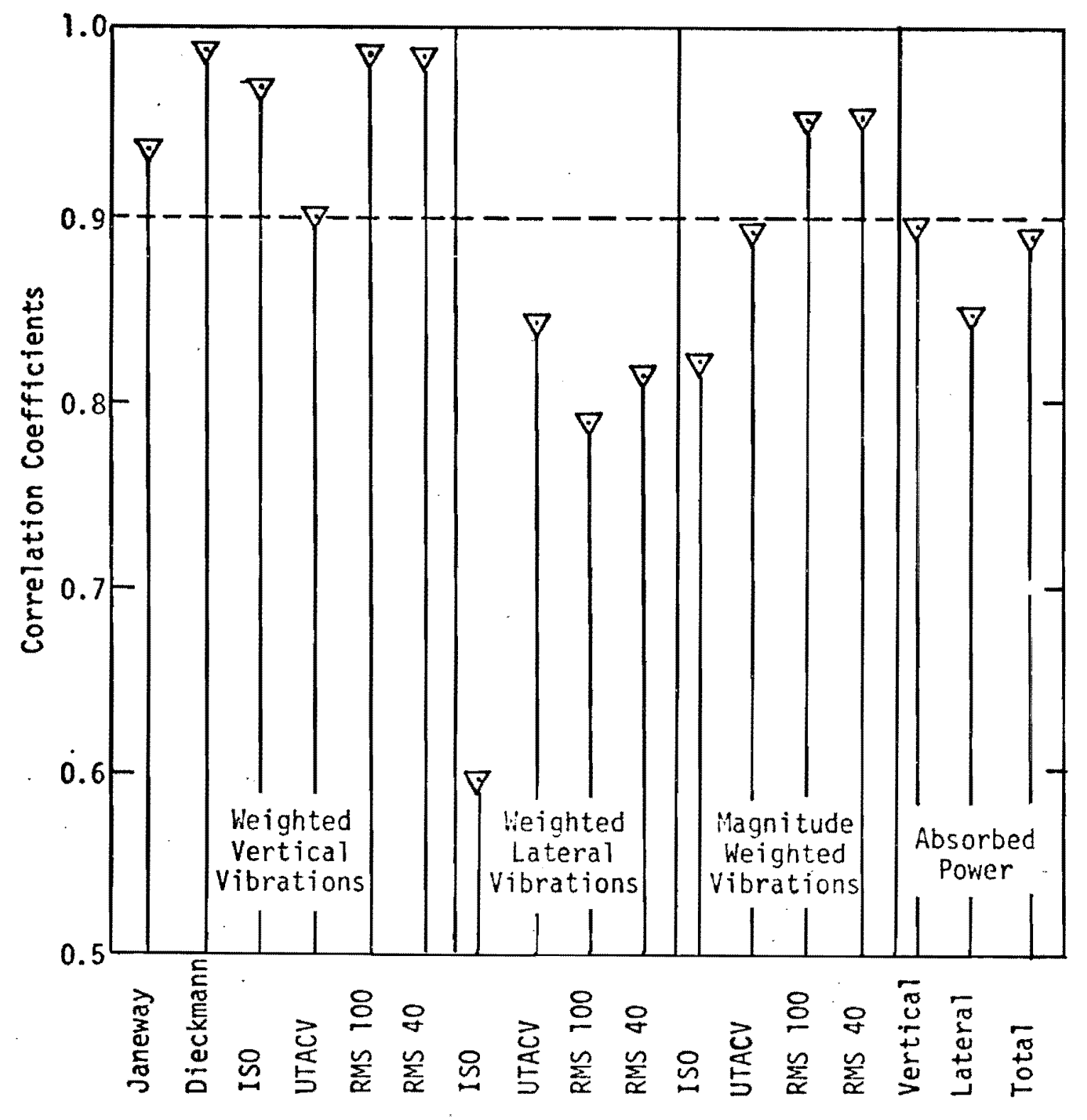

Figure 5.4. Correlation of Weighted Indices for Maverick Floor Vibrations with Mean Personal Ratings. 


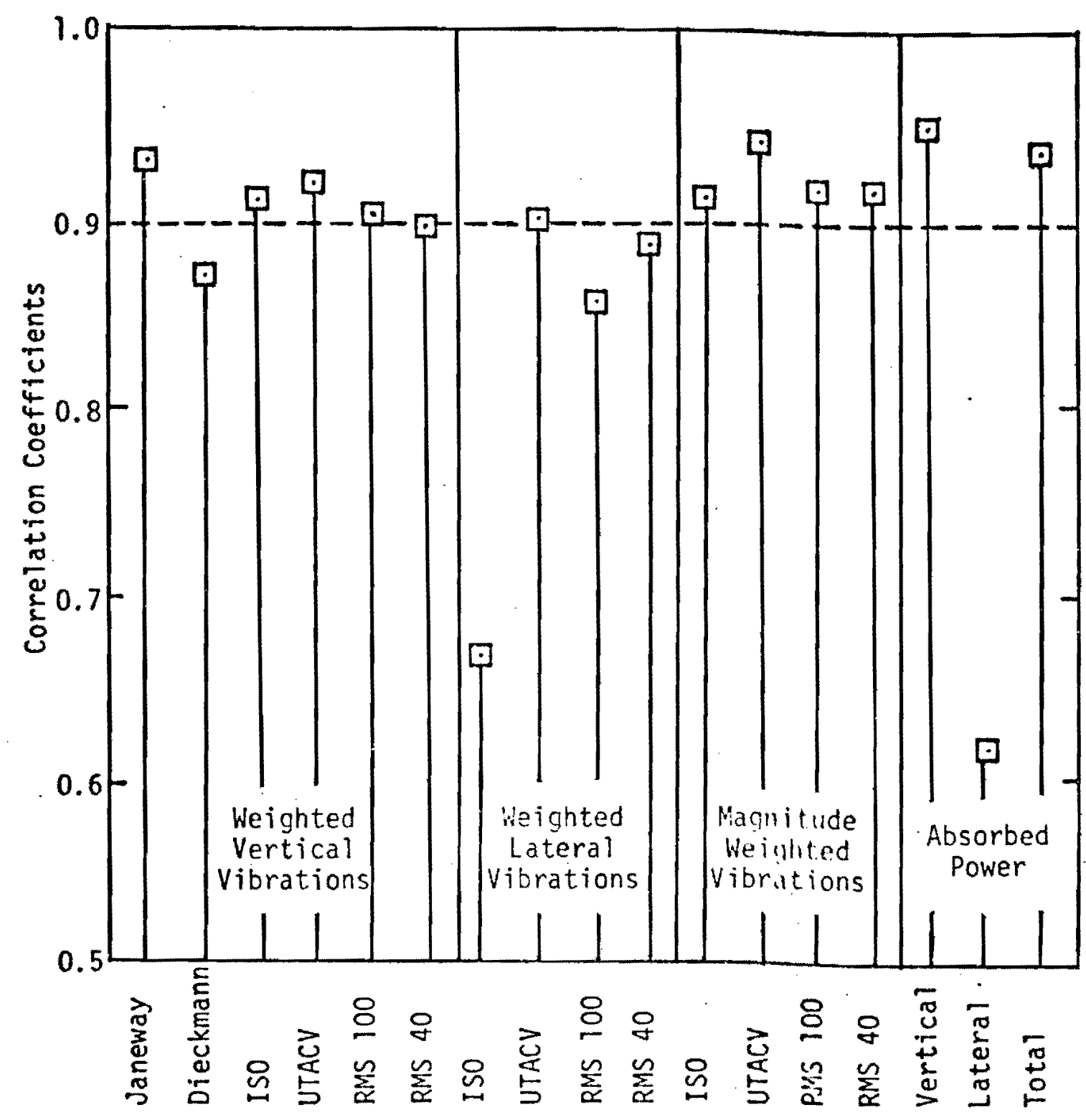

Figure 5.5. Correlation of Weighted Indices for Buick Floor Vibrations with Mean Personal Ratings. 


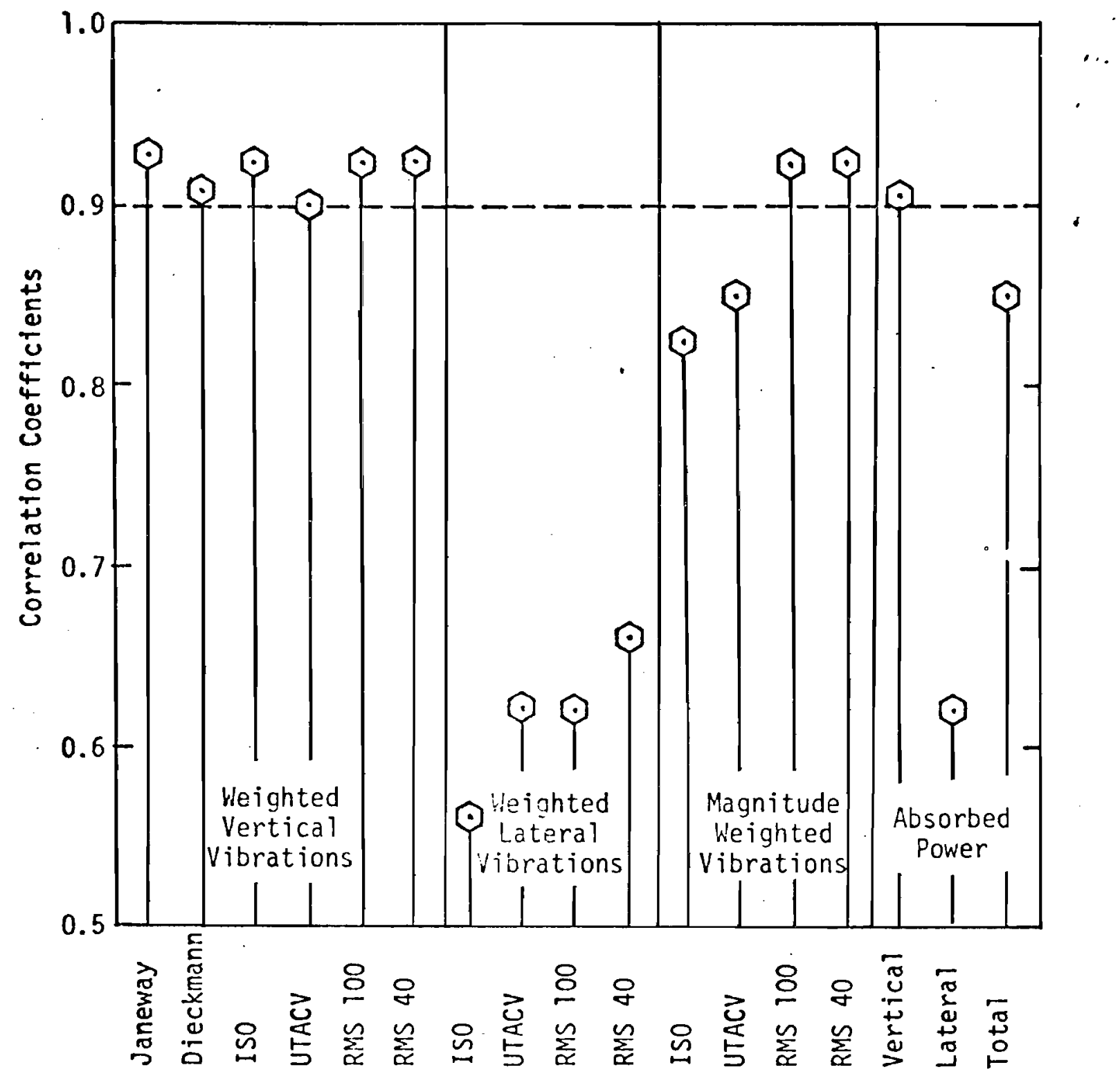

Figure 5.6. Correlation of Weighted Indices for Combined Buick and Maverick Floorboard Vibrations with Mean Personal Ratings. 
vibrations Janeway (.92) and Absorbed Power (.90) were the good predictors. In the lateral direction, there was no good predictor using floor vibrations; however, for the seat, ISO (.92), RMS $100(.98)$ and RMS $40(.98)$ were considered good. In considering the magnitude (the square root of the sum of the squares) of the vertical and transverse directions for floorboard calculations, only (unweighted) RMS $40(.92)$ and RMS $100(.92)$ ranked as good predictors. Combining the vertical and transverse results of the Maverick seat into a magnitude yielded good predictability from ISO (.92), UTACV (.97), RMS 100 (.99), and RMS $40(.98)$.

From the above results, unweighted RMS values appear to be the most logical choice for ride predictors. This is apparent since the weighting functions provided little if any improvement for any of the situations considered. An explanation of this unexpected result might be that since the spectra for all rides contained about the same relative frequency composition, the weighting schemes were not effective. Indeed the raw correlations are good enough that one would not expect significant improvement from any weighting scheme. It should be emphasized that tests were run in two different size automobiles and that the combined data of the two cars yielded results very close to the individual results. This would lead one to conclude that the results would apply similarly to automobiles in general.

\subsubsection{Significance of Location of Measurement and Direction of Vibration}

Also to be considered is the location and direction of the inputs. Locations used were floorboard and seat while directions used were vertical and lateral. Although the intuitive tendency would be to discuss these separately, the results of location and direction of the measurements are so closely related that separate discussion would be either confusing or redundant. For consistency, this discussion will be concerned only with the floor and seat vibrations of the Maverick since seat measurements were not taken in the Buick. It is suggested that in order to best realize the results being discussed, the reader refer to Figures 5.3 and 5.4 . 
The most surprising result is the flip-flop of predictability from floor to seat vibrations and how this relates to the vibration direction. It can be seen that for the floor vibrations, the vertical vibrations are usually a good predictor while for the seat vibrations the lateral direction is the best predictor. From a review of the unweighted rms values in Appendix $D$, it is seen that the vertical vibrations were of greater magnitude relative to the lateral vibrations on the floorboard, while the lateral vibrations were dominant at the seat. For both seat and floor the magnitude unweighted RMS values are consistently good predictors, and the values at the seat and floorboards are approximately equal. Therefore, the vector sum (the square root of the sum of the squares) of the vertical and lateral values would be the best solution to using a uniform ride evaluation system.

5.5.3 Seat Versus Floorboard Measurements Another point of controversy in the past has been whether seat or floor measurements should be used to evaluate ride quality. In this study it is evident that either reveais a good estimate of how riders will rate the ride. However, for a more thorough understnading each component should be considered individually, then combined and evaluated as a system.

\subsubsection{Significance of Frequency Range Considered At this point the dis-} tinction between the frequency ranges of 0 to $40 \mathrm{~Hz}$ and 0 to $100 \mathrm{~Hz}$ should be mentioned. Overa11, there was little difference, leading one to believe that the frequencies beyond 40 hertz contain little information about the ride which is not contained in the components below 40 hertz. Examination of the data, however, indicated that for the rides considered there is a relatively small amount of spectral content beyond 40 hertz so part of the reason for its lack of importance may be due to the fact that it just isn't there. It is concluded 
that generally it would be satisfactory to evaluate ride using frequencies up to 40 hertz, although the added information of 0 to $100 \mathrm{~Hz}$ would be recommended if detailed analysis of the vehicle is desired.

\subsection{Summary}

In summary, the correlation study made the following conclusions apparent. With regard to floor vibrations, vertical vibration was dominant with various weighting functions acquiring good predictability along with unweighted RMS values. Likewise, seat data showed the unweighted RMS values for lateral motion to be good ride predictors. Overal1, however, the unweighted RMS accelerations within the 0-40 hertz and 0-100 hertz bands were consistently good predictors using both floor and seat vibrations. 


\section{RIDE EVALUATION EQUATIONS}

Since the results presented in the previous chapter indicate that there is a high degree of (linear) correlation between the weighted indices and the mean personal ratings, it is evident that the weighted indices can be used as predictors of the mean personal ratings. If $R$ is defined as the predicted MPR for a given index $\alpha$, then the linear relationship

$$
R=a+b a
$$

where $\mathrm{a}$ and $\mathrm{b}$ are constants, can be used to calculate $\mathrm{R}$ for any measured $\alpha$. From the data measured in this study, best fit values for the constants a (the intercept of equation 6.1) and $b$ (the gradient) for each of the weighting schemes are tabulated in Appendix E.2. "Best fit" values for a and b are defined here in a least squares sense as the values which minimize the residual variance

$$
\begin{gathered}
\sigma^{2}=\frac{1}{N} \stackrel{N}{\xi}\left(R\left(\alpha_{i}\right)-M P R_{i}\right)^{2} \\
i=1
\end{gathered}
$$

where $N$ is the number of data points and $M P R_{j}$ is the mean personal rating of a ride with index $\alpha_{i}$. Corresponding values of residual variances and standard deviations (defined as the square root of the variance) for each weighting scheme are also tabulated in Appendix E.2.

\subsection{Proposed Comfort Equation}

Because of their high correlation with MPR and their relative ease of application, the "unweighted" RMS ride indices were proposed in Chapter 5 for general use. It was also noted in Chapter 5 that the "magnitude" seat and floorboard vibrations are of about the same magnitude. As a result, if all the magnitude unweighted RMS from 0 to 40 hertz ride indices for seat and floorboard vibration are correlated with their corresponding mean personal ratings, 
the resulting correlation coefficient is 0.93 . This indicates that if the magnitude RMS index is used, the same linear equation (gradient and intercept) can be used for both floorboard and seat vibrations. For this case, the resulting least squares fit equation is

$$
R=5.43-40.0 \alpha
$$

where $\alpha$ is the magnitude RMS (the square root of the sum of the squares of the vertical and lateral RMS accelerations) acceleration at either the floorboard or the seat, and $R$ is the ride rating. The residual variance for this case is $\sigma^{2}=0.0667$ and the standard deviation $\sigma=0.26$. In Figure 6.1 the data points and corresponding line are plotted to illustrate the resulting curve fit and corresponding spread of the data.

If the RMS accelerations are calculated using spectra to 100 hertz rather than 40 hertz, the correlation and fit equation changed only slightly, the improvement being insignificant. Therefore equation (6.3) is recommended as the best overall measure of ride rating as a function of the riding vibrations.

\subsection{Discomfort Equation}

Since equation (6.3) results in a measure of the ride which decreases as a function of the level of vibrations, it makes some intuitive sense to convert (6.3) to the form of a discomfort equation such that

$$
D=5.0-R
$$

where $D$ is defined as the discomfort index of the ride. Substituting (6.3) into $(6.4)$

$$
D=-0.43+40.0 \alpha
$$

where now D will vary roughly from about 0 ("the best ride you can think of") to 5 ("the worst ride you can think of"). Comparing the values of D with the road sections rated in this study we find that: $D<1.0$ corresponds roughly to 


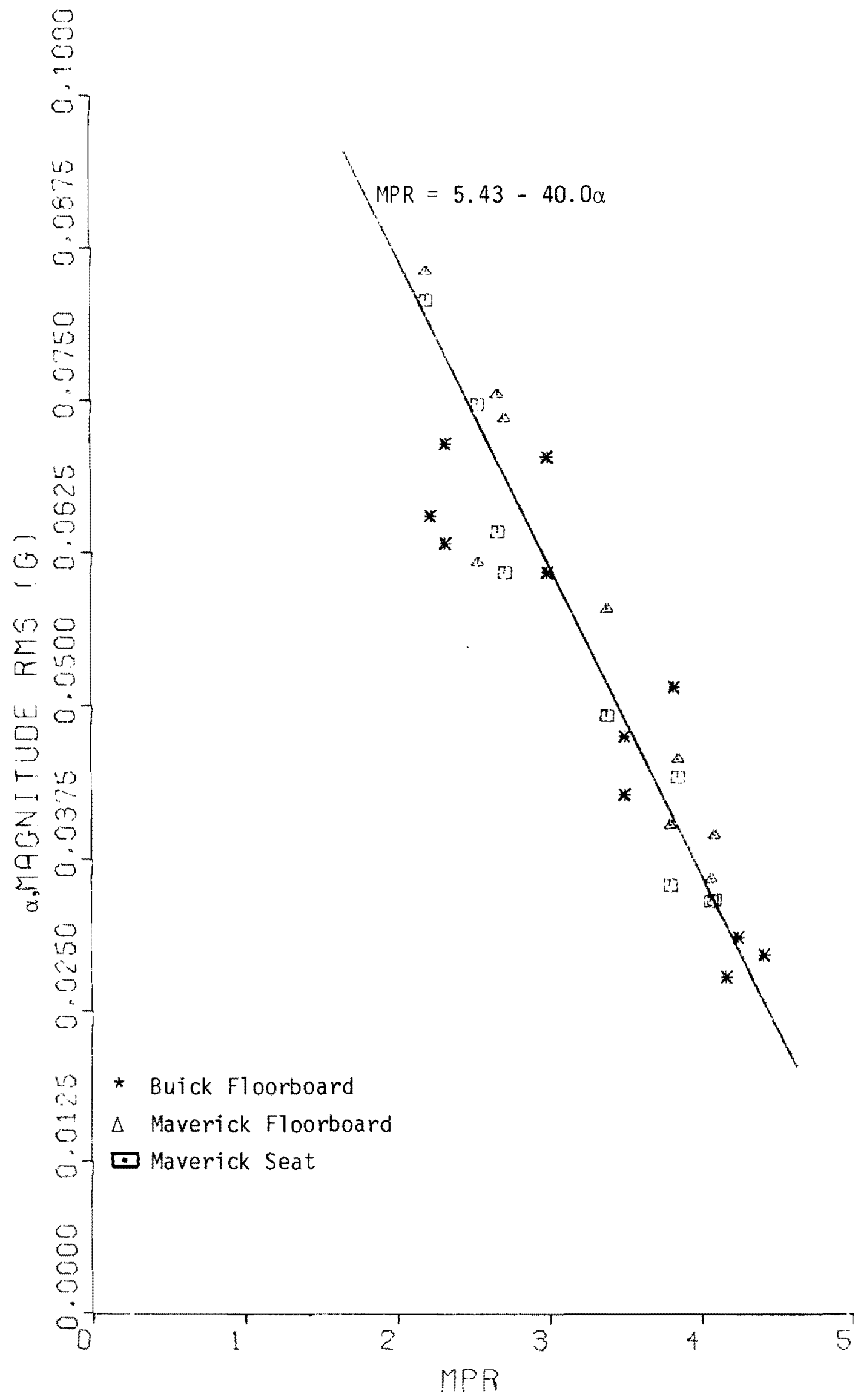

Figure 6.1 Least Squares Curve Fit to Magnitude RMS Acclerations Within the 0 to 40 Hertz Band vs. Mean Personal Ratings. 
an automobile ride on a very smooth interstate highway, $1<0<2$ to an automobile ride on a typical state highway, and $2<D<3$ to an automobile ride on a rough secondary road. The residual variance and standard deviation of the discomfort index $D$ are the same values as for the ride rating $R$. 


\section{CONCLUSIONS AND RECOMMENDATIONS}

This study has approached the problem of evaluating the ride quality in automobiles using spectral analysis of the actual vehicle vibrations, with the main emphasis being the comparison of different frequency weighting techniques. Seventy-eight subjects were driven over eighteen different road sections in two different automobiles in order to obtain reliable subjective responses to ride quality in automobiles. The resulting ratings and the vehicle vibration spectra were compared graphically to the ISO reduced comfort boundary and the UTACV specification boundary. Various methods of frequency weighting techniques were applied to the vehicle vibration spectra and indices obtained using each weighting technique. The various weighting techniques were then compared by studying the resulting correlation of the indices using a particular weighting technique and the mean personal (subjective) rating of the passengers. Unweighted RMS values were consistently as good of predictors of ride quality for both seat and floorboard vibration as the best weighted RMS values, and are simpler and easier to use.

As a result of this study, it seems proper to present the following conclusions and recommendations. First, in evaluating the ride of automobiles, spectral analysis of the actual vehicular vibration is a very useful tool. The evaluation of the vibrations could be done using unweighted acceleration spectra for floor or seat data in the vertical and transverse directions. A magnitude of the RMS values defined as the square root of the sum of the square of the vertical and lateral RMS acceleration is recommended for either of the locations. The values of these magnitude weighted rms values will range roughily from 0 to $0.04 \mathrm{~g}$ for smooth (interstate highway) rides, 0.04 to $0.06 \mathrm{~g}$ for medium rides, and above 0.06 for rough rides which could be used to predict statistically general passenger rating of the ride as presented in Chapter 6. 
Since this study involved the use of different automobiles and a variety of spectra, these results are recommended for use with automobiles in general. With regard to vehicles with vibration spectra significantly different from those of automobiles, caution is advised in directly applying the results of this study. However, a similar approach might be used to obtain criteria for other types of vehicles before any attempt is made to produce a universal ride comfort criterion. 


\section{REFERENCES}

1. Roberts, F. L., and Hudson, W. R., "Pavement Serviceability Equations Using the Surface Dynamics Profilometer, Research Report 73-3, Center for Highway Research, The University of Texas at Austin, April 1970.

2. Anonymous, "A Guide to the Evaluation of Human Exposure to Whole Body Vibration", ISO/DIS 2631, International Organization for Standardization, New York, 1972 .

3. Lee, R. A., and Pradko, F., "Analytical Analys is of Human Vibration", S.A.E. Transactions 680091, 1968, pp. 346-370.

4. Anonymous, "Design Specifications for Urban Tracked Air Cushion Vehicles", U. S. Department of Transportation, Washington, D. C., 1972.

5. Janeway, R. N., "Vehicle Vibration Limits to Fit he Passenger", S.A.E. J., Volume 56, August, 1948, pp. 48-49.

6. Dieckmann, D., "Einfluss Vertikaler Mechanischer Schwingungen auf den Menschen", Inernat. Z. Angew-Physiol. 16, 1957, pp. 519-564.

7. Healey, A. J., "Passenger Response to Random Vibration in Transportation Vehicles - A Literature Review", Research Report RR-30, Council for Advanced Transportation Studies, University of Texas, June 1975.

8. Butkunas, A. A., "Power Spectral Density and Ride Evaluation", S.A.E. Transactions No. 660138,1966, p. 681-687.

9. Spangler, E. B. and Kelly, W. J., "GMR Road Profilometer - A Method for Measuring Road Profiles", Research Report GMR-452, General Motors, December, 1964.

10. Smith, C. C. "On Using the ISO Standard to Evaluate the Ride Quality of BroadBand Vibration Spectra in Transportation Vehicles", ASME Transactions, Journal of Dynamic Systems, Measurement and Control, Vo1. 98, Series G, No. 4, December, 1976. 


\section{COMPUTATION OF POWER SPECTRAL DENSITY}

\section{A.1. Introduction}

The signals processed in this work were measured acceleration time series, with sampled values at discrete intervals. A measure of the frequency content of the random signals is provided by estimation of their power spectral density (PSD). The PSD is an averaged measure of the square of the signal amplitude contained in a narrow frequency band divided by the bandwidth. For each acceleration trace, a total of 4096 data points were taken at a sampling rate of 434 hertz, corresponding to about 9.44 seconds of data per trace.

\section{A. 2 Detrending}

Since the profiles of the roadway test sections used contain no significant grades, the linear trend of the acceleration traces is small. Care was taken, however, to insure that the acceleration traces had zero mean. This was accomplished by the operation

$$
x_{k}=\left(x_{k}\right)_{01 d}-\bar{X}
$$

where the mean $\bar{X}=\frac{1}{N} \sum_{K=0}^{N-1}\left(X_{k}\right)$ old 
The resulting sequence

$$
\left(x_{0} \cdot \cdot x_{k} \cdot \cdot x_{N-1}\right)
$$

is analyzed for its power spectral composition. The auto correlation defined as

$$
C(r)=\frac{1}{N} \sum_{k=0}^{N-1} x_{k} \cdot x_{k+r}
$$

\section{A.3 Power Spectrum Calculations}

The two sided power spectral density is then given by the discrete Fourier transform of $C(r)$.

$$
G(k)=\frac{1}{N} \sum_{r=0}^{N-1} C(r) e^{-j 2 \pi r k / N}
$$

If the original sequence $x_{k}$ is real, $P(k)$ will be complex with a real and imaginary part being symmetric and anti-symmetric respectively, about the $N / 2$ point.

Taking advantage of the Fast Fourier Transform Algorithm ${ }^{14}$, it is better to compute the FFT of $x_{k}$, using the property that the transform of a convoluted sequence is the product of the individual transforms with its conjugate so that if

$$
x_{(k)}=\frac{1}{N} \sum_{r=0}^{N-1} x_{r} e^{-j j^{2 \pi r k / N}}
$$

the power density is

$$
G_{(k)}=\left(X_{k} X_{k}^{*}\right) T_{r}
$$

where $T_{r}=$ the total time of the trace included to reconstitute dimensional units in the power function. The one sided power spectral density is then 
defined as

$$
P(k)=2 G(k) \quad k=0,1, \ldots, \frac{N}{2}
$$

\section{A.4 Data Averaging}

The sampling frequency for the data was 434 hertz and the incremental discretion frequency was about 0.106 hertz. Averaging over $d$ incremental bands yielding $d$ degrees of freedom for each averaged power computation, the power spectral sequence

$$
p_{0}--p_{k}--p_{n}
$$

converts to the data smoothed sequence

$$
\hat{p}_{d}-\hat{p}_{k}--\hat{p}_{n-1-d} \text {. }
$$

According to

$$
\hat{p}_{k}=\frac{1}{(2 d+1)_{k-d}^{k+d}} p_{k} \quad k=d \text { to } N-1-d
$$

The frequency associated with $\hat{p}_{k}$ still remains at $k / L$ cycles/ft.

While equation (9) smoothes the data, total power is not conserved in the smoothing process. The errors are introducted by the failure to include points in the smoothed array for $k<d$ and $k>(N-1-d)$. With typical spectra this error is sma 11. Total power error is given by

$$
\text { Total Power Error }=\frac{1}{(2 d+1)} \sum_{\dot{\lambda}=0}^{\lambda=2 d}(2 d-\lambda)\left(p_{\lambda}+p_{N-1-\lambda}\right)
$$


APPENDIX B

RAT ING FORM

1. How would you rate the car ride you have just taken?

$\begin{array}{lllllll}\text { worst ride I } & 1 & 2 & 3 & 4 & 5 & \text { best ride I }\end{array}$

2. How would you rate your mood right now?

$\begin{array}{lllllll}\text { worst mood I } & 1 & 2 & 3 & 4 & 5 & \begin{array}{l}\text { best mood I } \\ \text { can think of }\end{array}\end{array}$

3. How would you rate the weather right now?

$\begin{array}{lllllll}\text { worst weather } & 1 & 2 & 3 & 4 & 5 & \text { best weather }\end{array}$

I can think of I can think of

Your name

date

section number 
APPENDIX C

SPECTRAL DENSITY PLOTS 

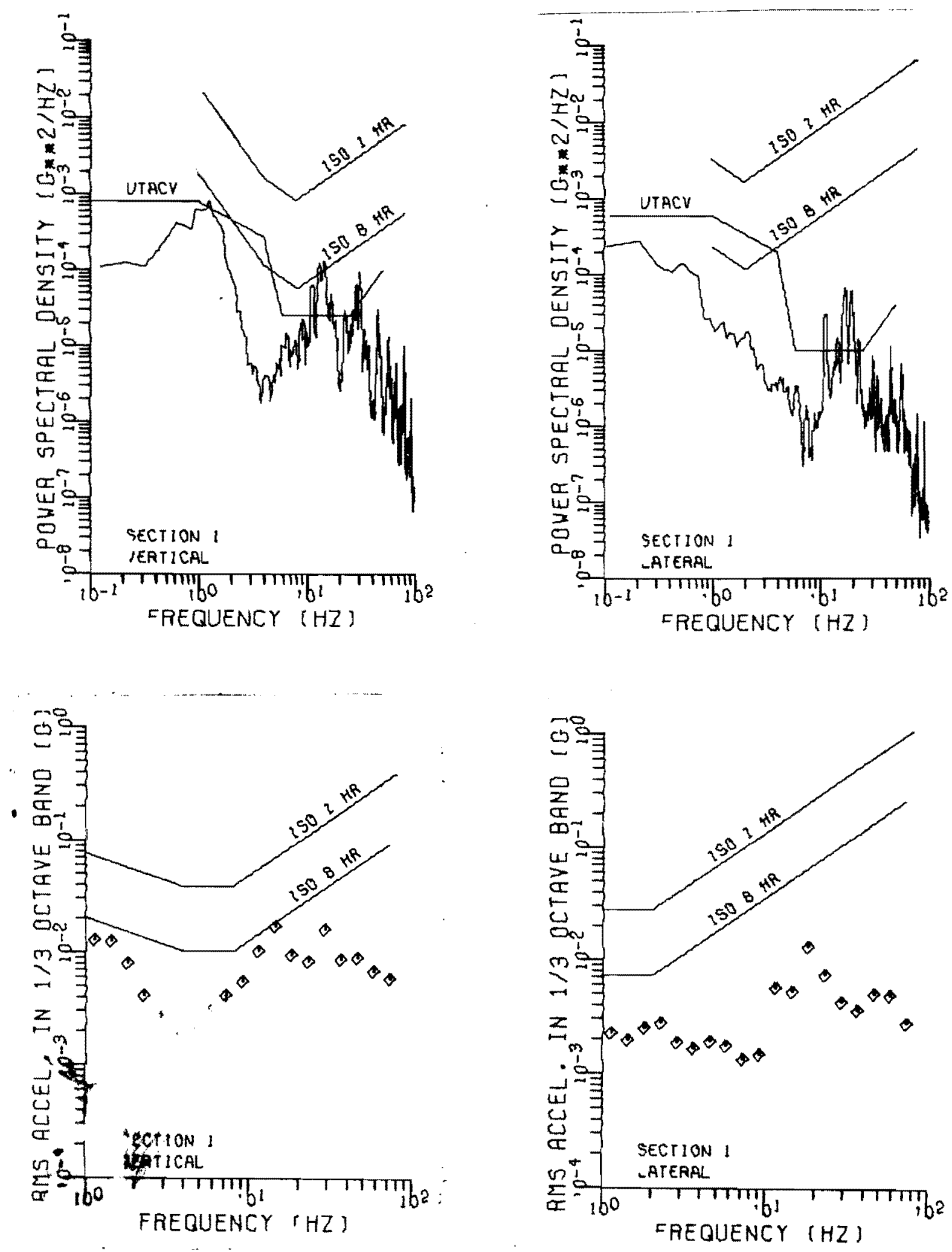

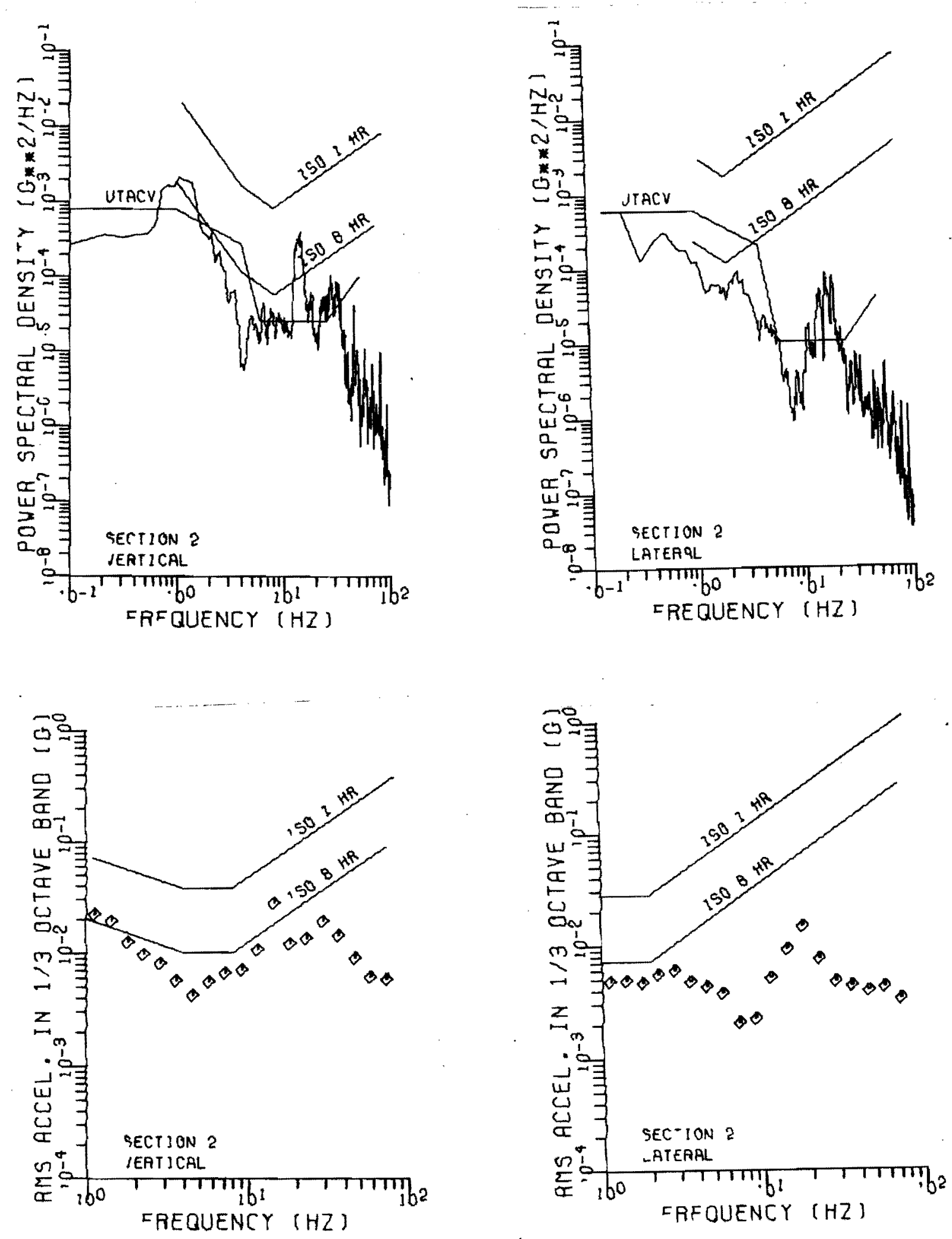


\section{BUICK FLOOR VIBRATIONS}
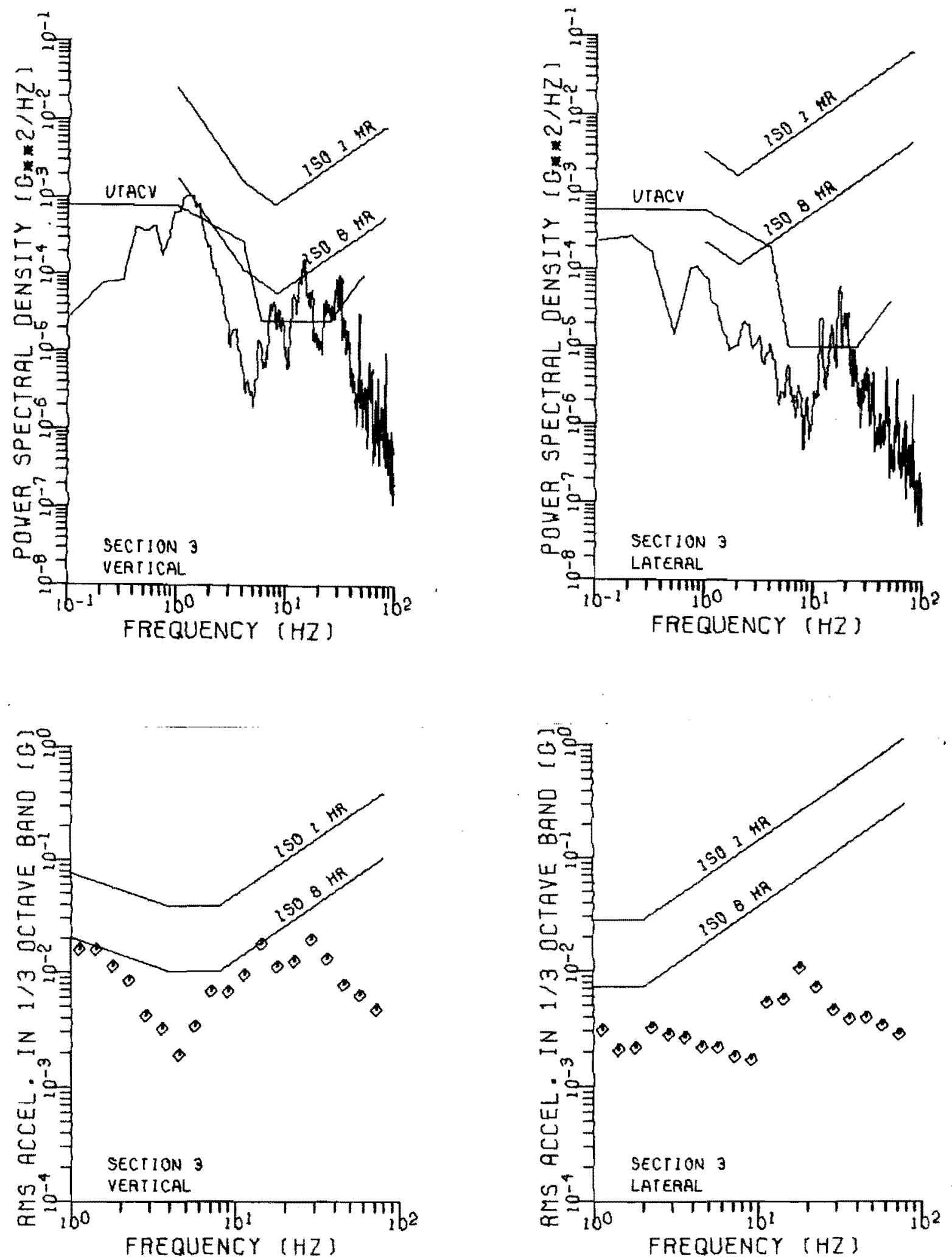

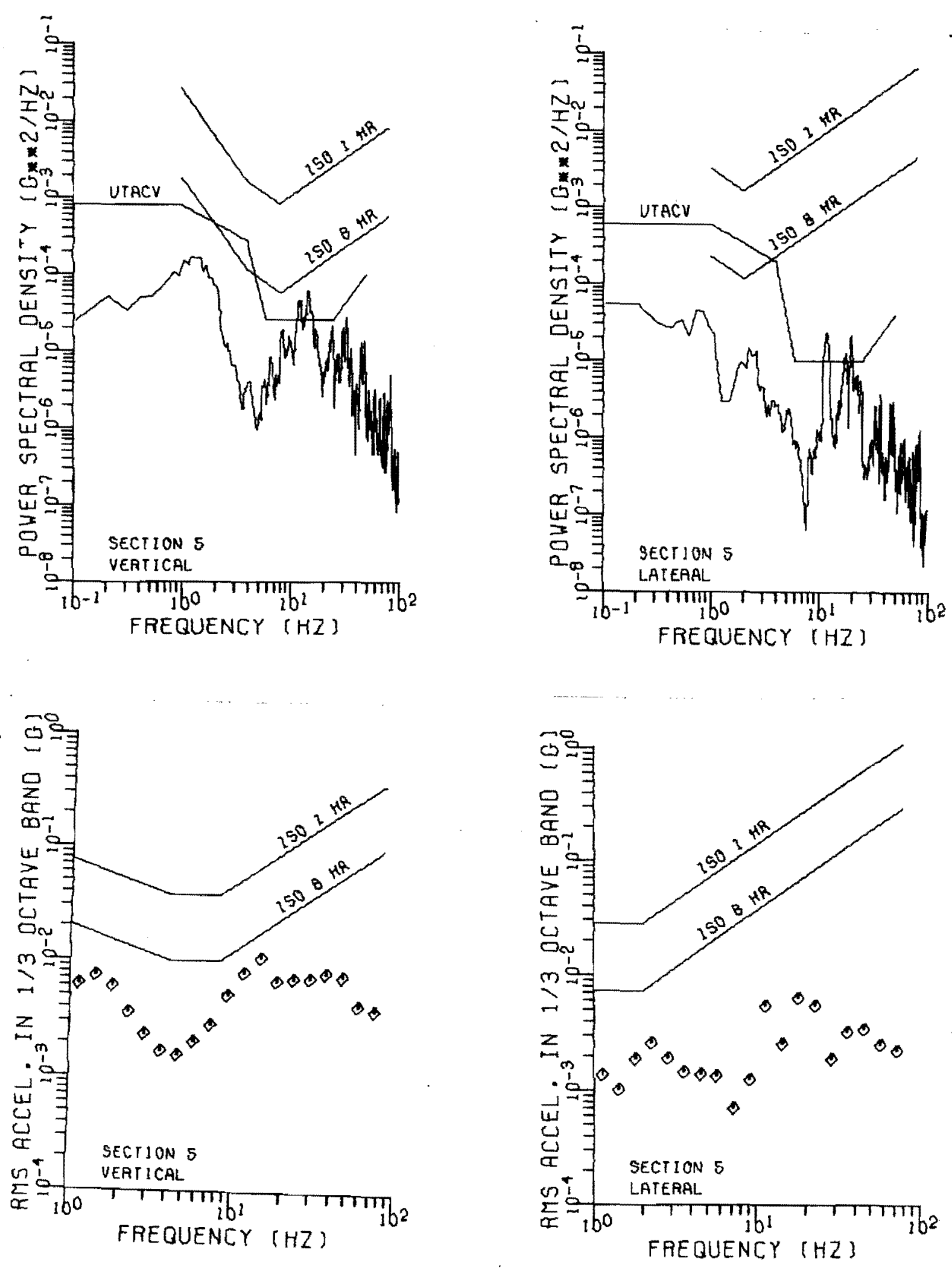

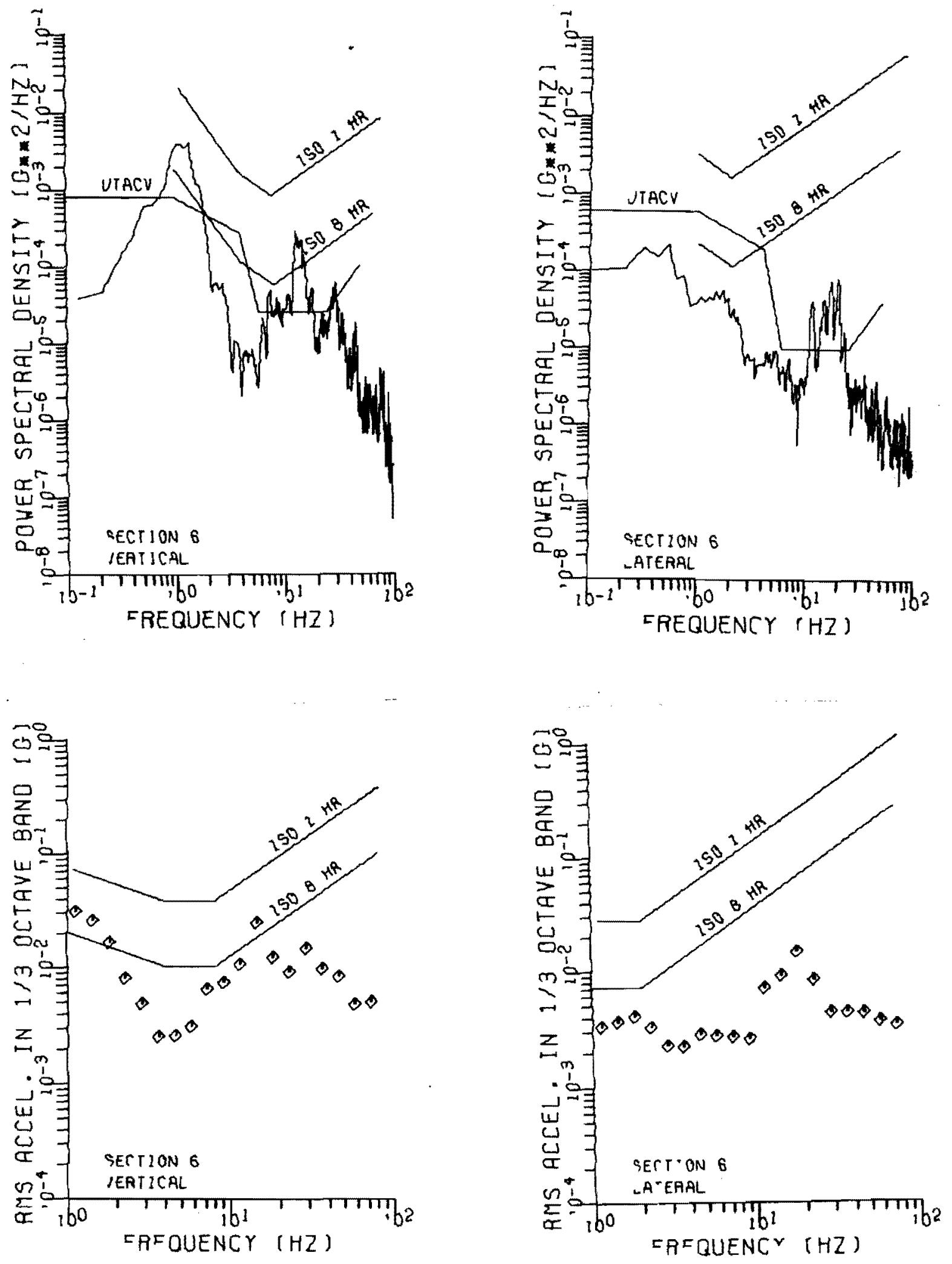

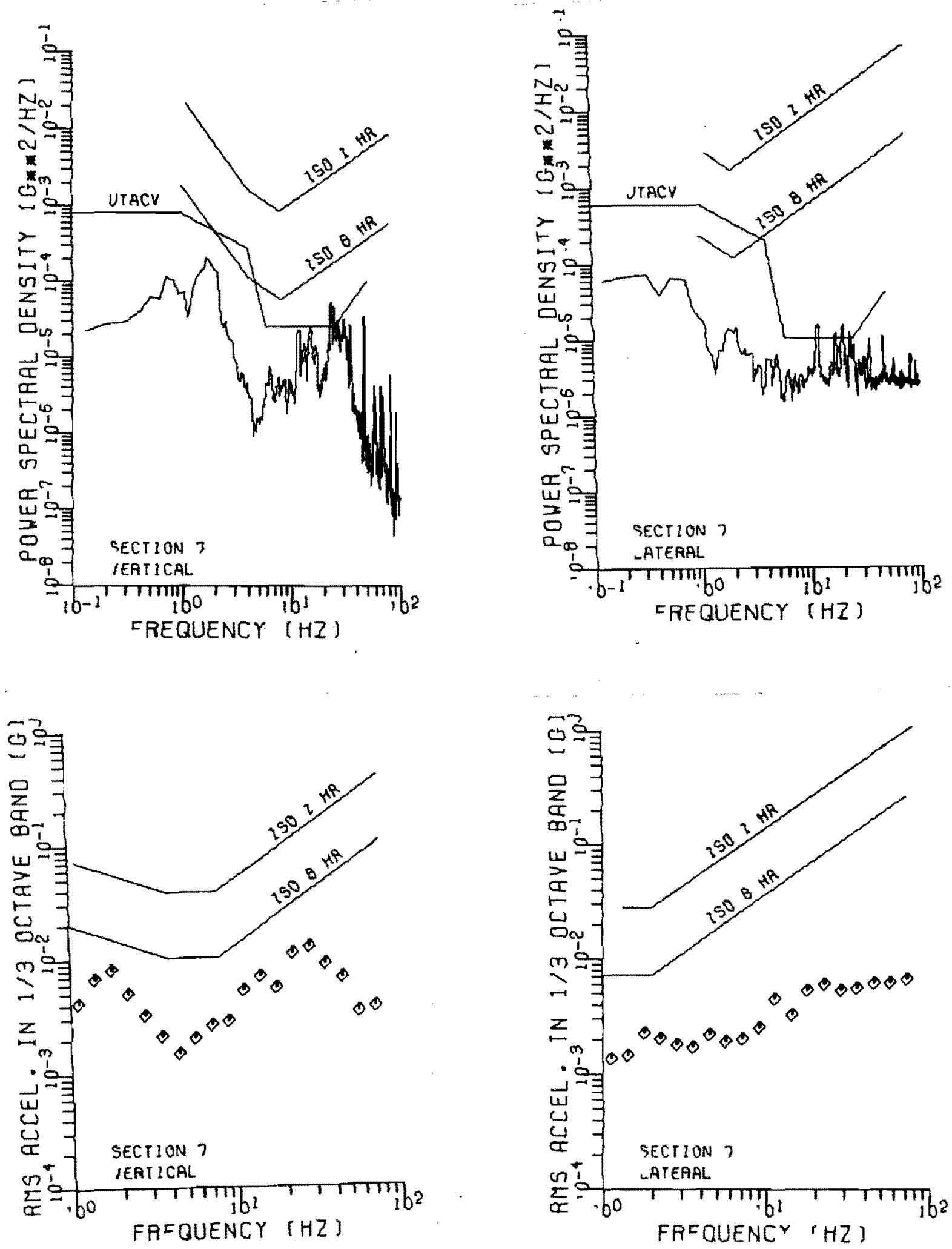


\section{BUICK FLOOR VIBRATIONS}
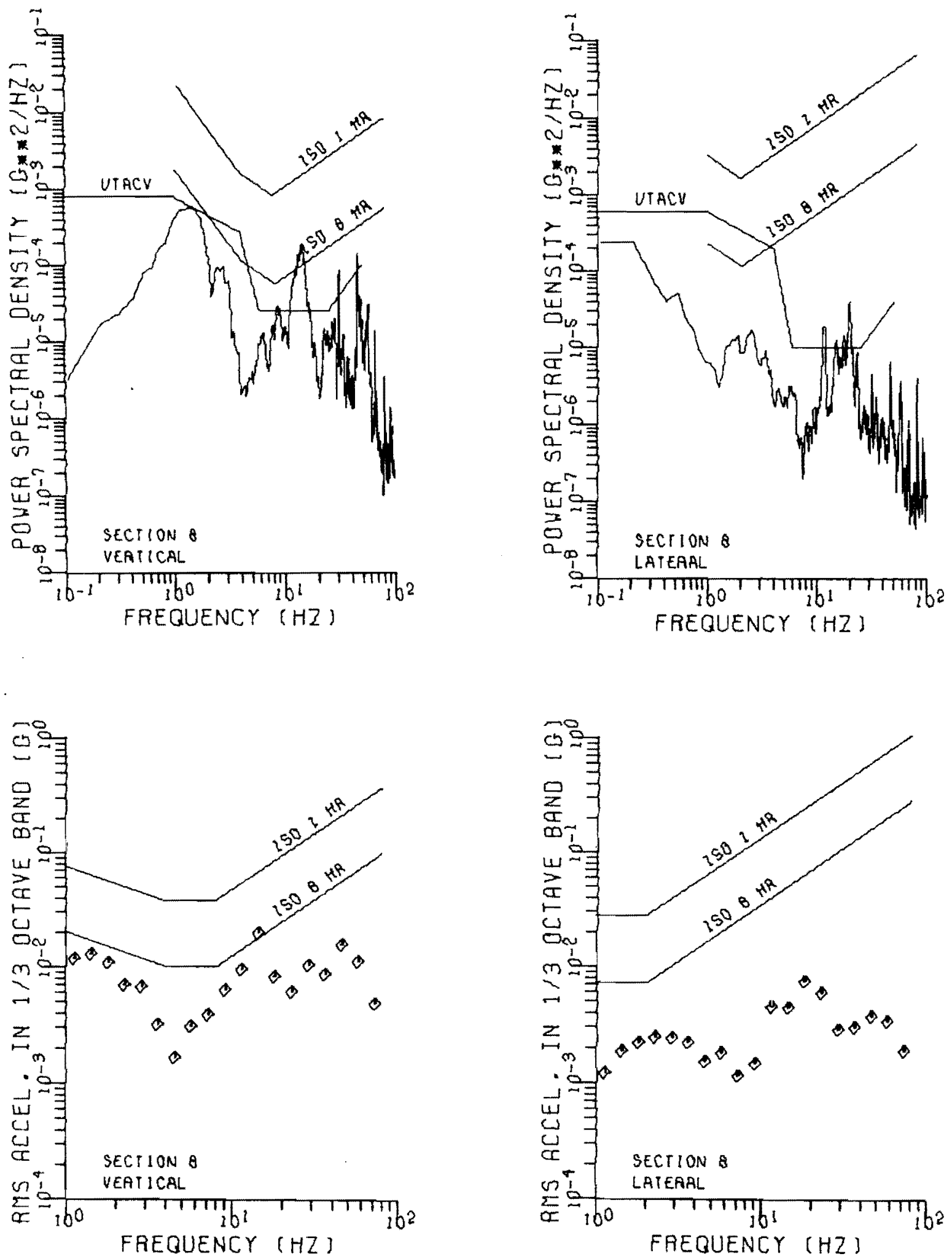


\section{BUICK FLOOR VIBRATIONS}
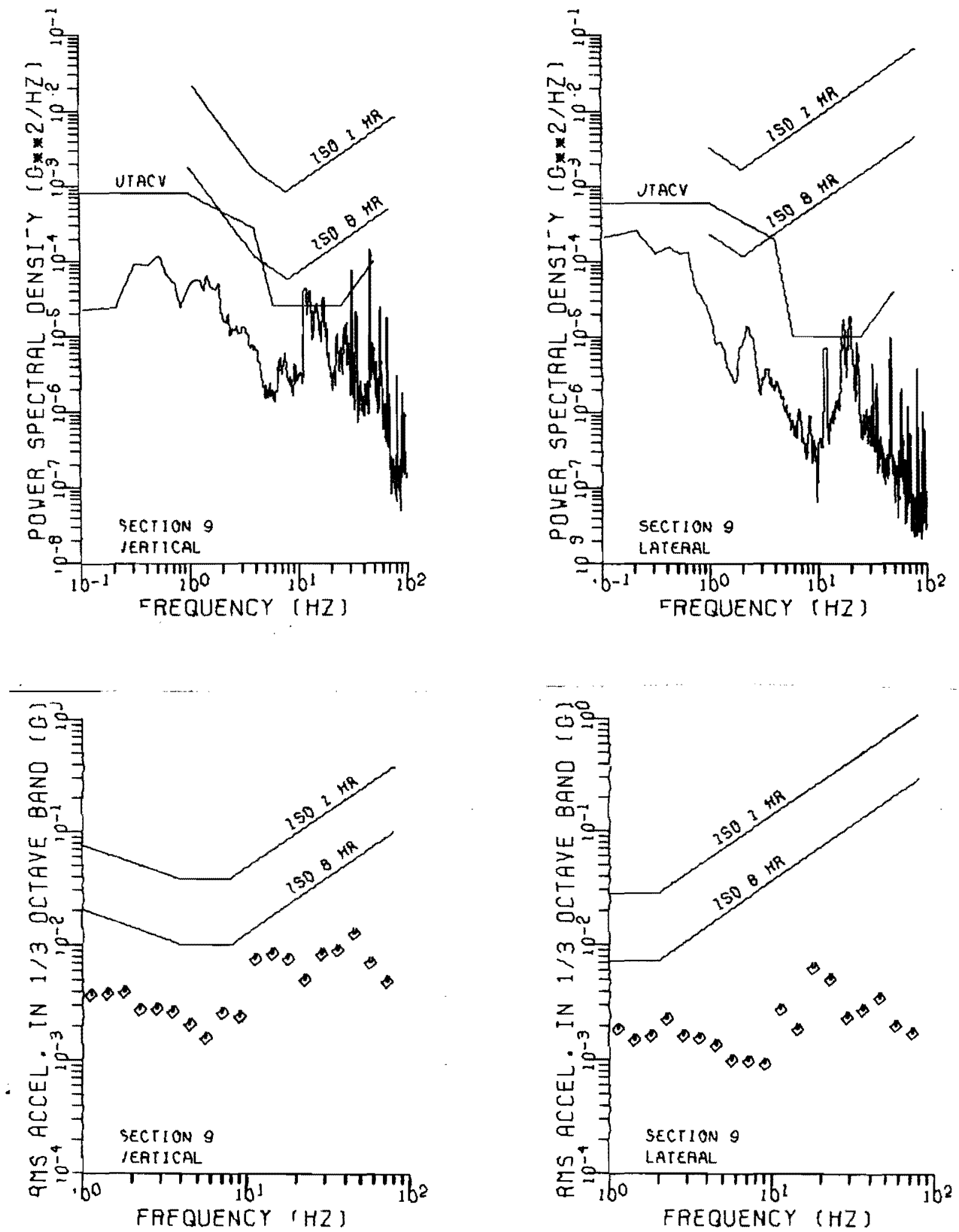


\section{BUICK FLOOR VIBRATIONS}
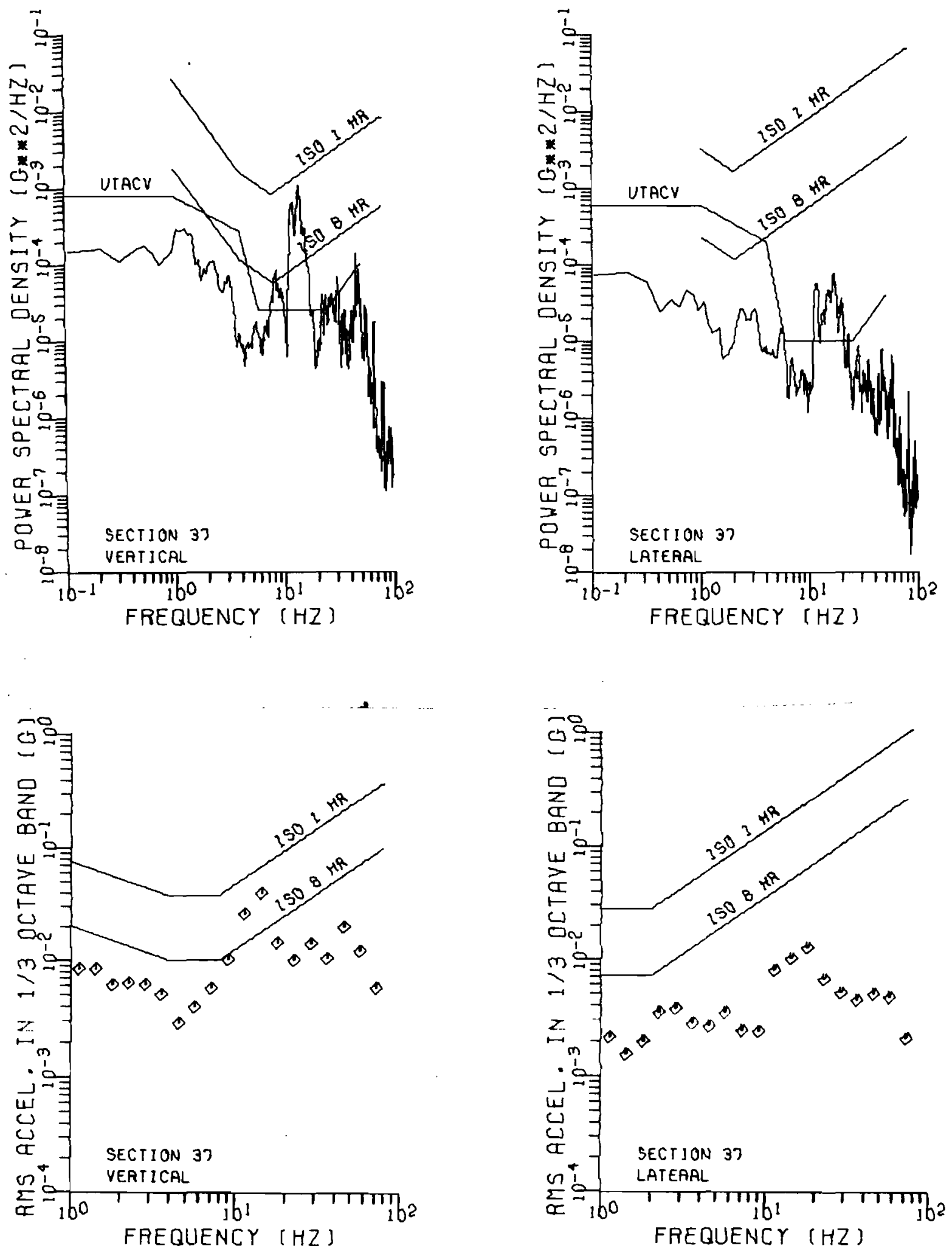


\section{BUICK FLOOR VIBRATIONS}
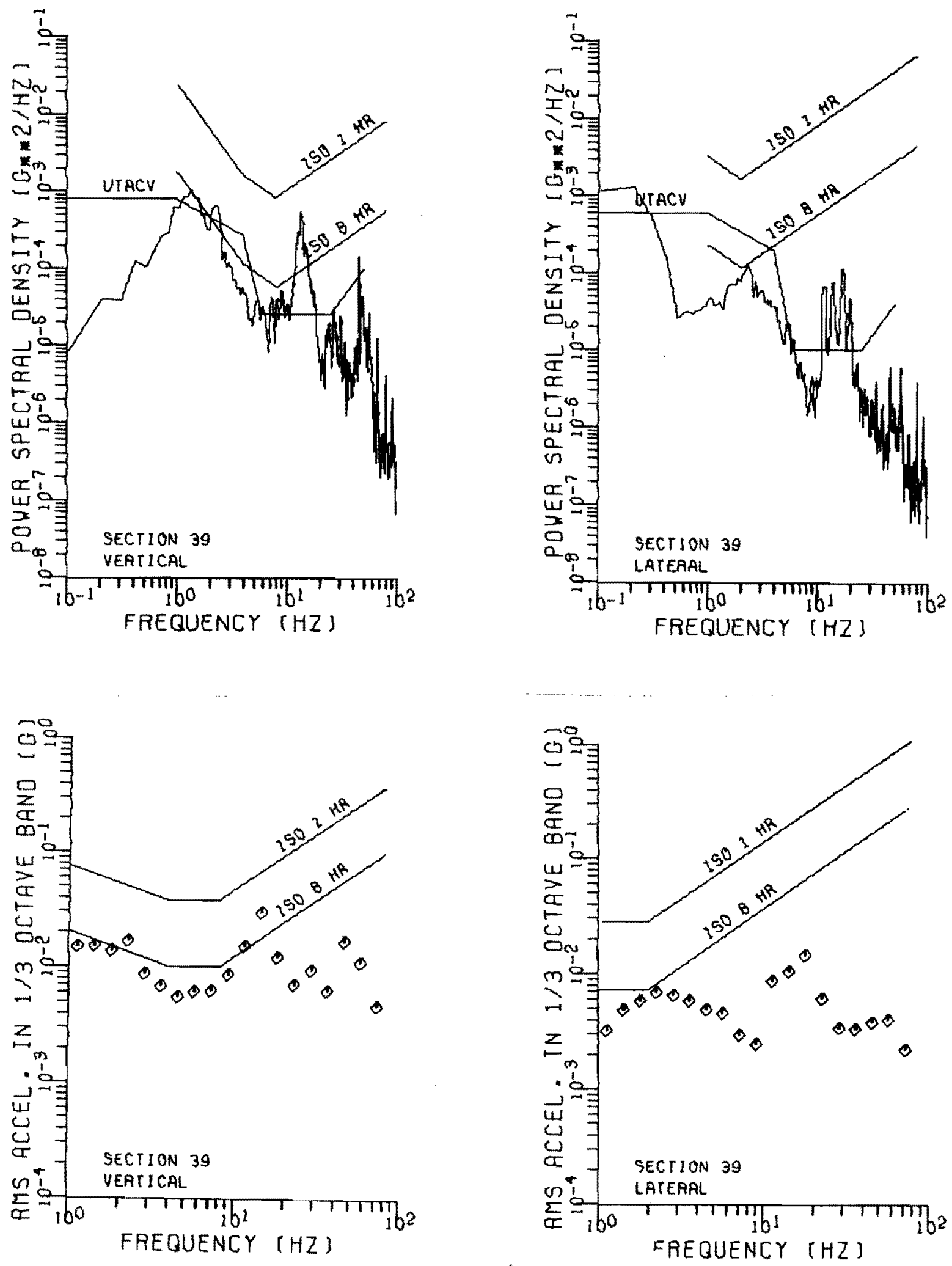

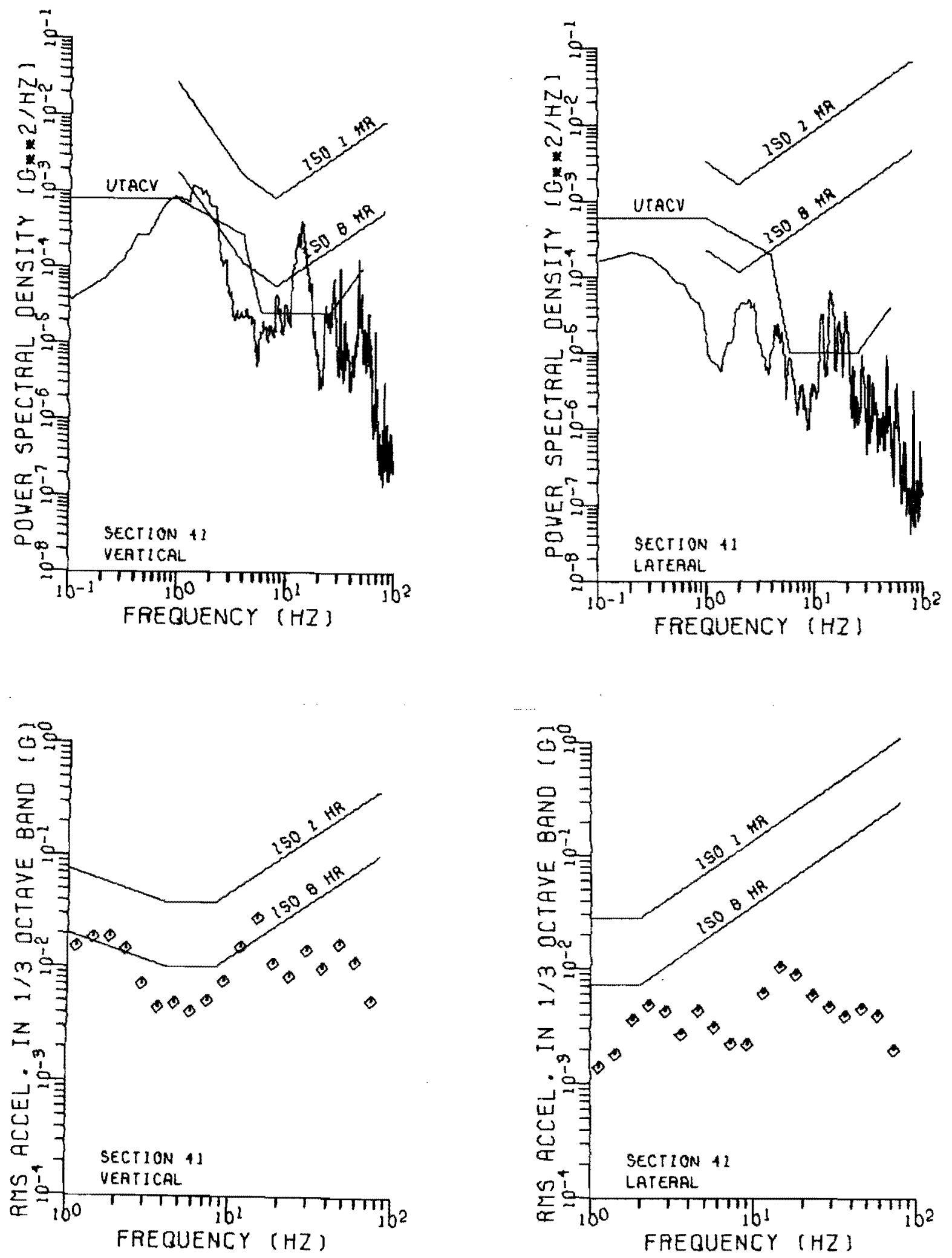


\section{MAVERICK FLOOR VIBRATIONS}
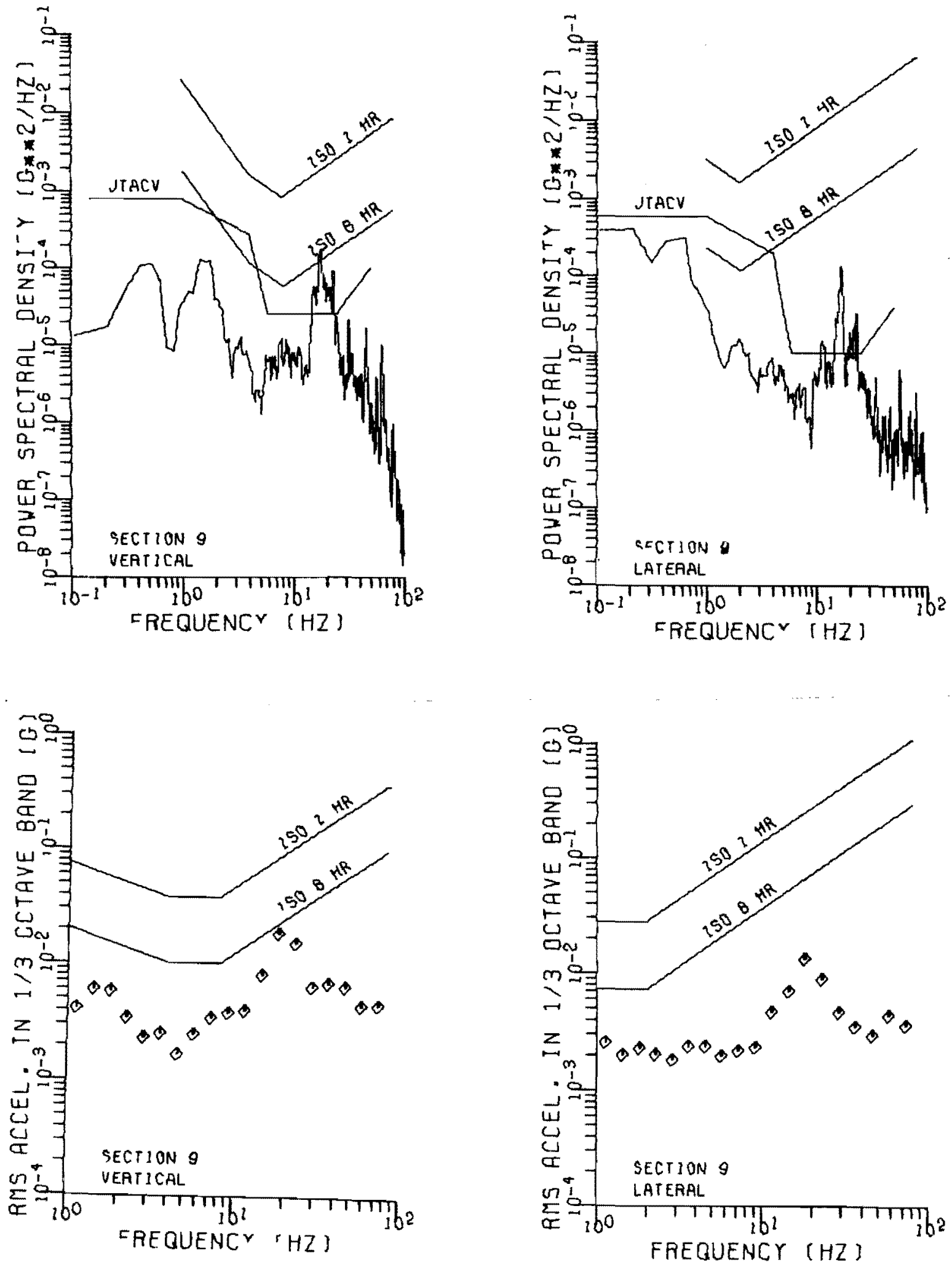
MAVERICK FLOOR VIBRATIONS
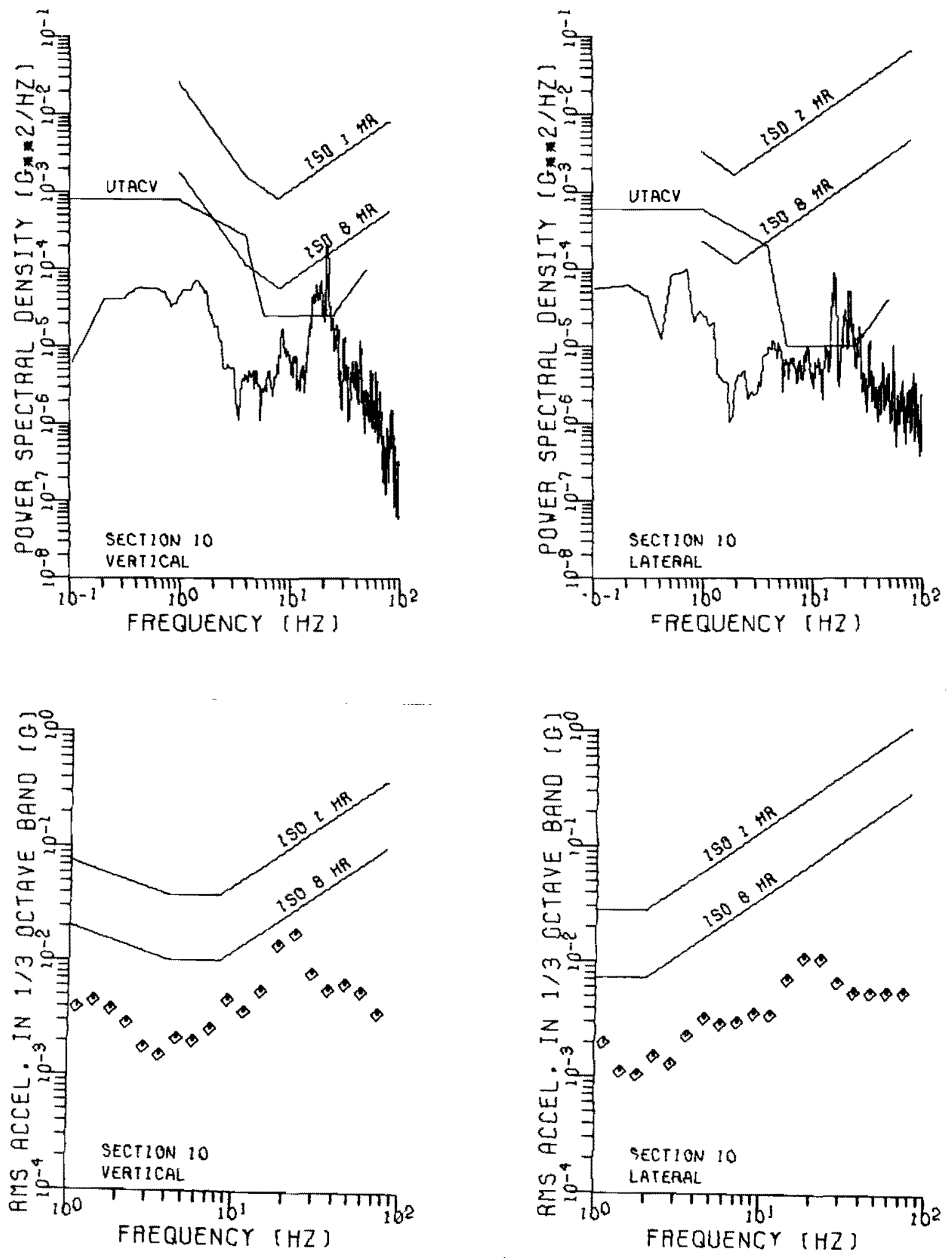

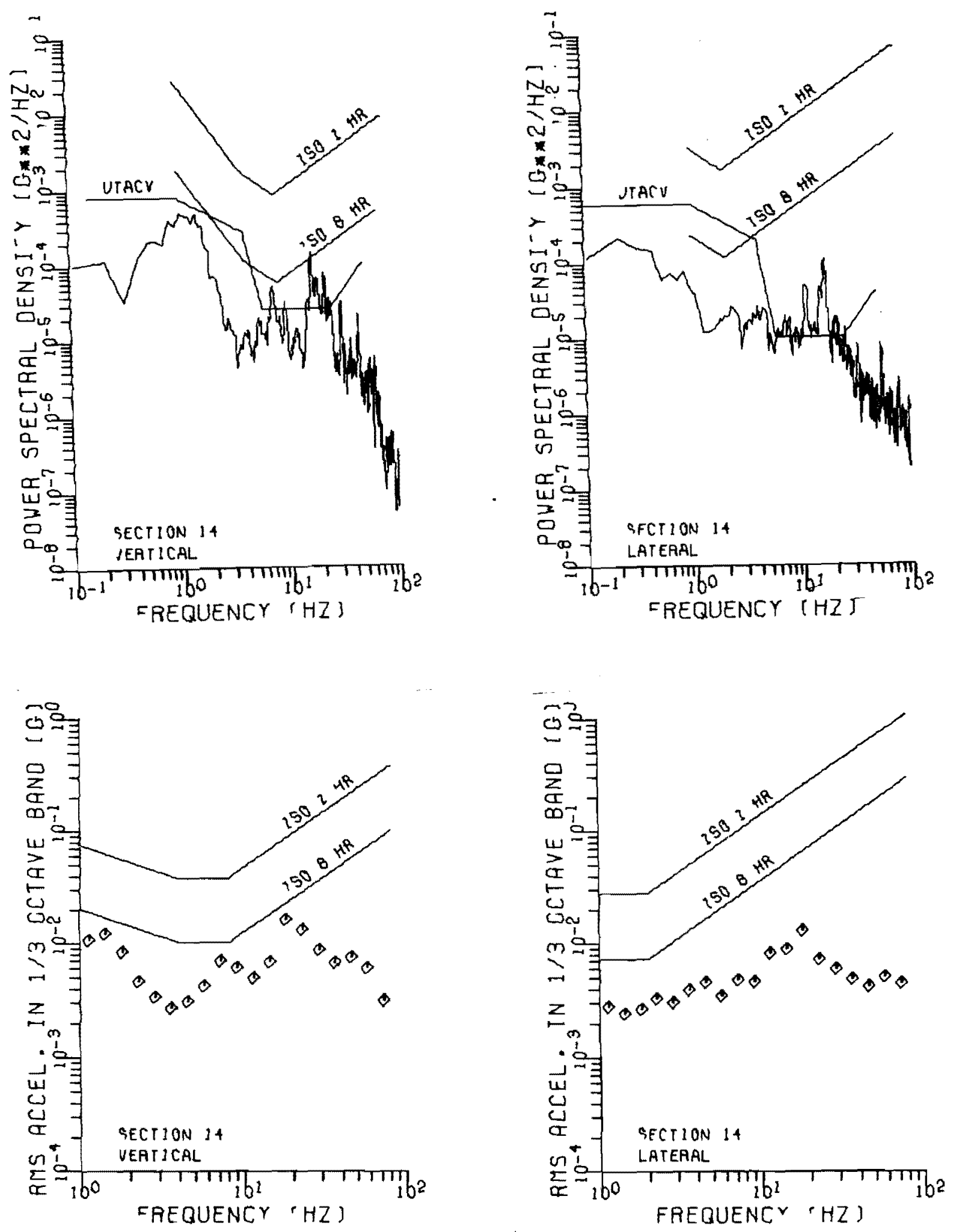

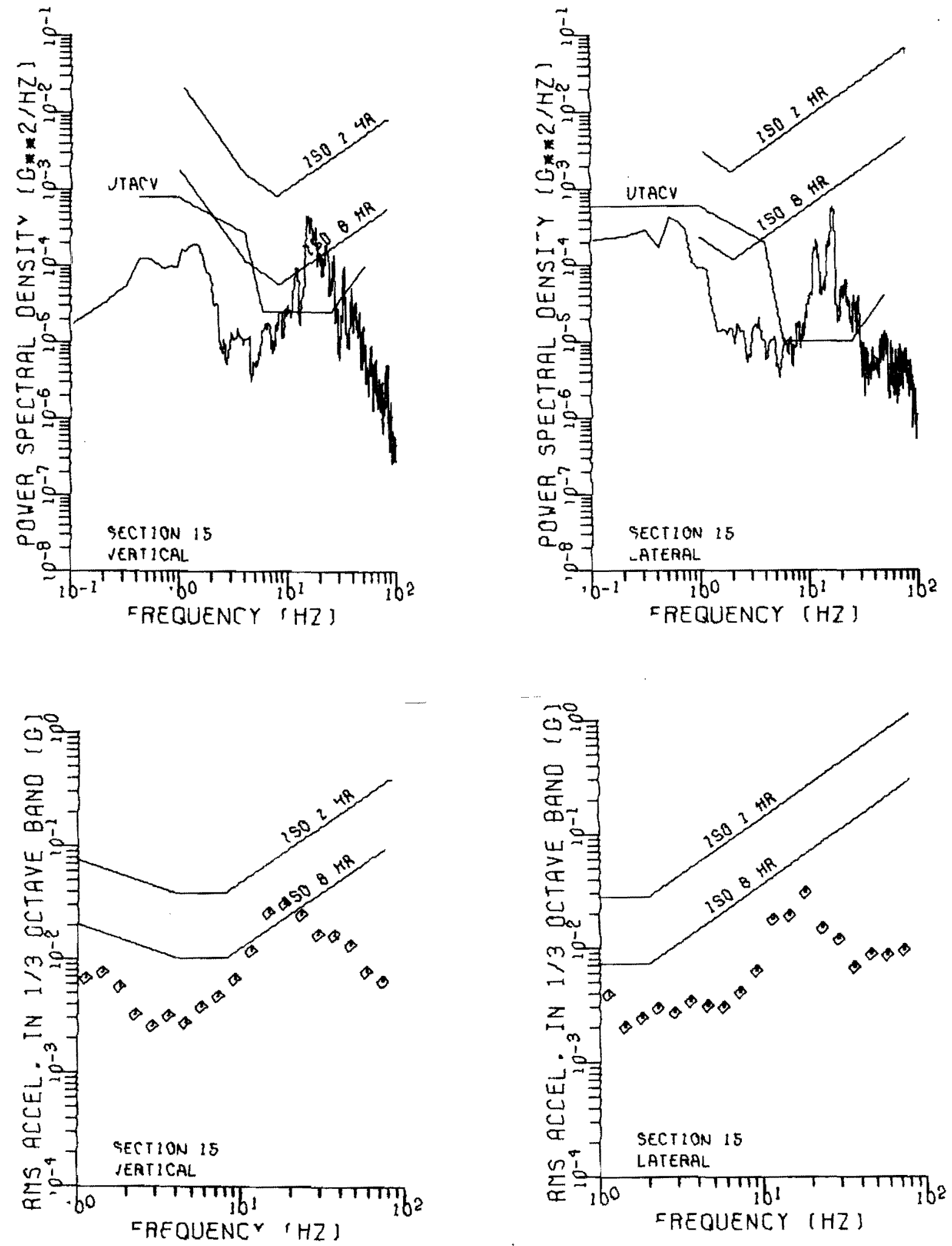
MAVERICK FLOOR VIBRATIONS
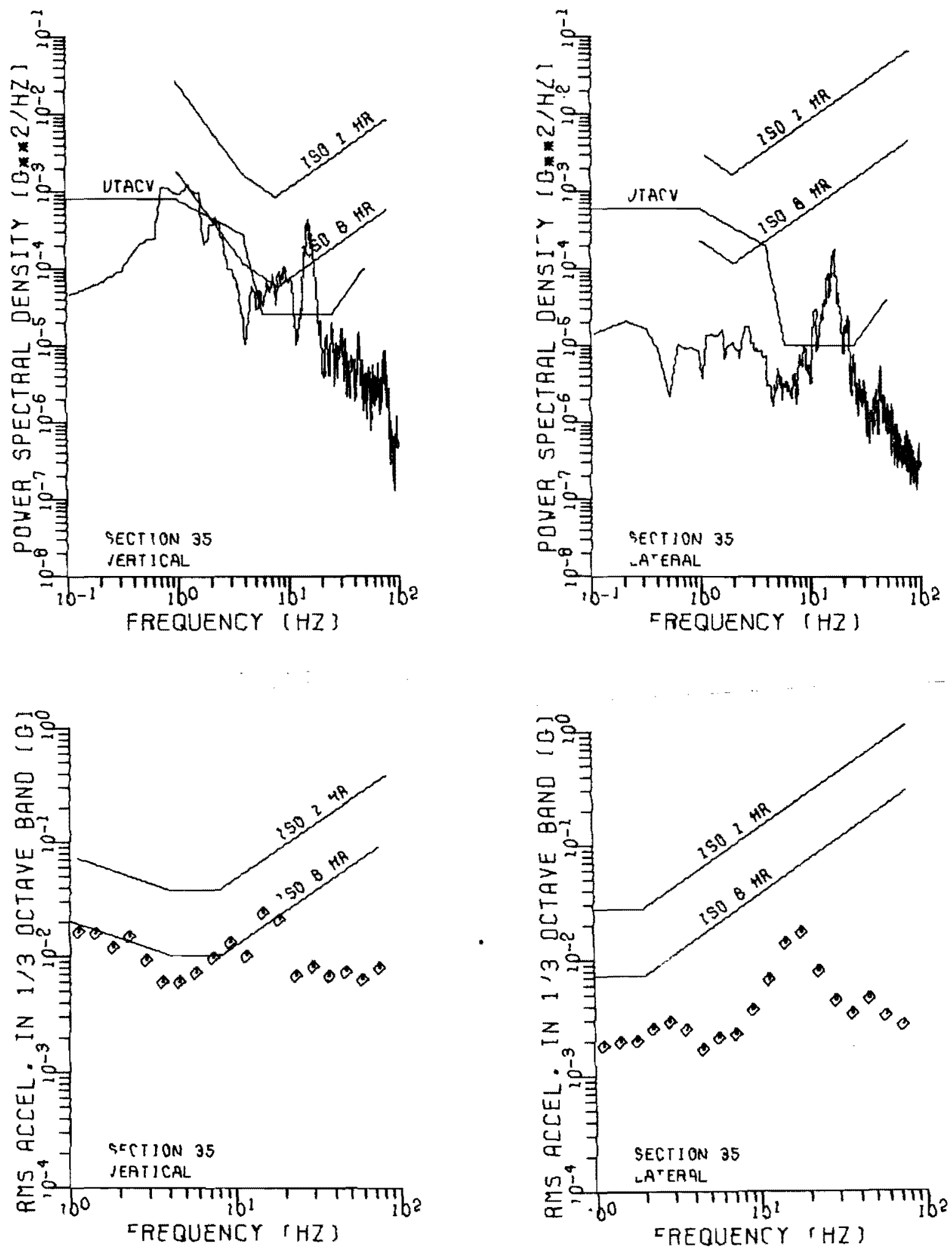
MAVERICK FLOOR VIBRATIONS
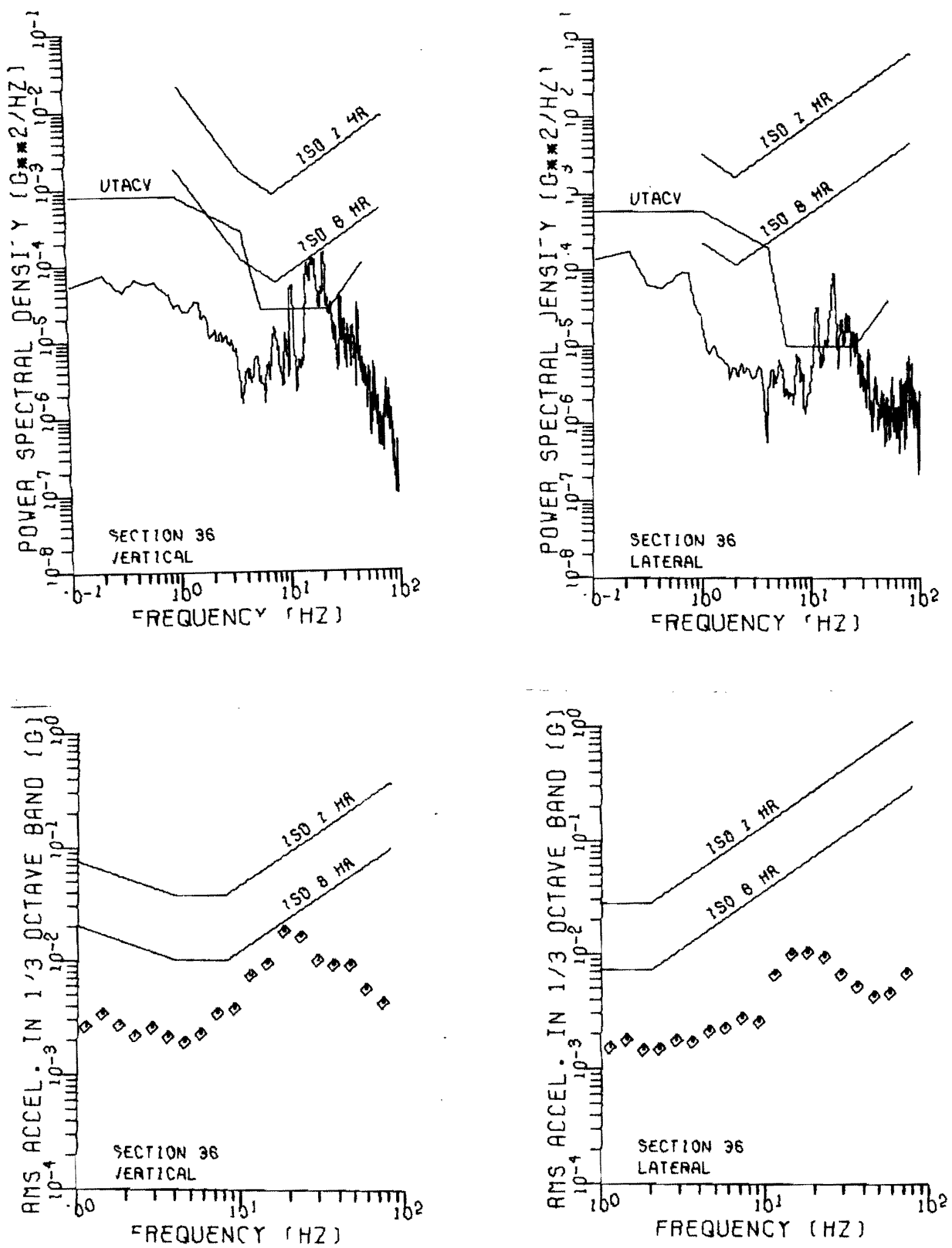

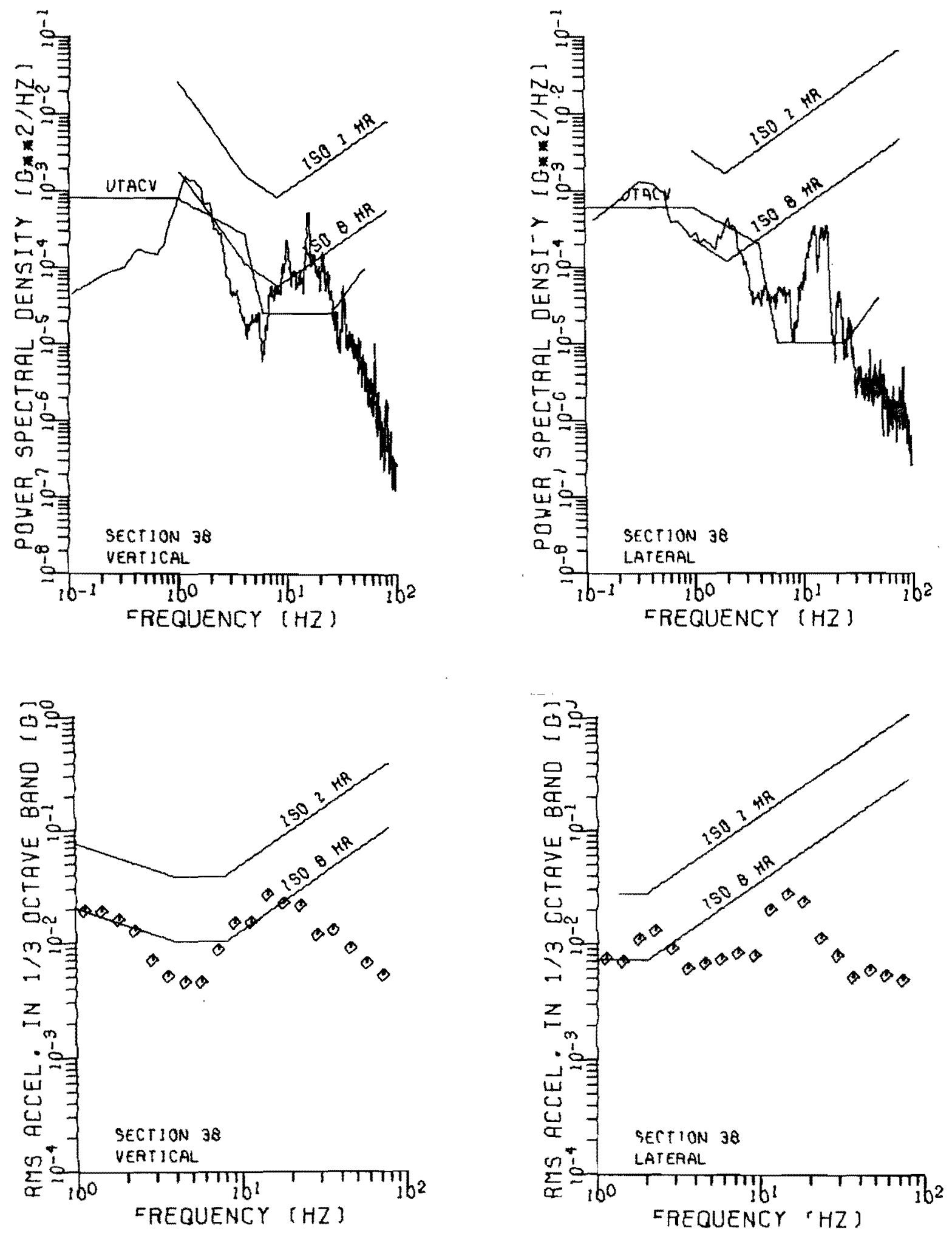

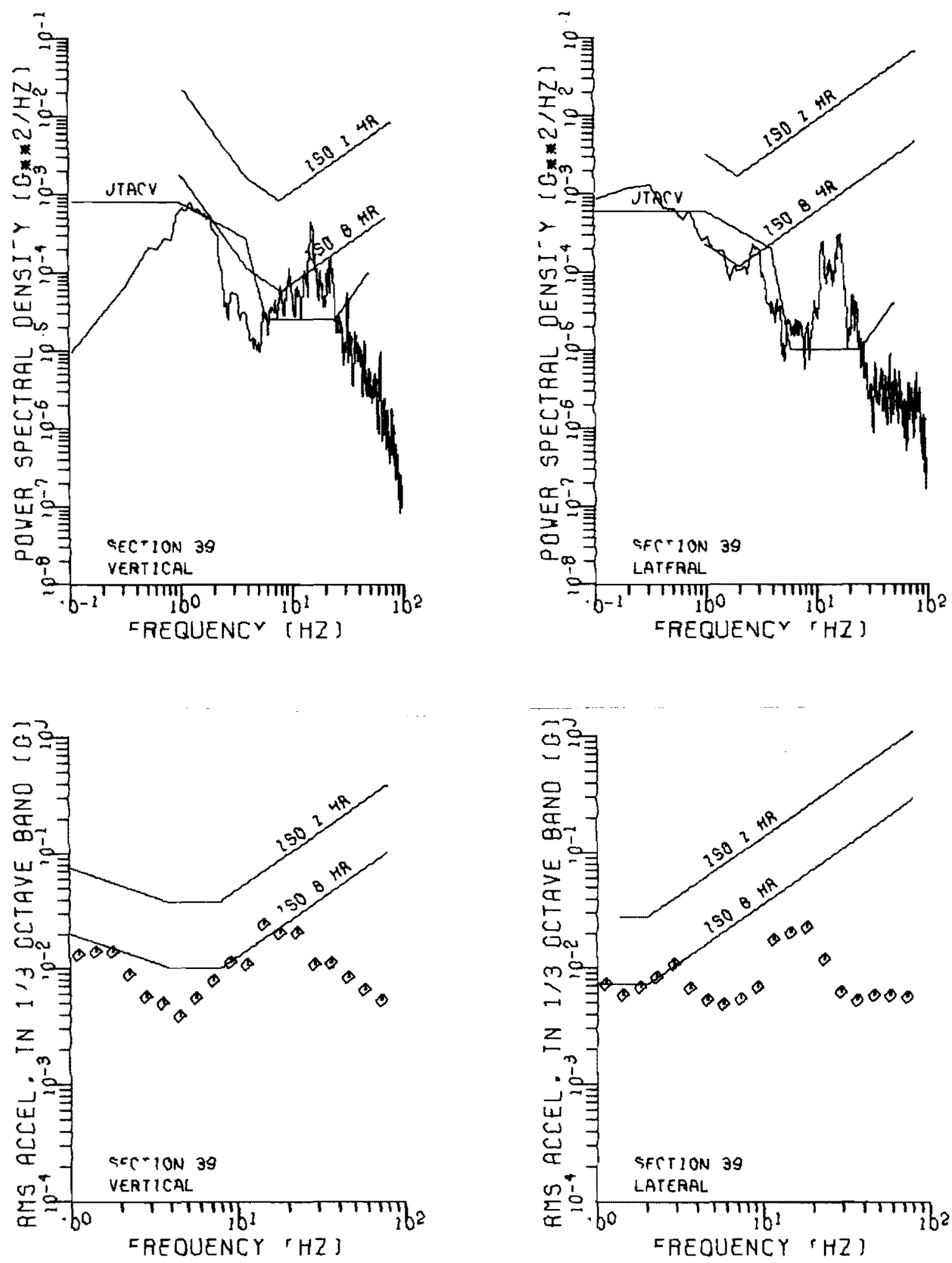

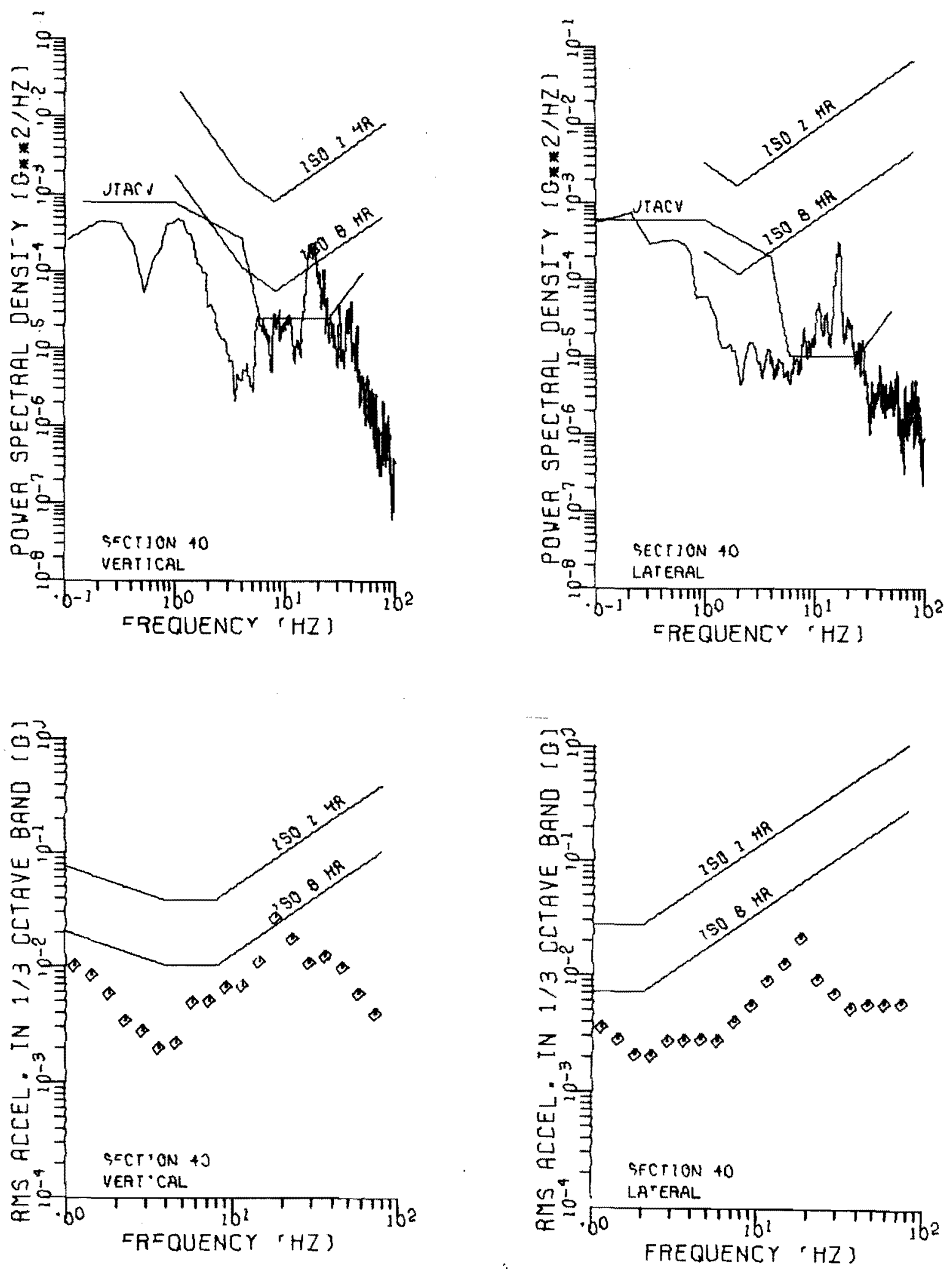

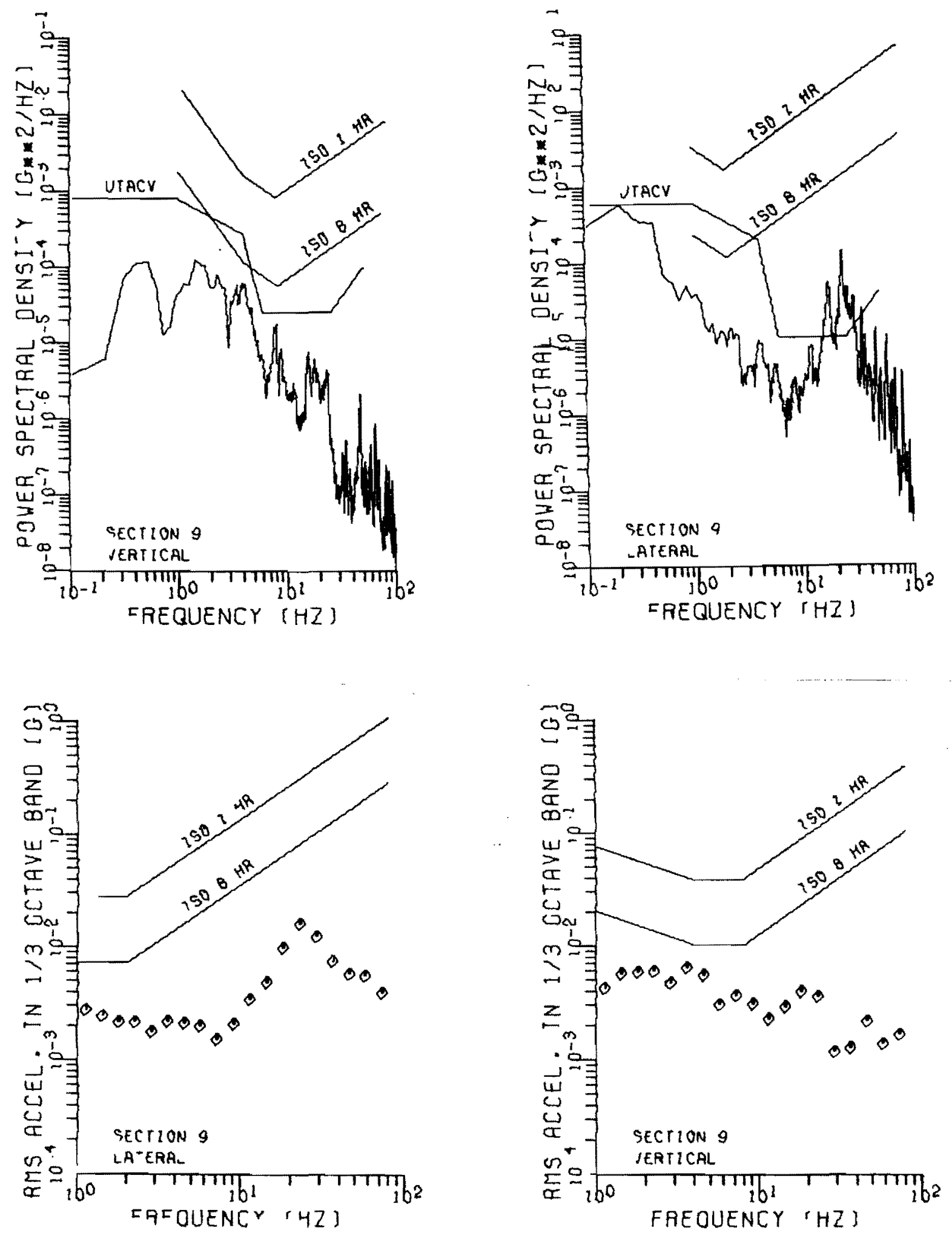
MAVERICK SEAT VIBRATIONS
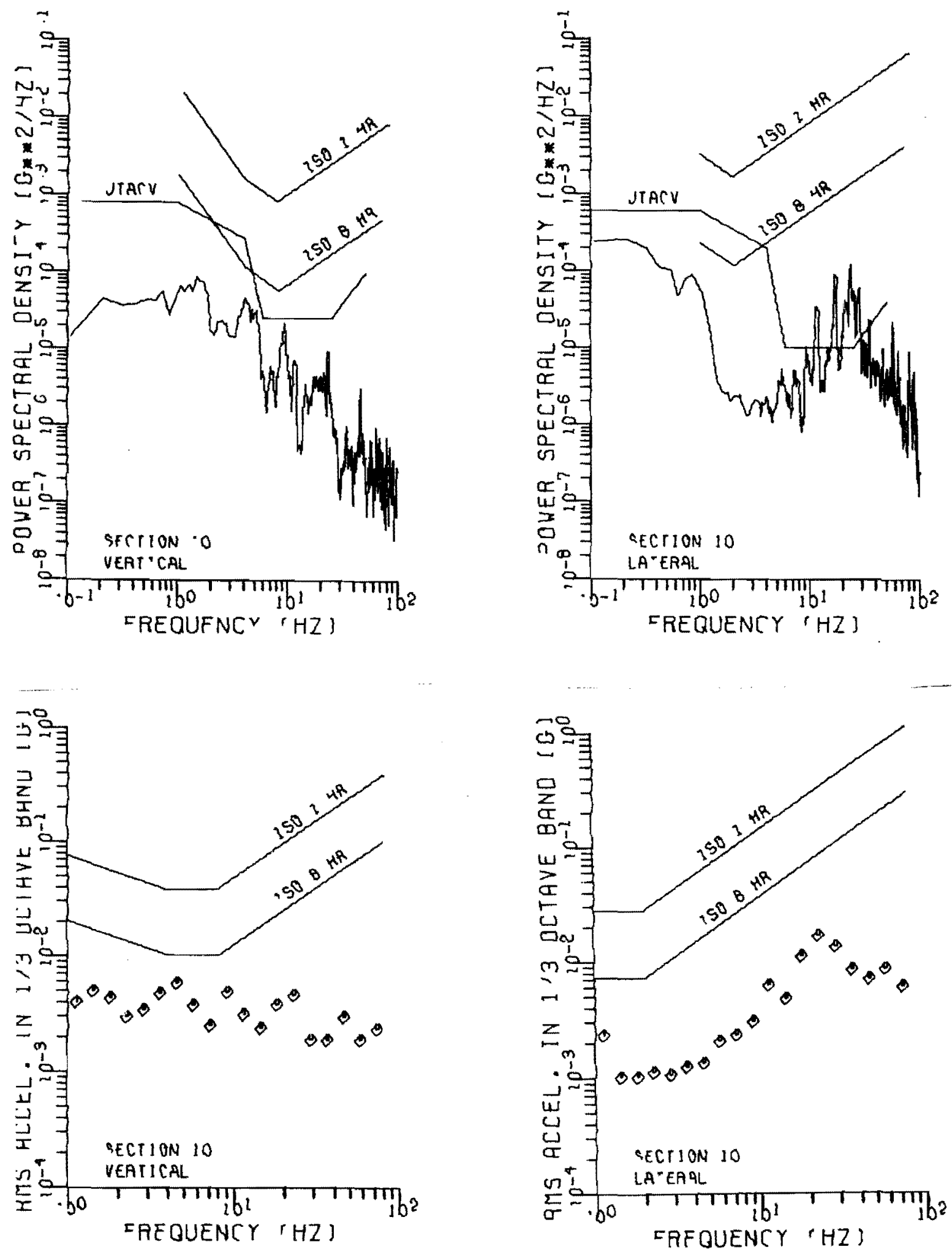
MAVERICK SEAT VIBRATIONS
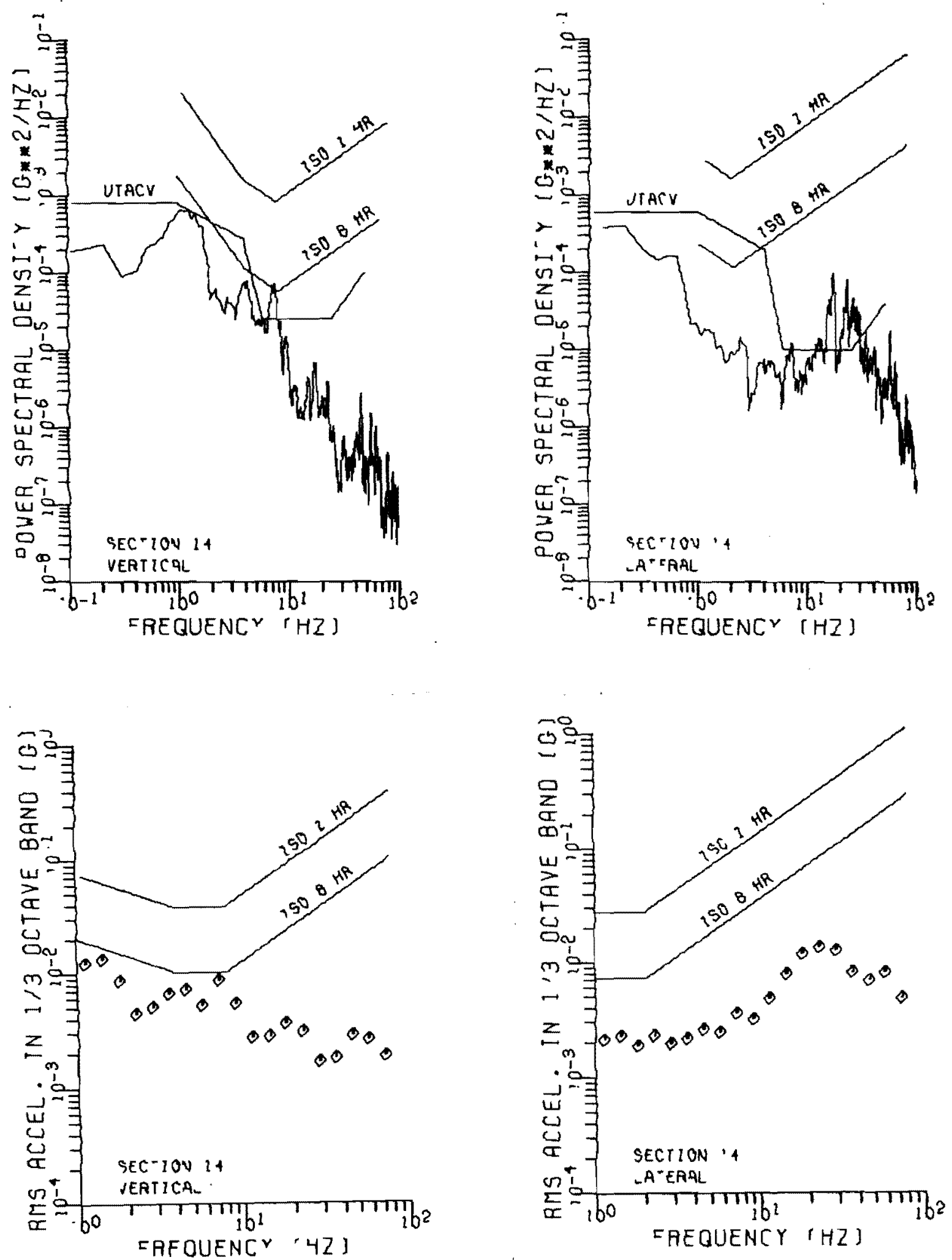
MAVERICK SEAT VIBRATIONS
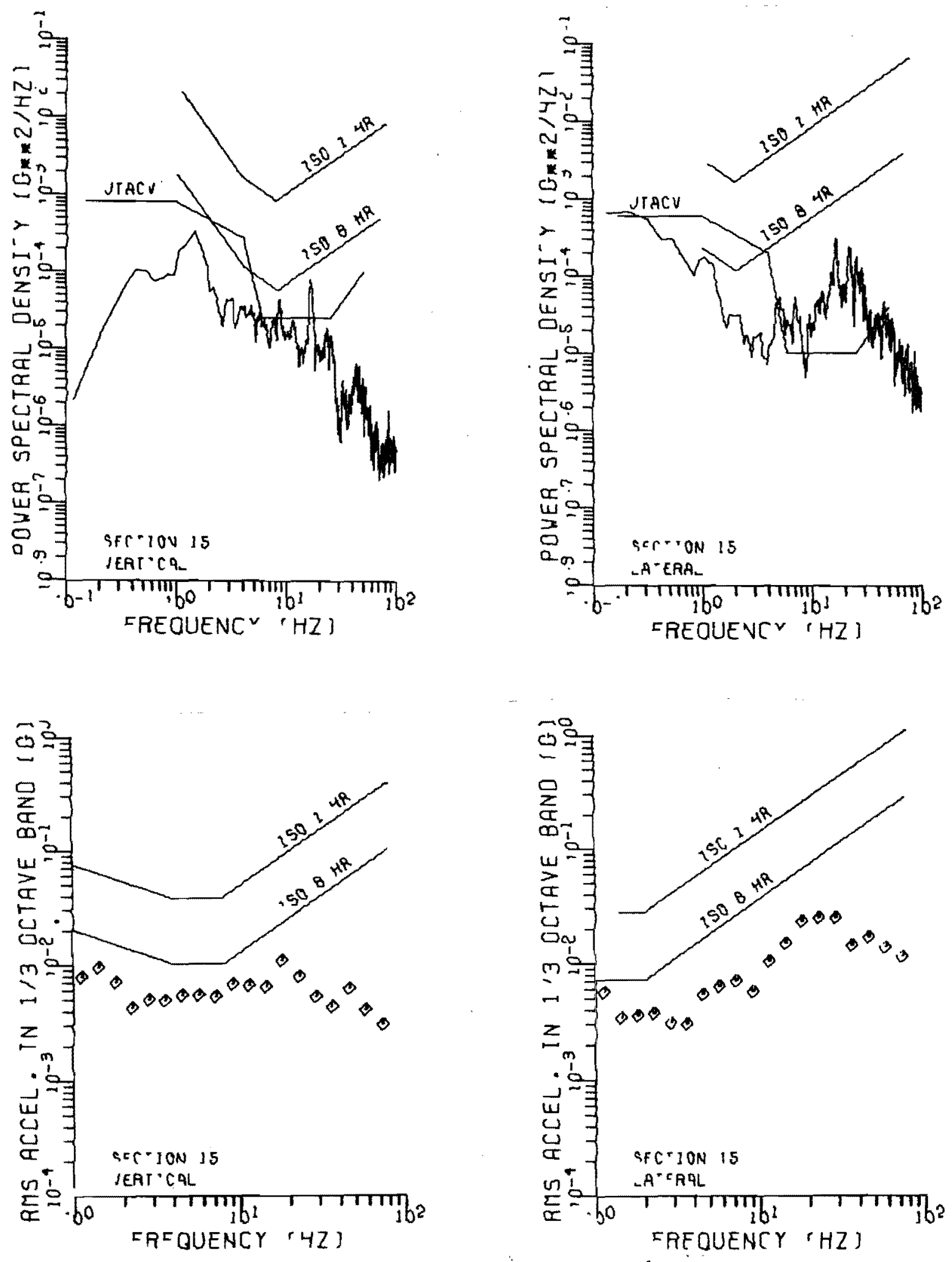

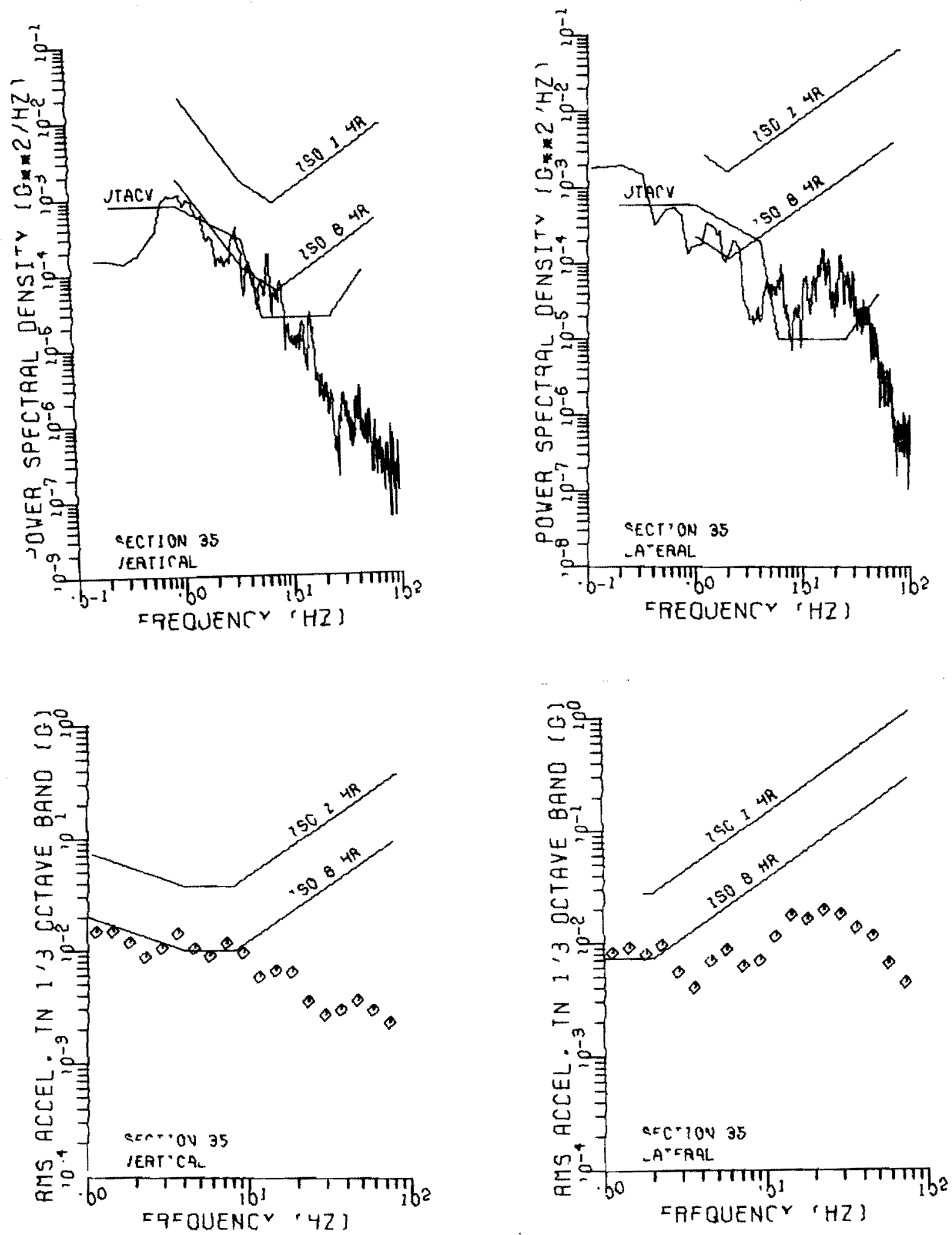

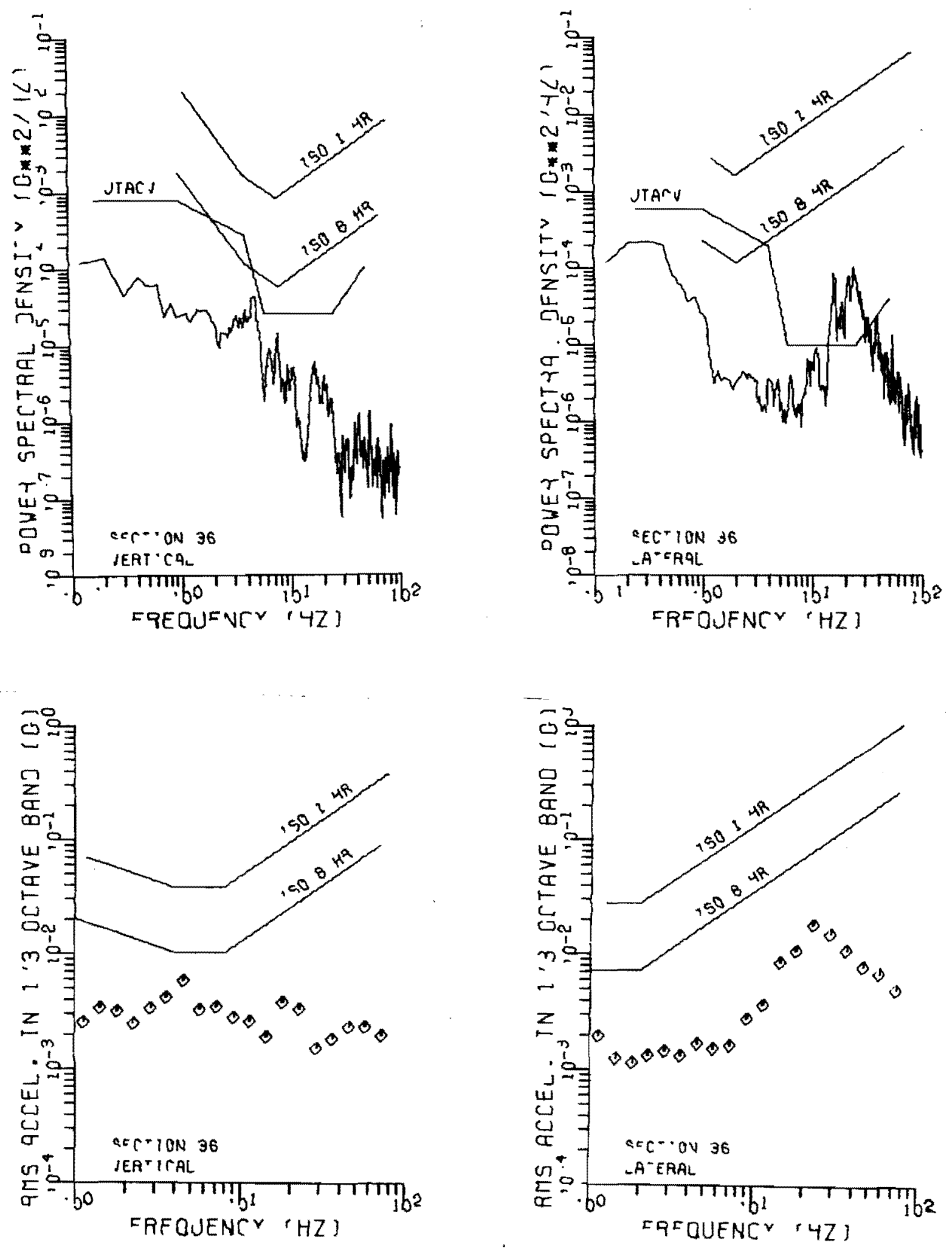

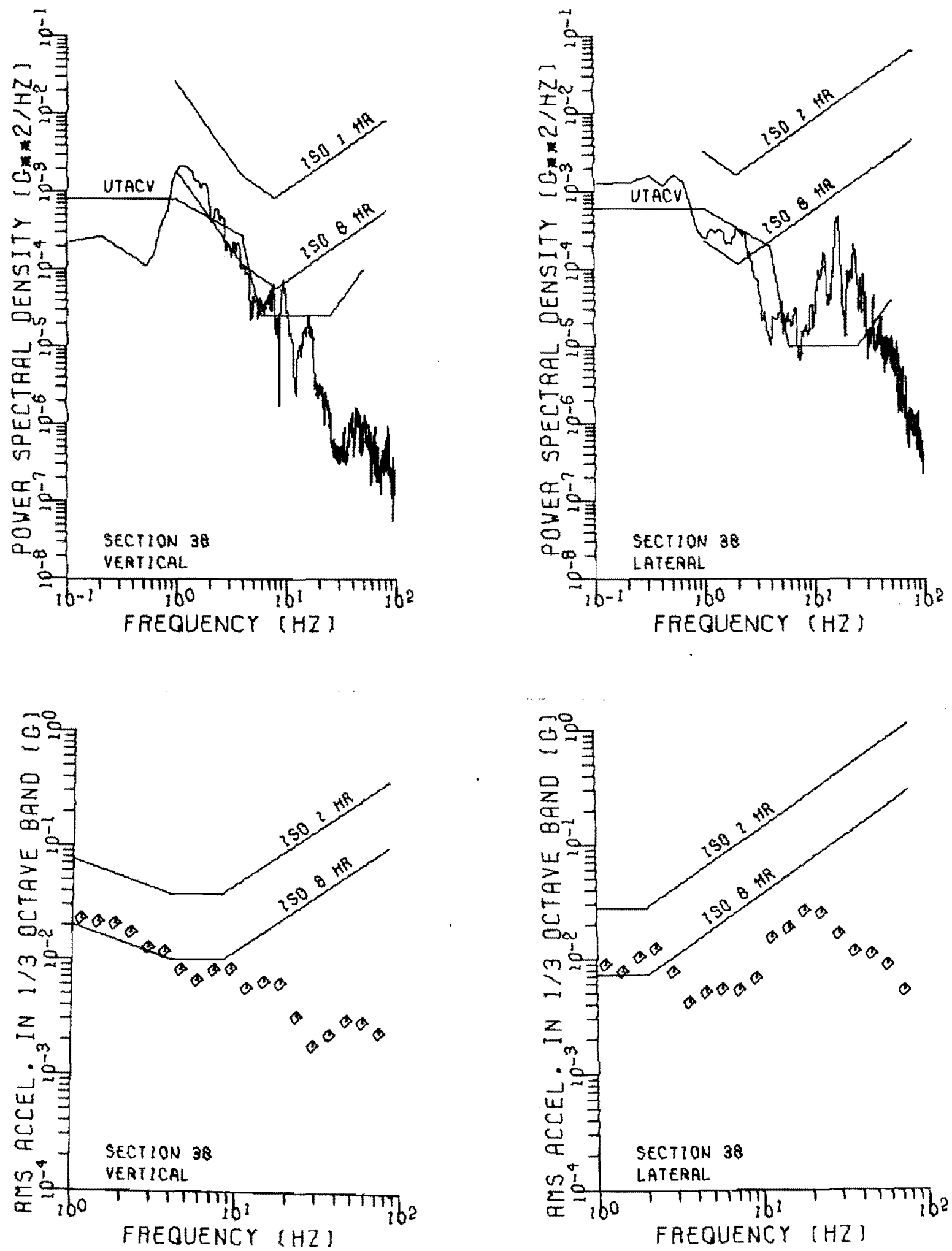
MAVERICK SEAT VIBRATIONS
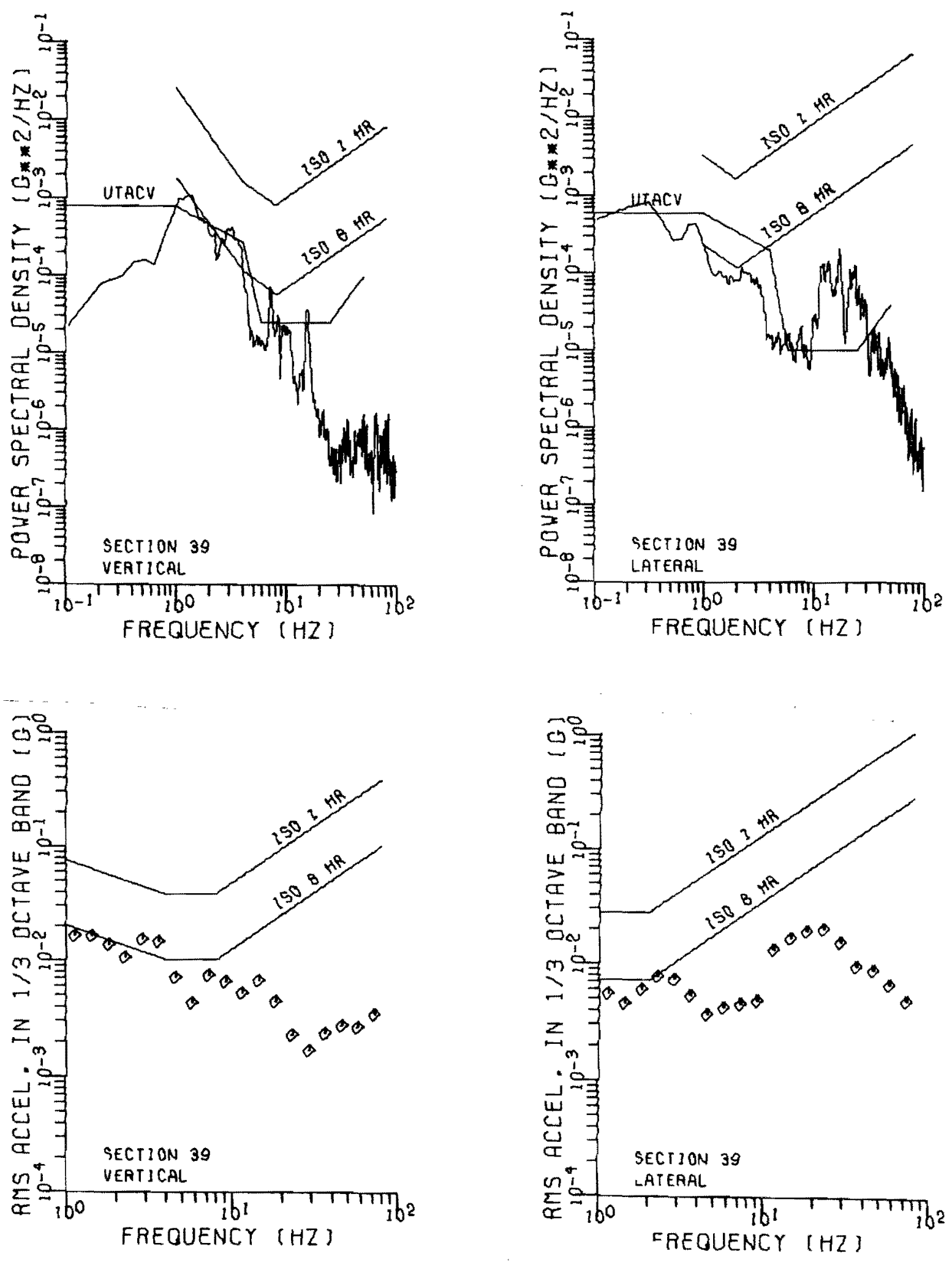

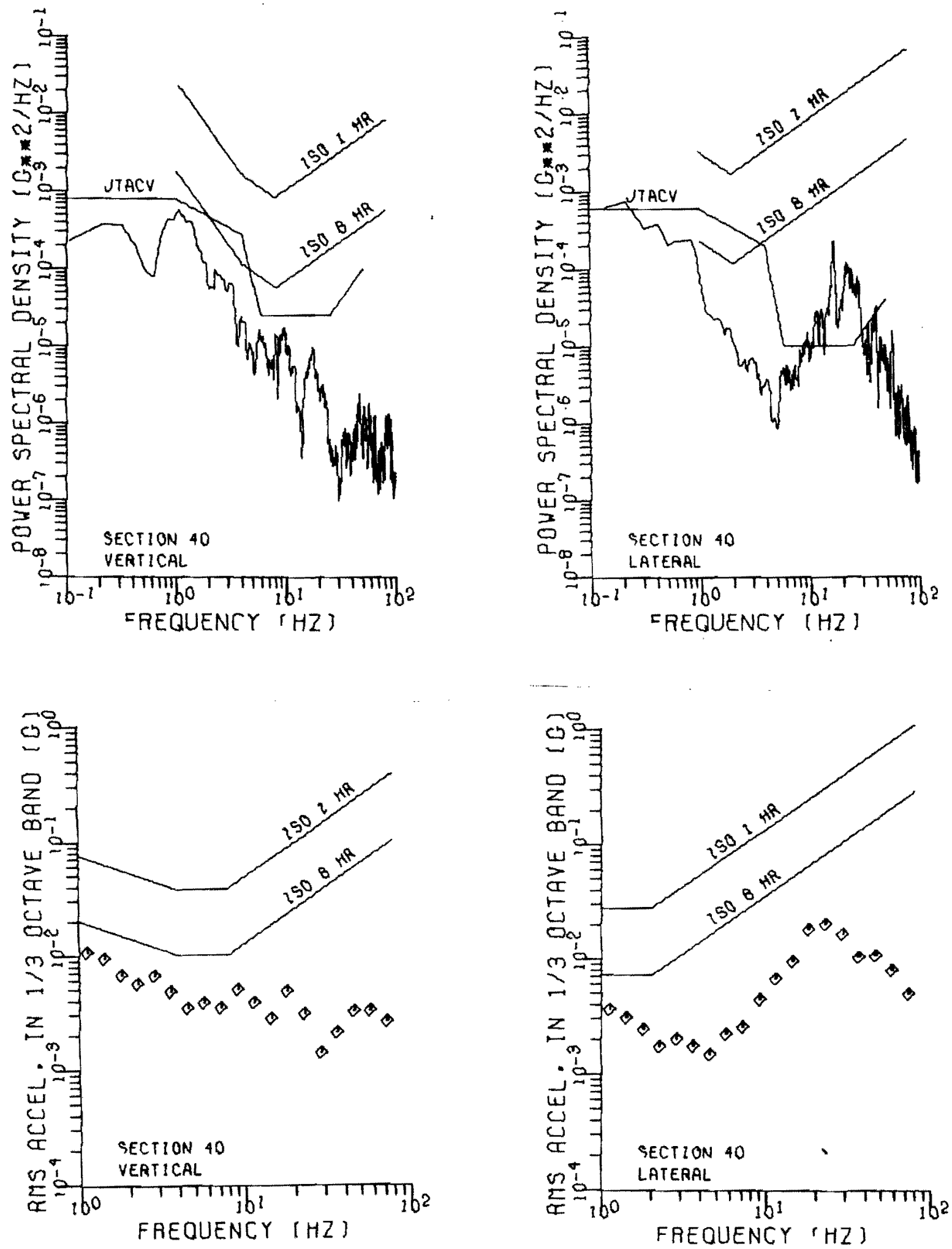
APPENDIX D

WEIGHTED INDICES 


\begin{tabular}{|c|c|c|c|c|c|c|c|c|}
\hline & & & & JICK FLOO & TRO VIBRA & & & \\
\hline JANEWAY & DIEKMAN & UTACVV & UTACVL & UTACVM & I SJV & ISOL & ISOM & RMSVI00 \\
\hline .049390 & .004067 & .036529 & .022084 & .042684 & .059114 & .062508 & .086012 & .042709 \\
\hline .072695 & .100454 & .053146 & .025753 & .059058 & .095195 & .099985 & .138056 & .065195 \\
\hline .057242 & .076848 & .042886 & $.02007 \mathrm{~B}$ & .042434 & .070994 & .063215 & .095059 & .050346 \\
\hline .034671 & .040308 & .025428 & .013605 & $.0<8436$ & .035717 & .037052 & .053590 & .027719 \\
\hline .064559 & .104904 & .046429 & .026580 & .046499 & .100203 & .067826 & .121000 & .067741 \\
\hline .072403 & .041521 & .025295 & .015654 & $.0<9747$ & .035942 & .042601 & .055799 & .027388 \\
\hline .051813 & .001991 & .037067 & .015613 & .048461 & .062628 & .049497 & .079827 & .045043 \\
\hline .032060 & .055531 & .023829 & .011877 & $.0<6024$ & .032762 & .056993 & .065733 & .027379 \\
\hline .097538 & .093805 & .069579 & .025979 & .074260 & .095500 & .046952 & .106418 & .064502 \\
\hline .073867 & .005755 & .051477 & .027492 & .058322 & .093372 & .111581 & .145494 & .058548 \\
\hline .070958 & .090334 & $.05038 \mathrm{~A}$ & .021637 & .024829 & .092871 & .063668 & .112600 & .061165 \\
\hline RMSL 100 & RMSMIOO & RMSV40 & RM\$ $\$ 40$ & RMS 440 & APIRV & APWRL & APWRT & MPR \\
\hline $0>3193$ & .048607 & .041903 & .022424 & .047532 & .043480 & .049080 & .092560 & 3.500000 \\
\hline .070406 & .071983 & .065026 & .029764 & .071517 & .114180 & .140798 & .254974 & 2.330000 \\
\hline$. n>1807$ & .054801 & .047235 & .020761 & $.0>1591$ & .063430 & .055360 & .1188800 & 3.830000 \\
\hline .014425 & .031254 & .026389 & .013569 & .029670 & .021380 & .023680 & .045040 & 4.420000 \\
\hline$=n>6573$ & .072761 & .065365 & .026191 & .070414 & .103900 & .073200 & .177100 & 3.000000 \\
\hline .070605 & .034648 & .026714 & .015938 & .031113 & .018200 & .029920 & .048120 & 4.250000 \\
\hline .017395 & .048281 & .039471 & .016546 & .042794 & .056060 & .018680 & .074720 & 3.500000 \\
\hline .015839 & .0 .31636 & .023603 & .014835 & $.0<7874$ & .016680 & .035640 & .052320 & 4.170000 \\
\hline .074607 & .069028 & .061108 & .023914 & .065620 & .152382 & .031240 & .183620 & 2.230000 \\
\hline .032951 & .067232 & .054009 & .033022 & .063300 & .137760 & .087260 & .225040 & 2.330000 \\
\hline .023292 & .065450 & .056554 & .022670 & .060924 & .120620 & .048614 & .169246 & 3.000000 \\
\hline
\end{tabular}




\begin{tabular}{|c|c|c|c|c|c|c|c|c|}
\hline \multirow[b]{2}{*}{ JANEWAY } & \multirow[b]{2}{*}{ DIEKKMAN } & \multicolumn{6}{|c|}{ MAVERICK FLOORBOARD VIGRATIONS } & \multirow[b]{2}{*}{ RMSV100 } \\
\hline & & UTACVV & UTACVL & UTACVM & ISOV & I SOL & I SOM & \\
\hline .047344 & .048536 & .034603 & .023834 & .042016 & .041079 & .077258 & .087497 & .032272 \\
\hline .042725 & .044237 & .032032 & .024254 & .040150 & .035785 & .043968 & .056696 & .029826 \\
\hline .046157 & .006908 & .033602 & .026313 & .042984 & .054645 & .061702 & .082420 & .037689 \\
\hline .089548 & .088483 & .065704 & .055212 & .005614 & .073412 & .090736 & .116715 & $.05888 \mathrm{~B}$ \\
\hline .071954 & .088408 & .049926 & .030913 & .058721 & .094950 & .033616 & .100720 & .057742 \\
\hline .051204 & .051421 & .037977 & .024763 & .045340 & .041861 & .050063 & .065252 & .035016 \\
\hline .085621 & .049843 & .061313 & .057527 & .004075 & .099893 & .169140 & .196434 & .064751 \\
\hline .076502 & .085800 & .055241 & .048865 & .073751 & .084027 & .159594 & .180369 & .055946 \\
\hline .065436 & .071587 & .052199 & .037870 & .064488 & .061476 & .094045 & .112355 & .047277 \\
\hline RMSLIOO & RMSM100 & RMS 40 & RMSL40 & RMSM40 & AP WRV & APWRL & APWRT & MPR \\
\hline .035824 & .041337 & .031268 & .024183 & .039499 & .025676 & .072268 & .097940 & 4.090000 \\
\hline .024466 & .038608 & .028157 & .022345 & .015963 & .019360 & .035440 & .054800 & 4.070000 \\
\hline - 027011 & .046386 & .037816 & .025597 & .045679 & .039740 & .049856 & .129340 & 3.850000 \\
\hline .050954 & .077881 & .055904 & .047815 & .073567 & .086880 & .134710 & .221580 & 2.720000 \\
\hline .076729 & .003640 & .055640 & .026743 & $.001 / 45$ & .131460 & .015436 & .146900 & 2.540000 \\
\hline .075173 & .043105 & .033291 & .022783 & .040305 & .028000 & .034894 & .062900 & 3.800000 \\
\hline .058973 & .087611 & .062466 & .058520 & .005002 & .141600 & .439008 & .594868 & 2.200000 \\
\hline . 052750 & .076863 & .054645 & .052184 & .015561 & .104280 & .357240 & .461540 & 2.670000 \\
\hline .037760 & .000500 & .045227 & .036190 & .057426 & .051580 & .102940 & .154500 & 3.390000 \\
\hline
\end{tabular}




\begin{tabular}{|c|c|}
\hline $\begin{array}{l}\text { JANEWAY } \\
.049390 \\
.072695 \\
.057242 \\
074671 \\
044559 \\
032403 \\
051813 \\
.072060 \\
097538 \\
073867 \\
070958 \\
.047344 \\
.042725 \\
.046157 \\
.089548 \\
.071954 \\
041204 \\
045621 \\
.076502 \\
065436\end{array}$ & $\begin{array}{l}\text { OILKMAN } \\
.064067 \\
.100454 \\
.016848 \\
.040308 \\
.104904 \\
.041521 \\
.001991 \\
.035531 \\
.043805 \\
.085755 \\
.040334 \\
.048536 \\
.044237 \\
.056908 \\
.048483 \\
.048408 \\
.051421 \\
.049843 \\
.085800 \\
.071587\end{array}$ \\
\hline 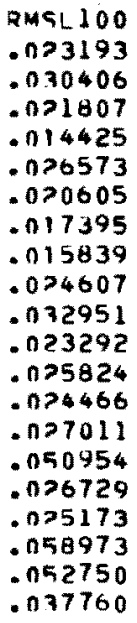 & $\begin{array}{l}\text { RMS4100 } \\
.048607 \\
.071983 \\
.054801 \\
.031254 \\
.072761 \\
.054648 \\
.048281 \\
.031636 \\
.00902 A \\
.067232 \\
.065450 \\
.041337 \\
.038604 \\
.046386 \\
.077481 \\
.063640 \\
.043105 \\
.067611 \\
.076863 \\
.060500\end{array}$ \\
\hline
\end{tabular}

MAVERTCK AVD BJICK FLOOKDUAKD VIBRATIOVS

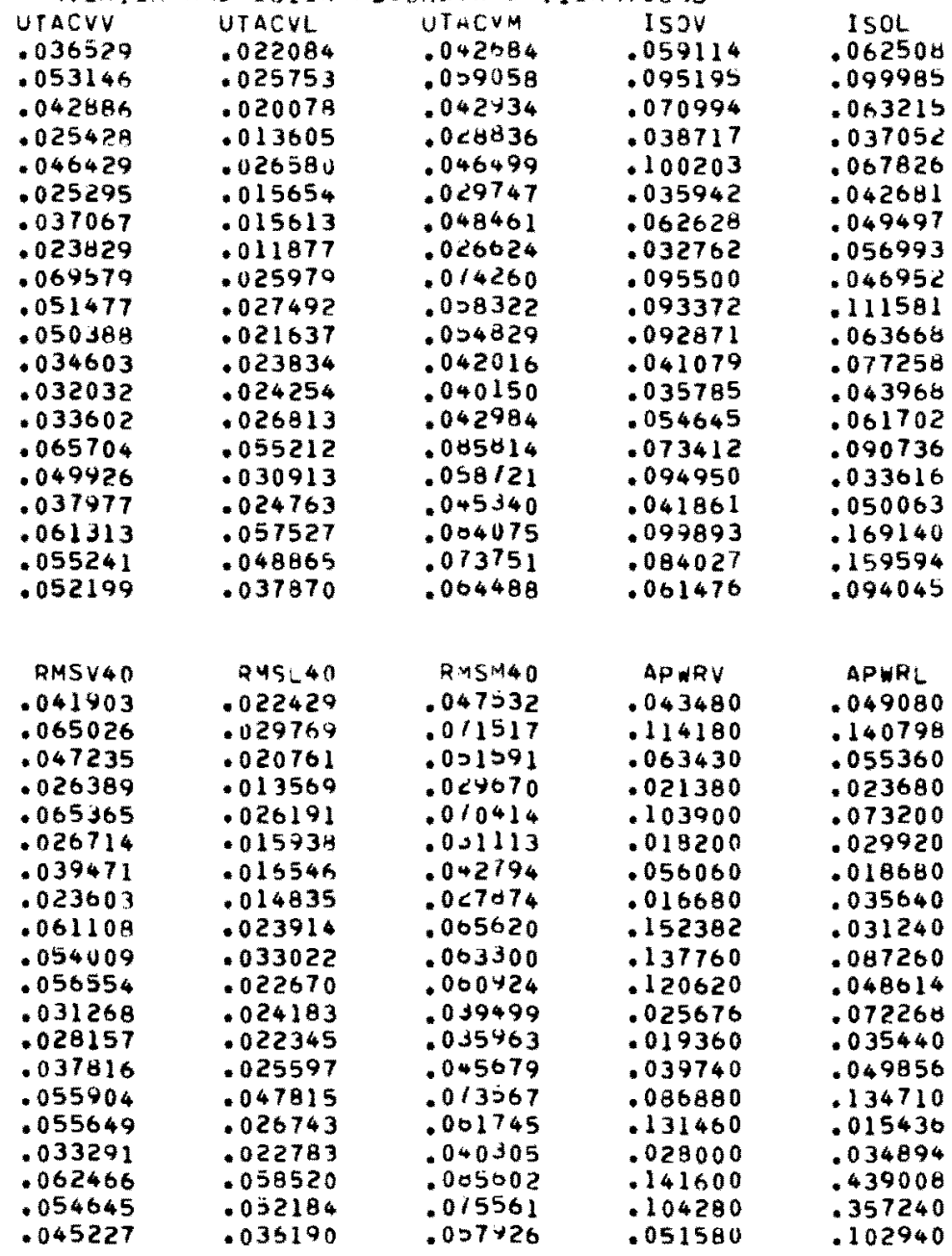

ISOM

.086012

.138056

.095059

.053590

.121000

.055794

.079827

.065733

.106418

.145494

- 112600

.047497

.056096

.082420

.116715

.100720

.065252

.196434

.180369

.112355

APWRT

.092560

.254974

.118800

.045040

.177100

.048120

.074720

.052320

.183620

.225040

.169240

.097940

.054800

.129340

.221580

.146900

.062900

.594868

.461340

.154500
HMS 1100

.042709

.065195

.050346

.027719

.007741

$.0278 B A$

.045043

.027379

.064502

.05 . $54 \mathrm{~A}$

.061165

.032272

.020826

.037689

058888

.057742

.035016

.064751

.055946

.047277

MPR

3.500000

2.330000

3.830000

4.420000

3.000000

4.250000

3.500000

4.170000

2.230000

2. 330000

3.000000

4.090000

4.070000

3.850000

2. 720000

2.540000

3.800000

2.200000

2.670000

3.390000 


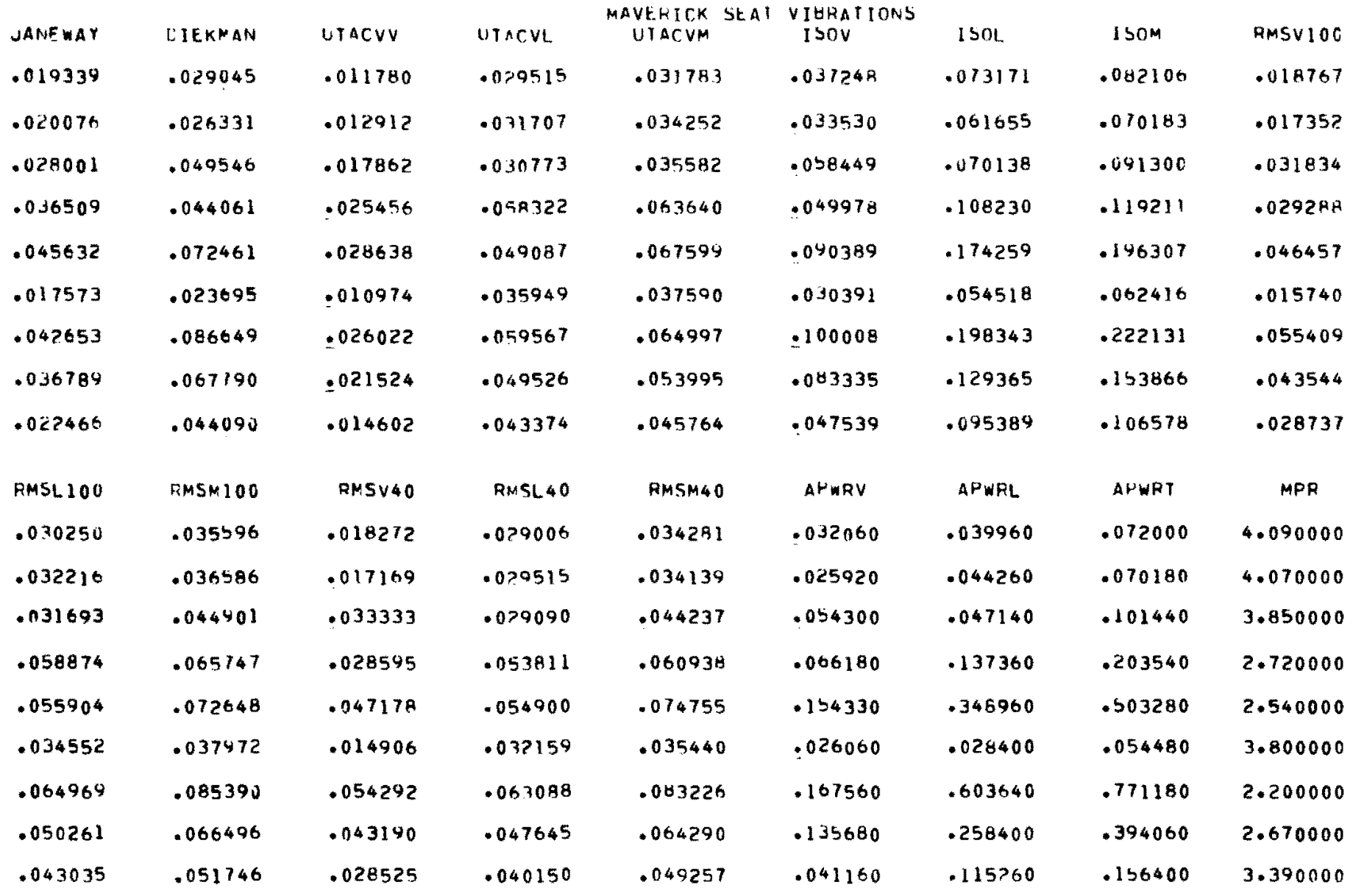


APPENDIX E.T

SCATTERGRAMS 

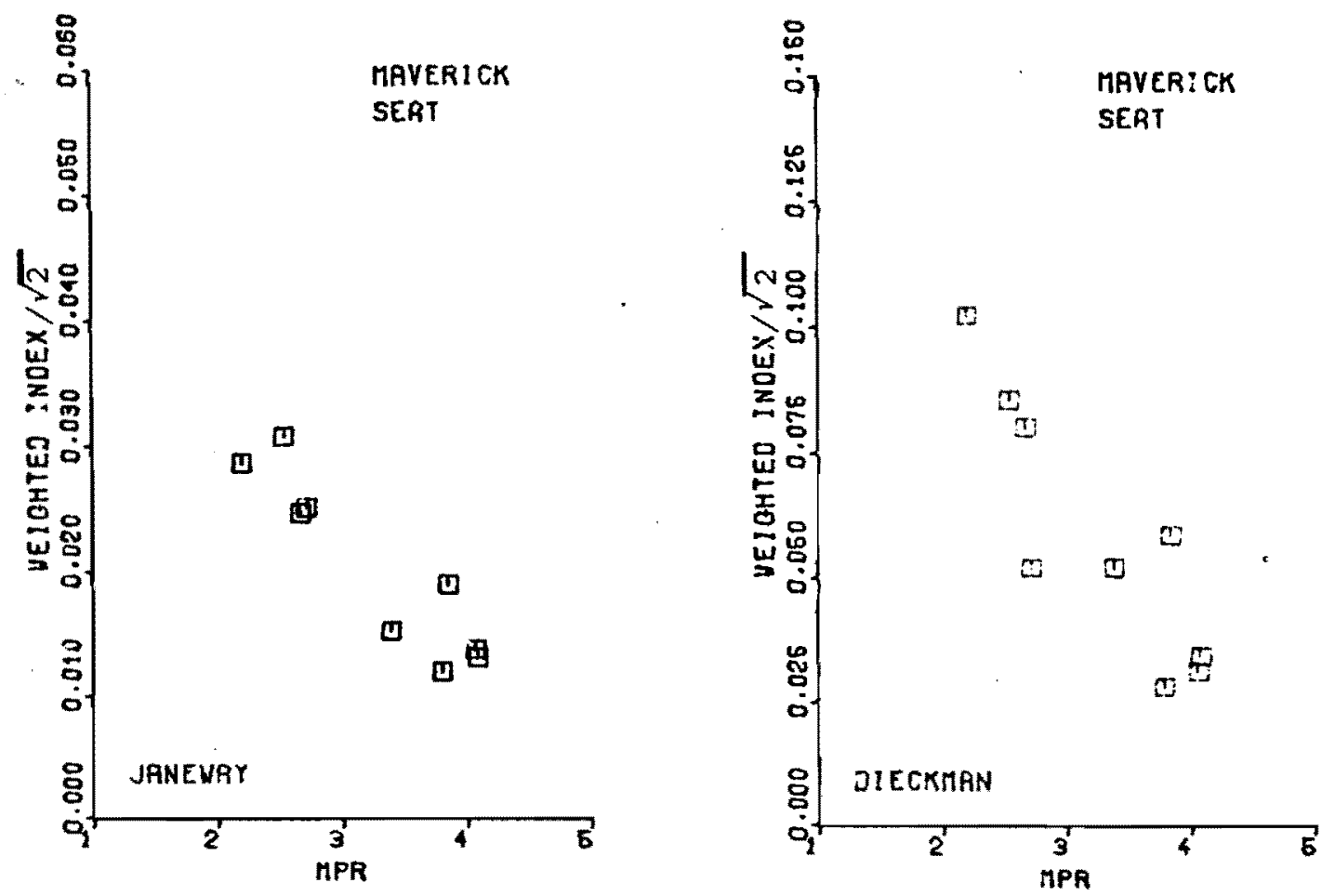
WEIGHTED INDICES VS. MEAN PERSONAL RATINGS
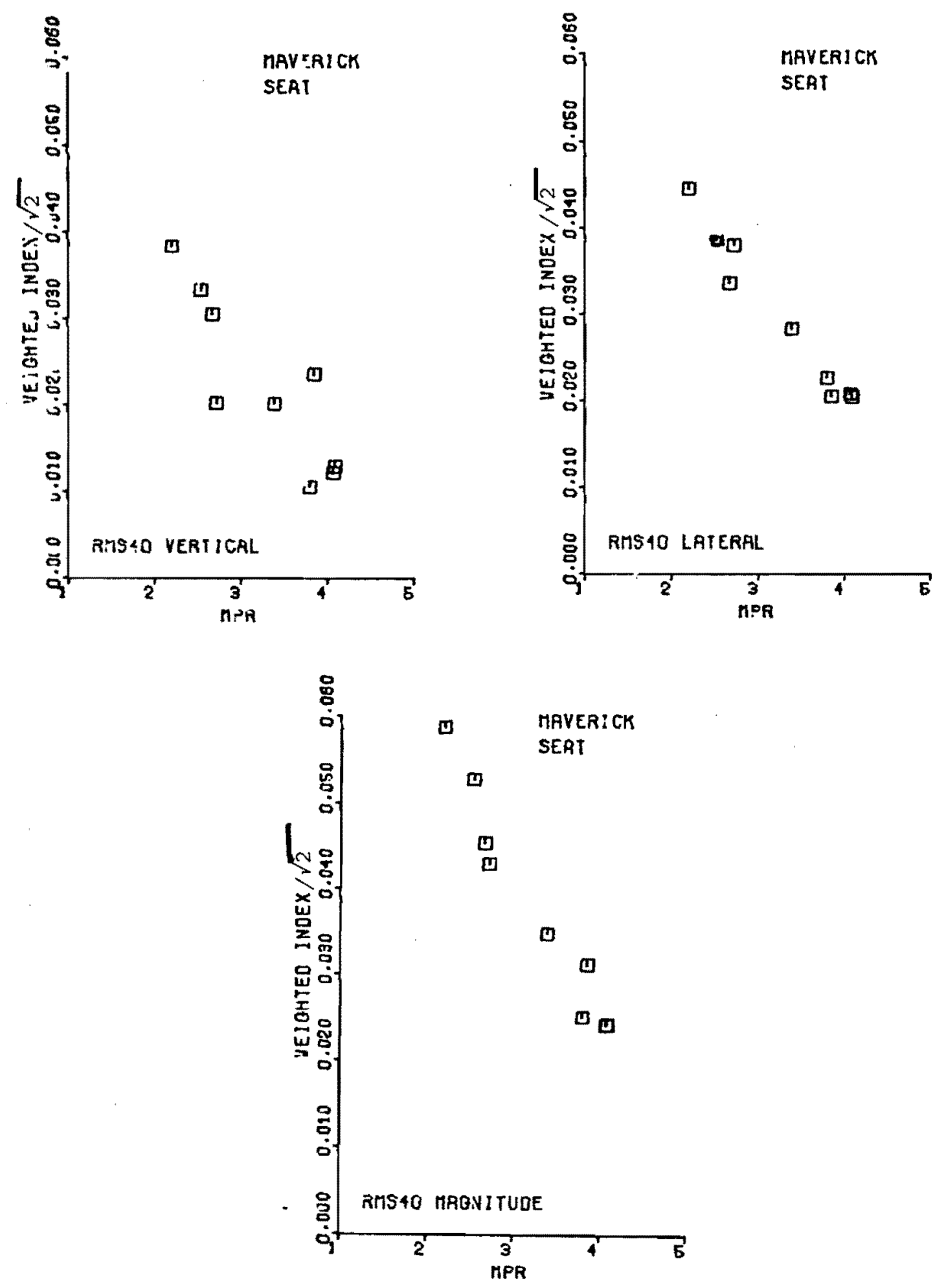
WEIGHTED INDICES VS. MEAN PERSONAL RATINGS

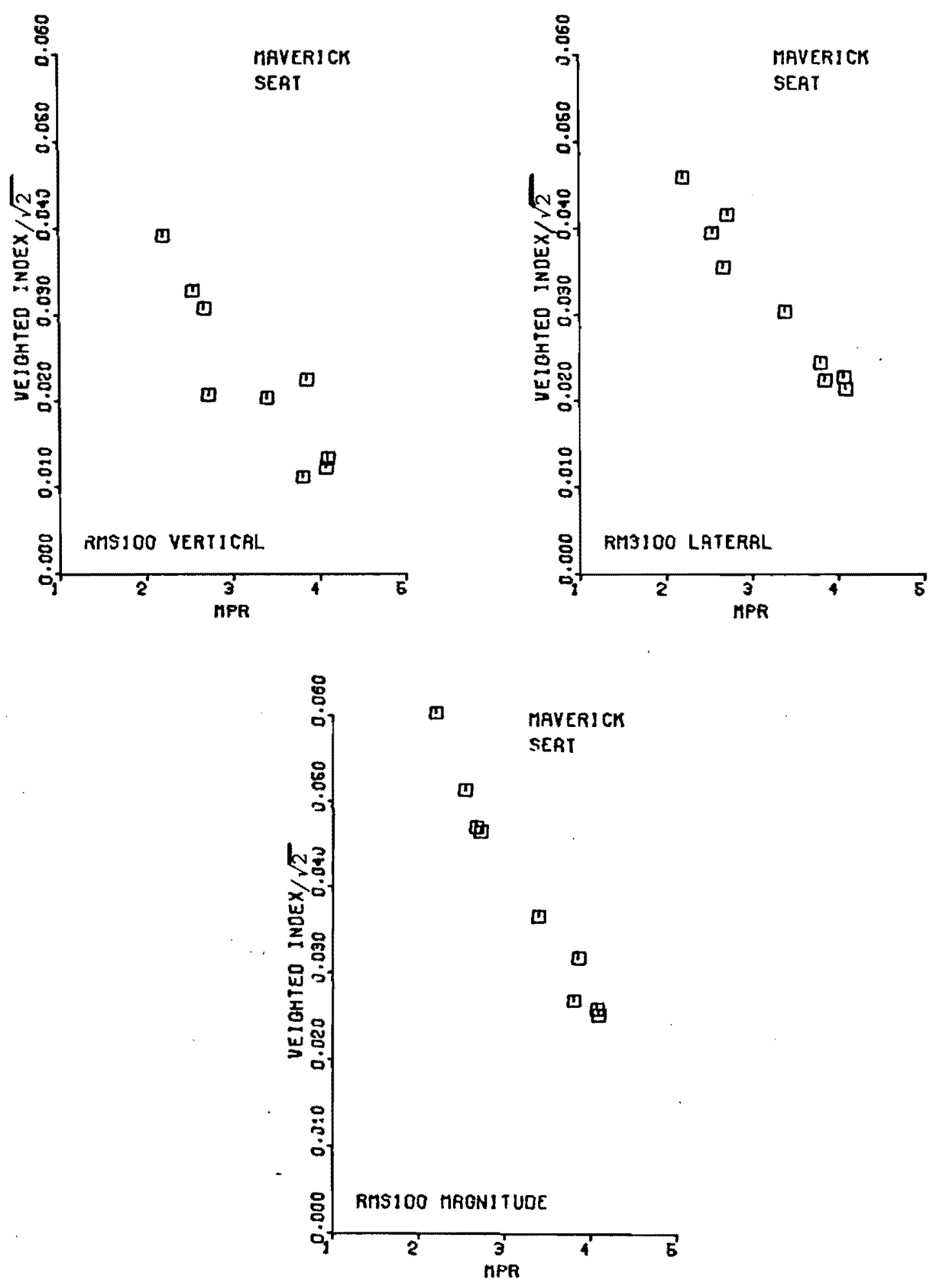


WEIGHTED INDICES VS. MEAN PERSONAL RATINGS

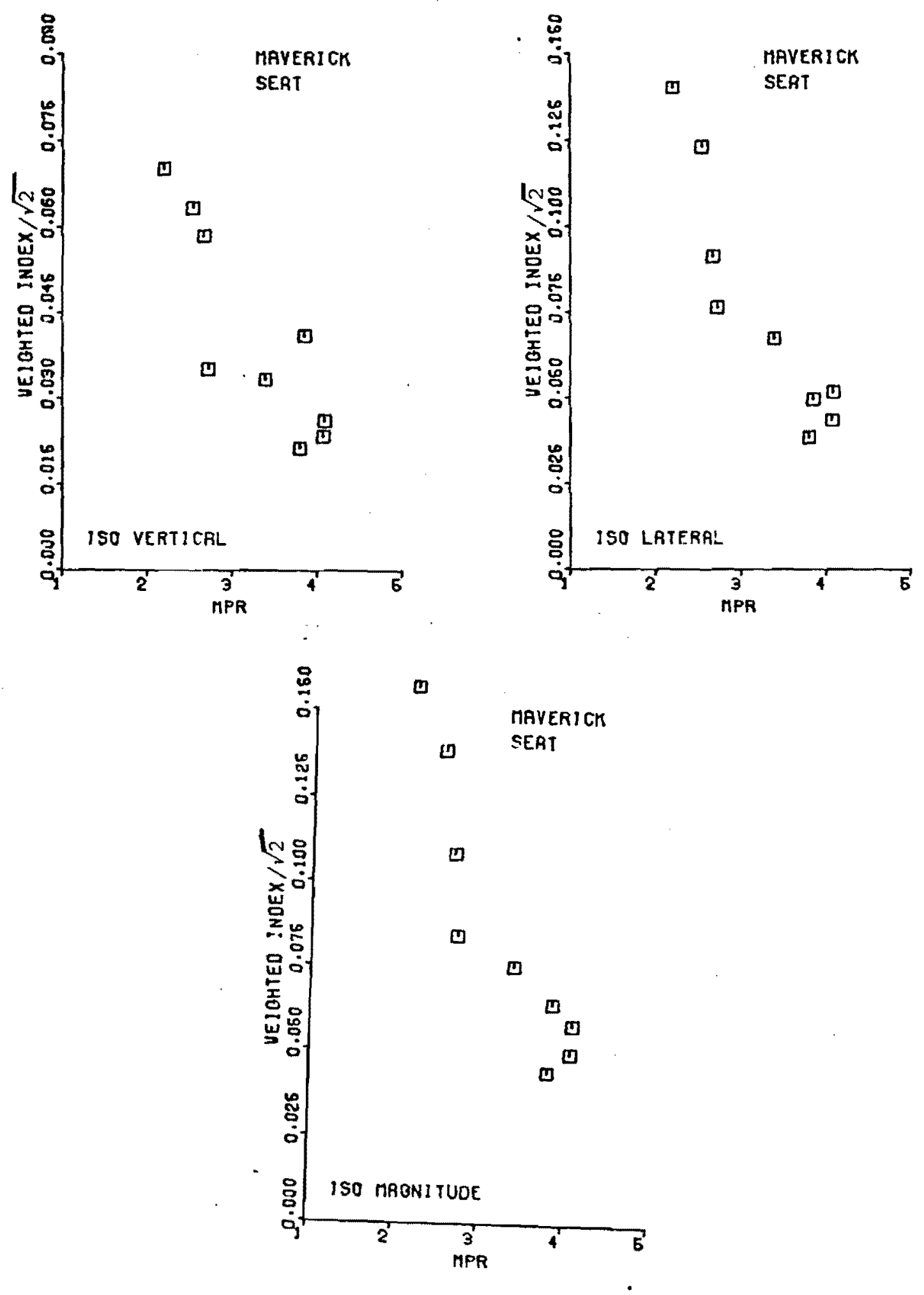


WEIGHTED INDICES VS. MEAN PERSONAL RATINGS
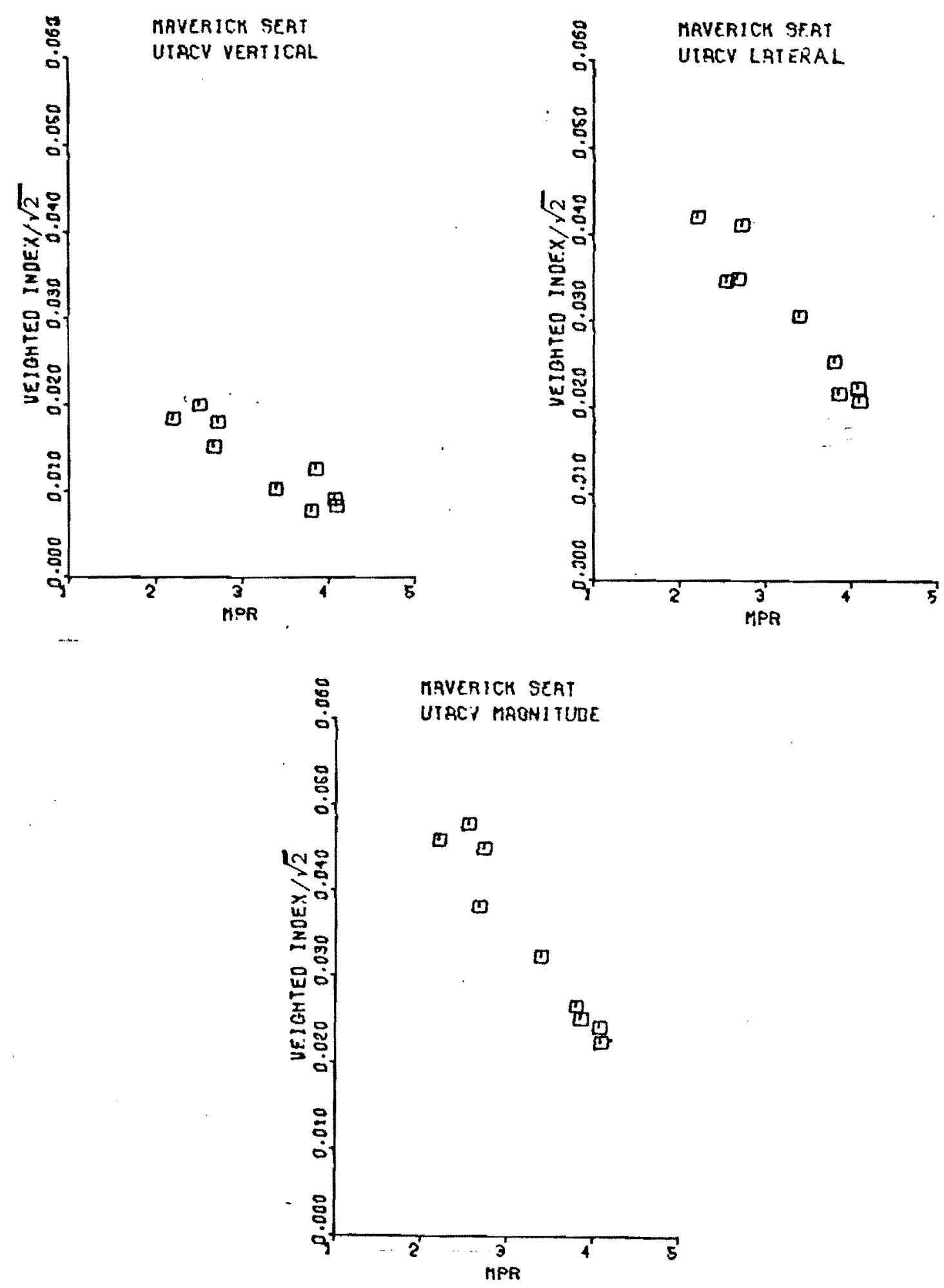


\section{WEIGHTED INDICES VS. MEAN PERSONAL RATINGS}
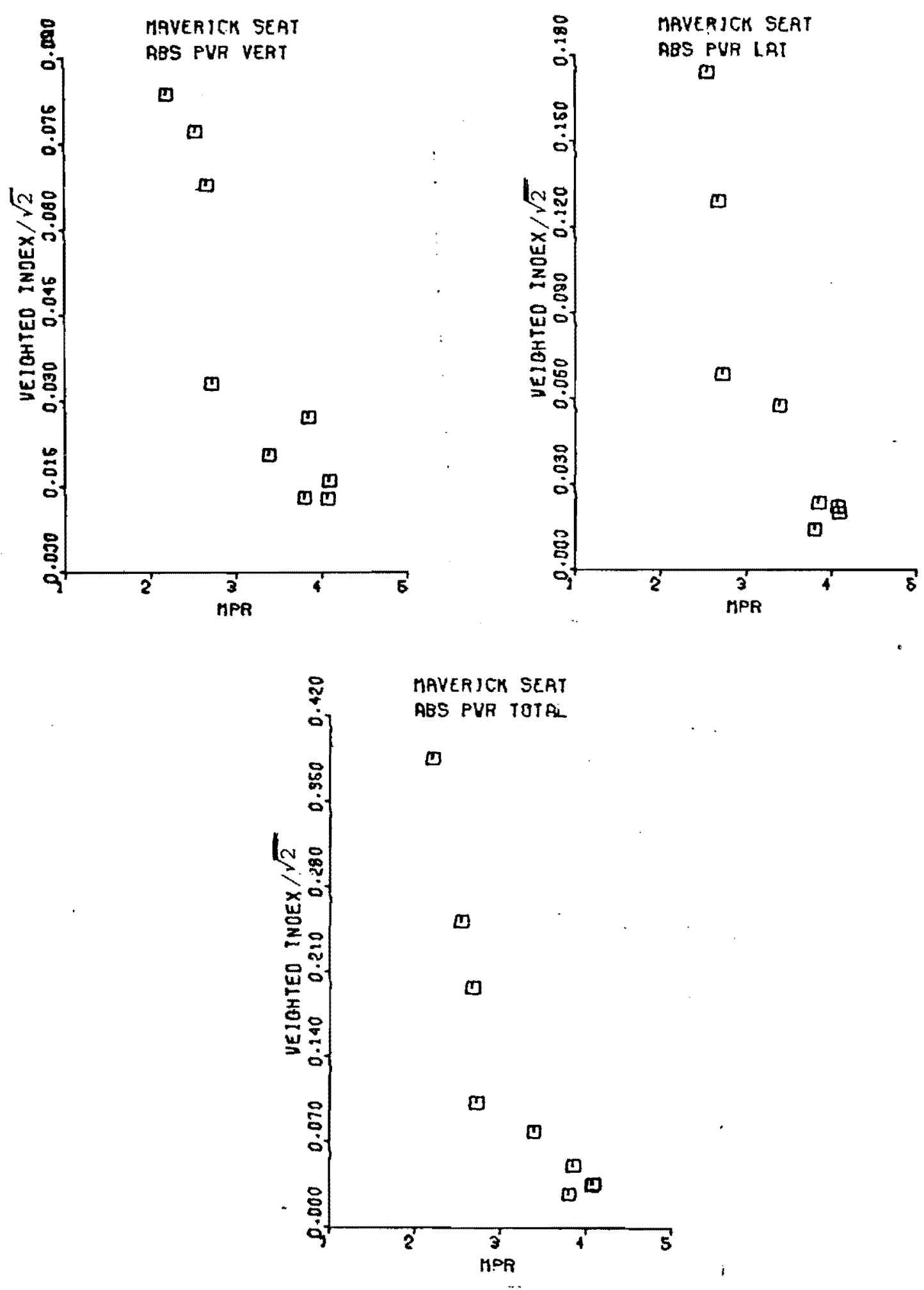


\section{WEIGHTED INDICES VS. MEAN PERSONAL RATINGS}
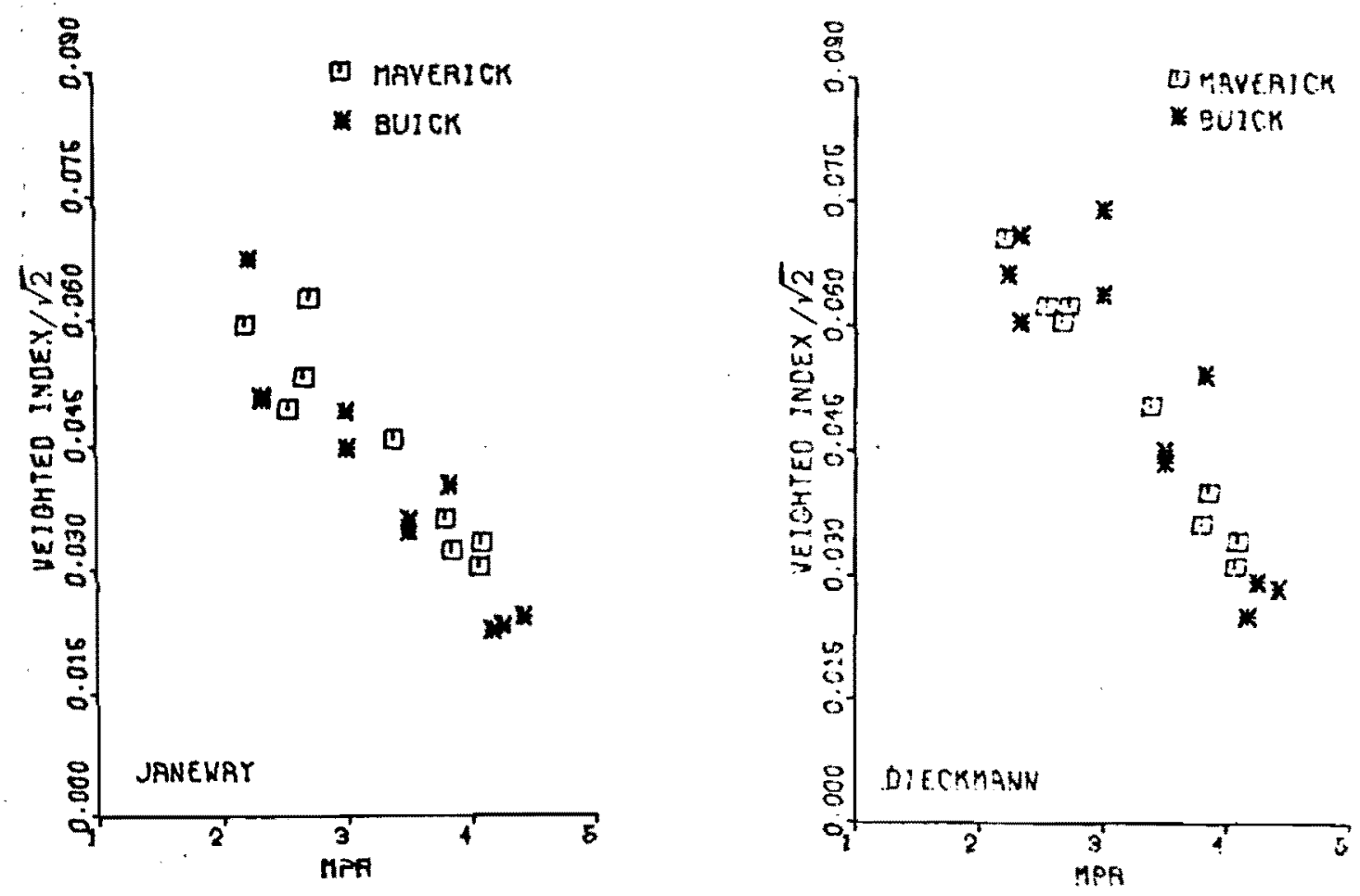
WEIGHTED INDICES VS. MEAN PERSONAL RATINGS
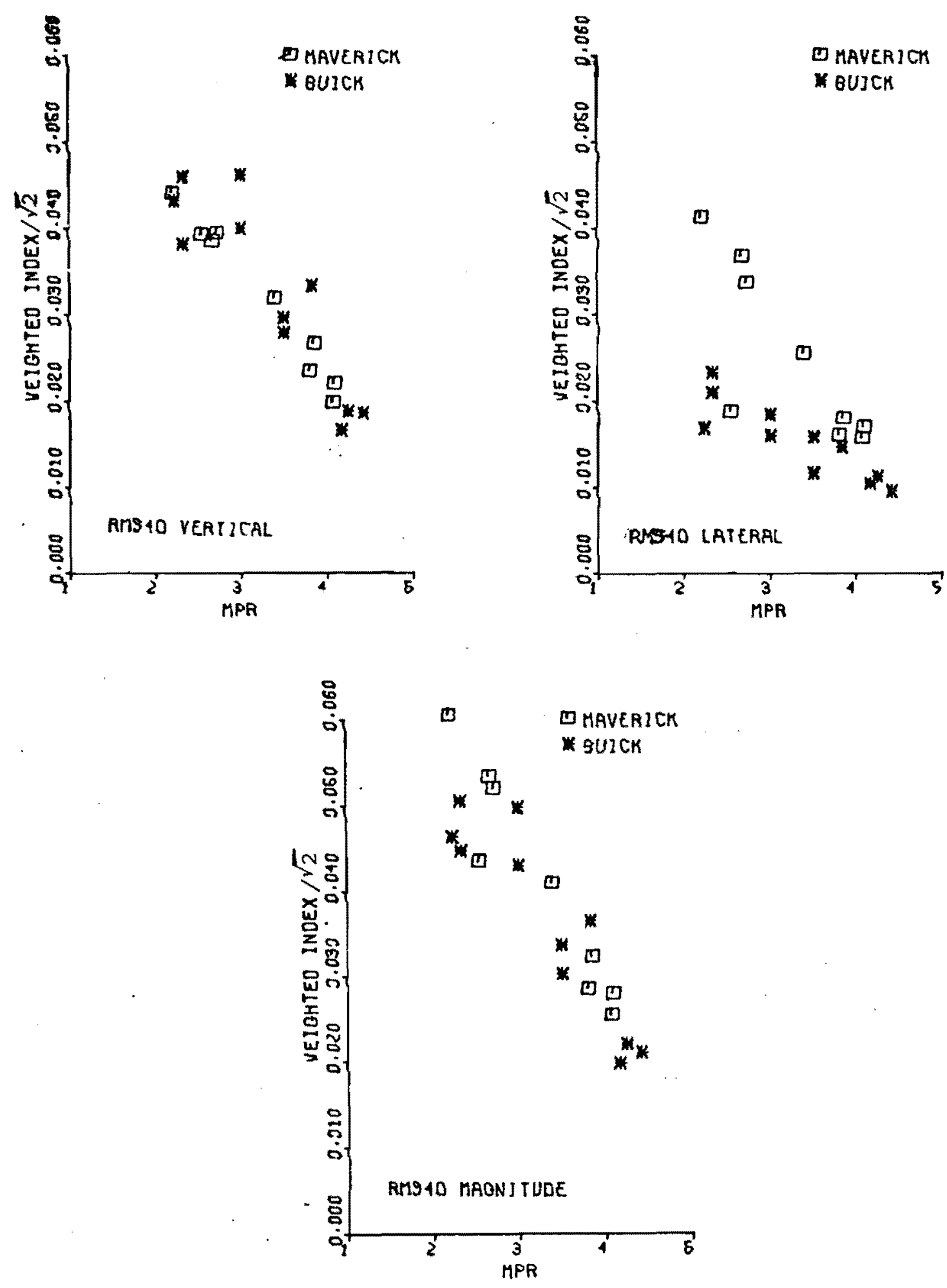
WEIGHTED INDICES VS. MEAN PERSONAL RATINGS
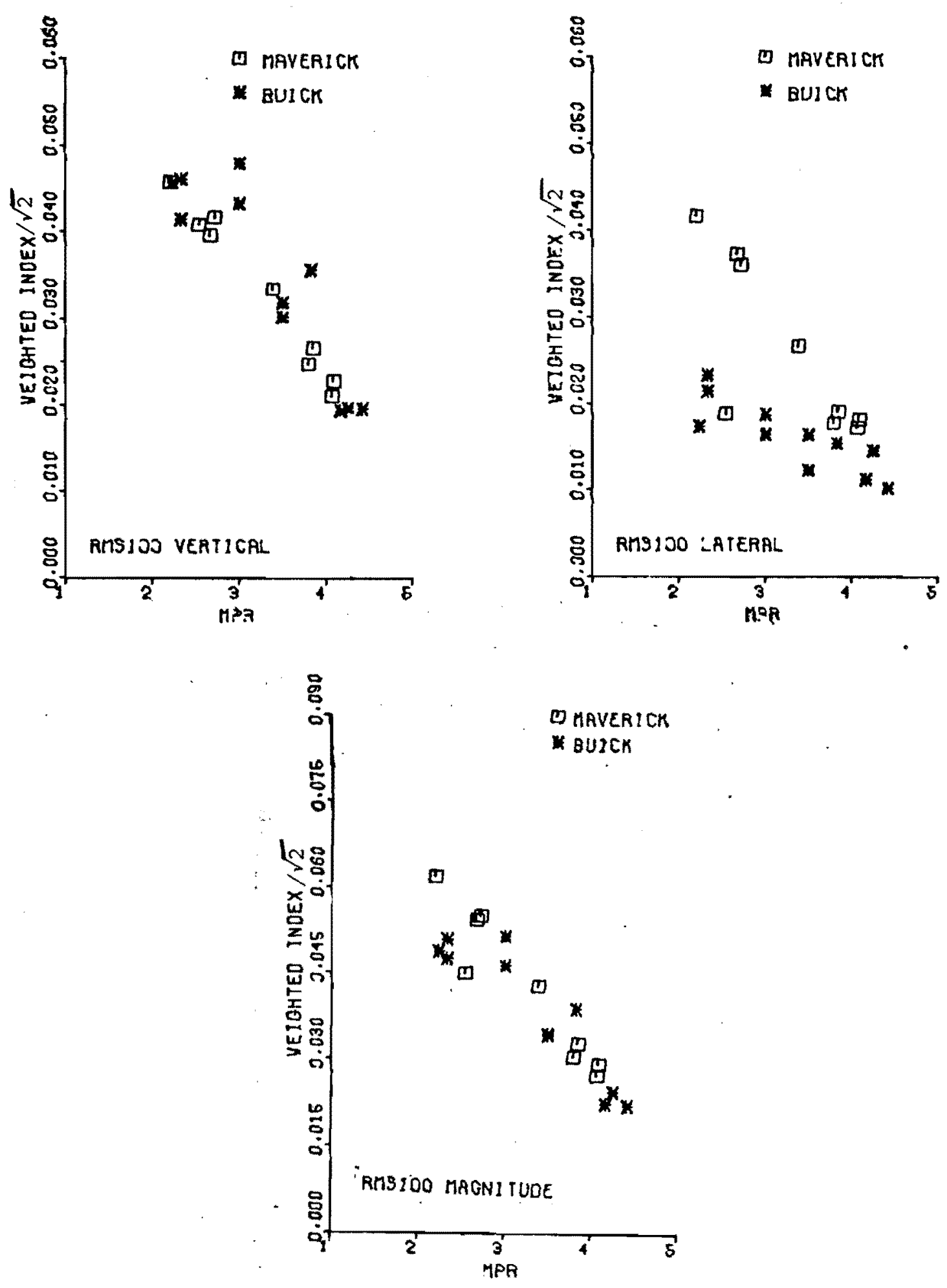
WEIGHTED INDICES VS. MEAN PERSONAL RATINGS
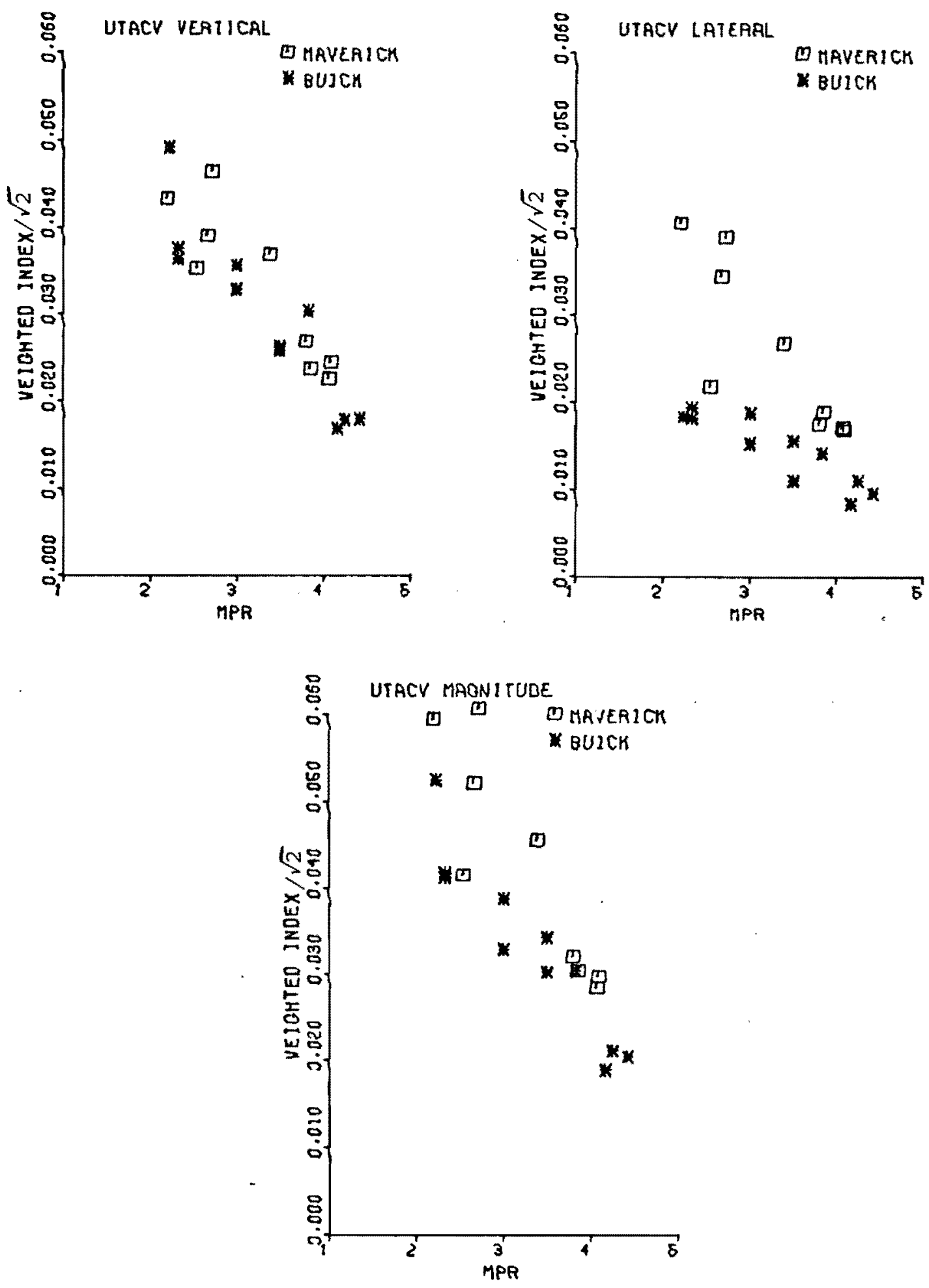
WEIGHTED INDICES VS. MEAN PERSONAL RATINGS
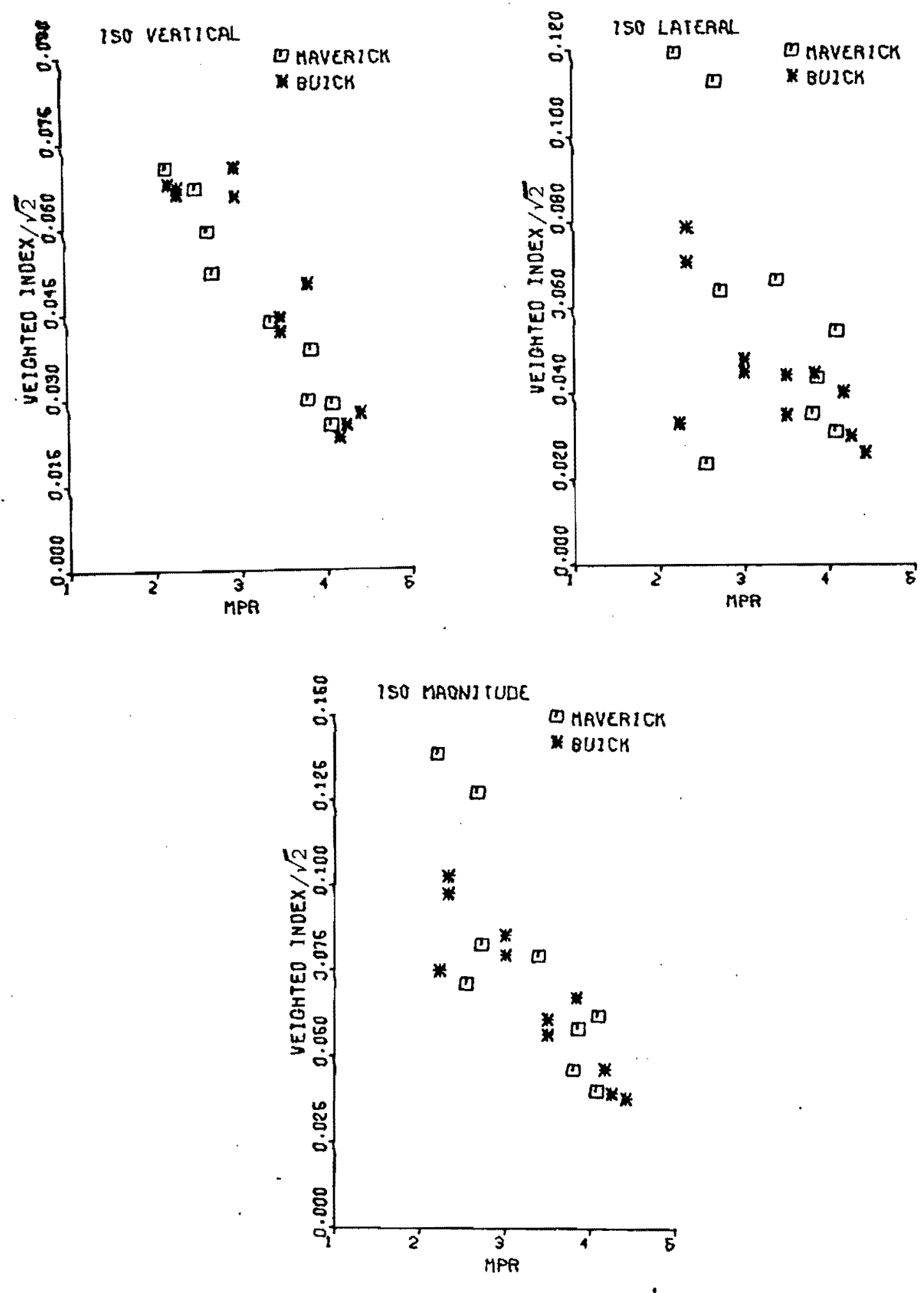
WEIGHTED INDICES VS. MEAN PERSONAL RATINGS
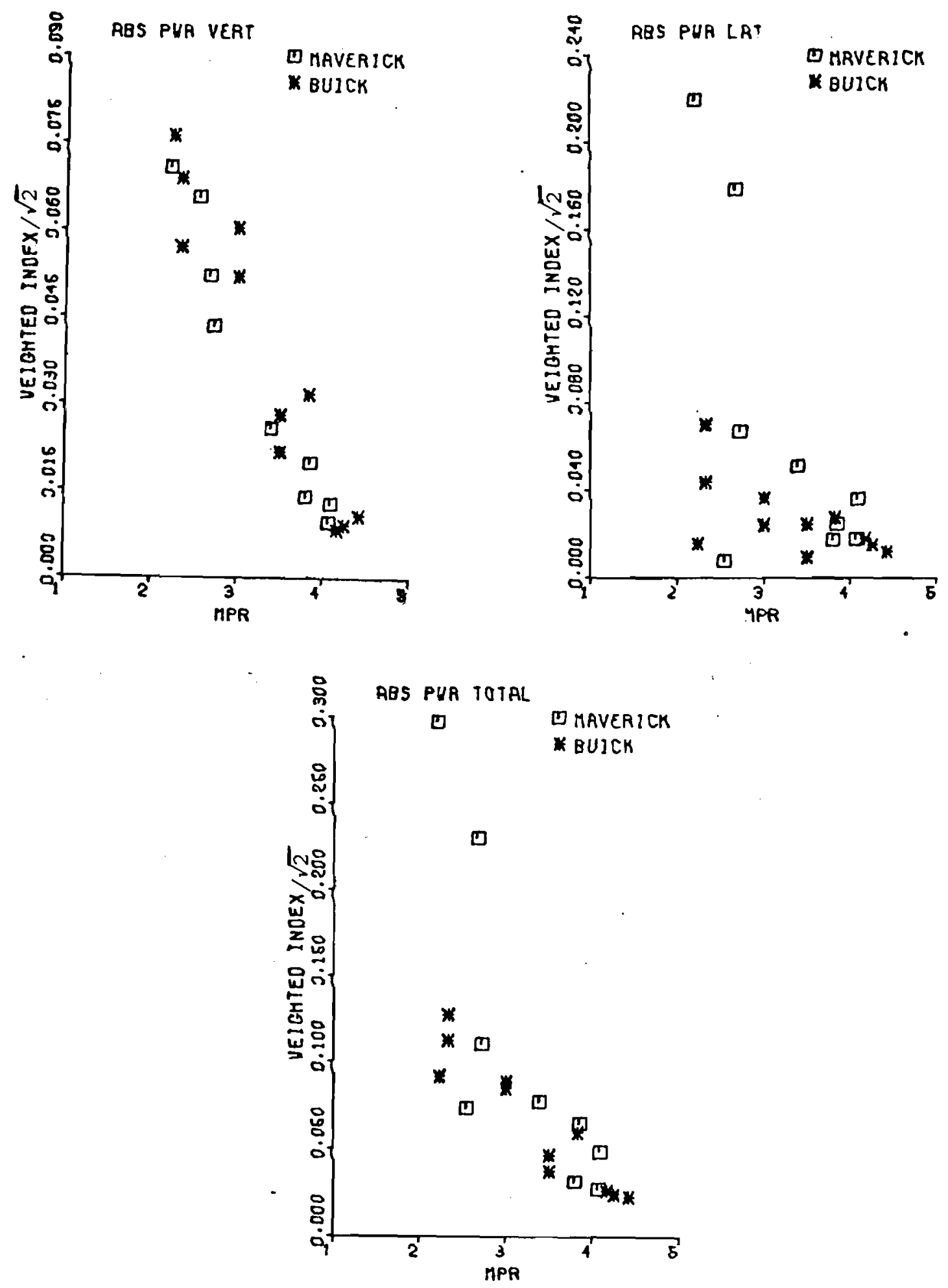
APPENDIX E.2

CORRELATION COEFFICIENTS AND RIDE INDEX EQUATION PARAMETERS FOR VARIOUS WEIGHTING FUNCTIONS 


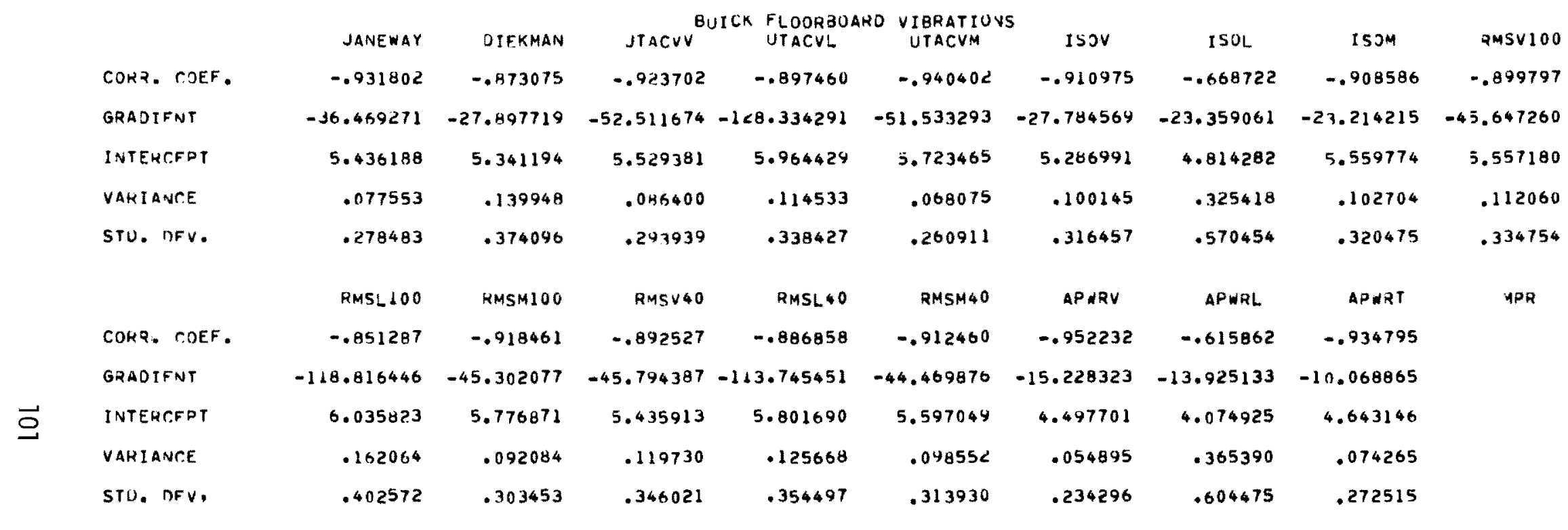




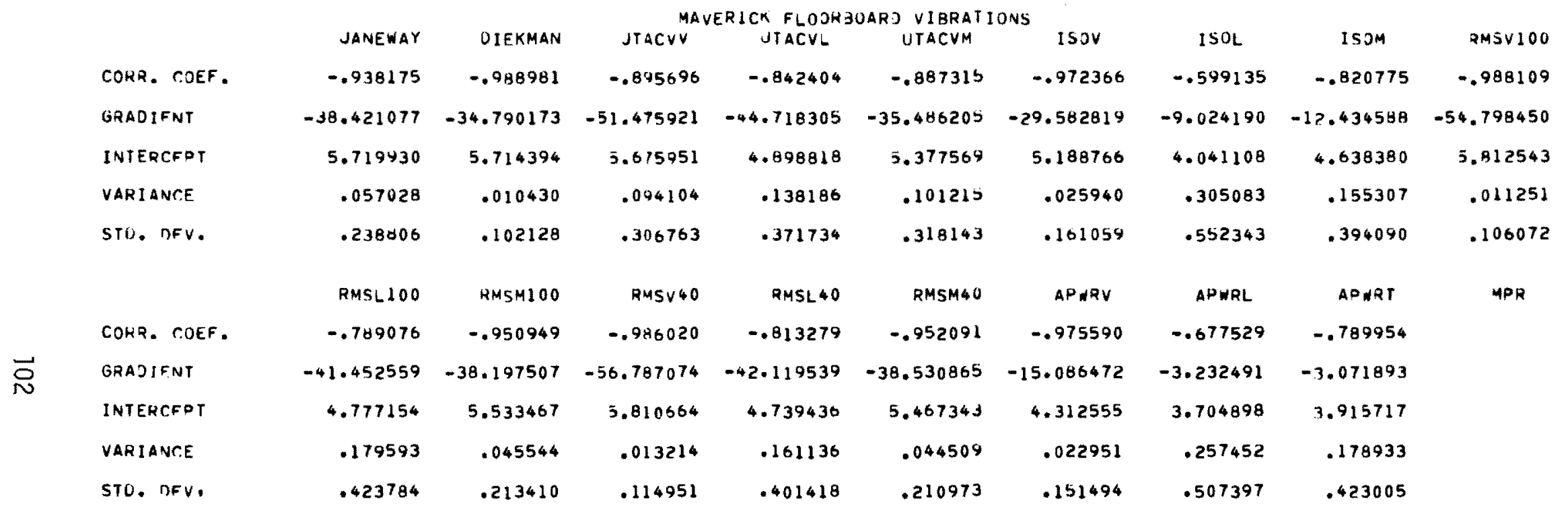




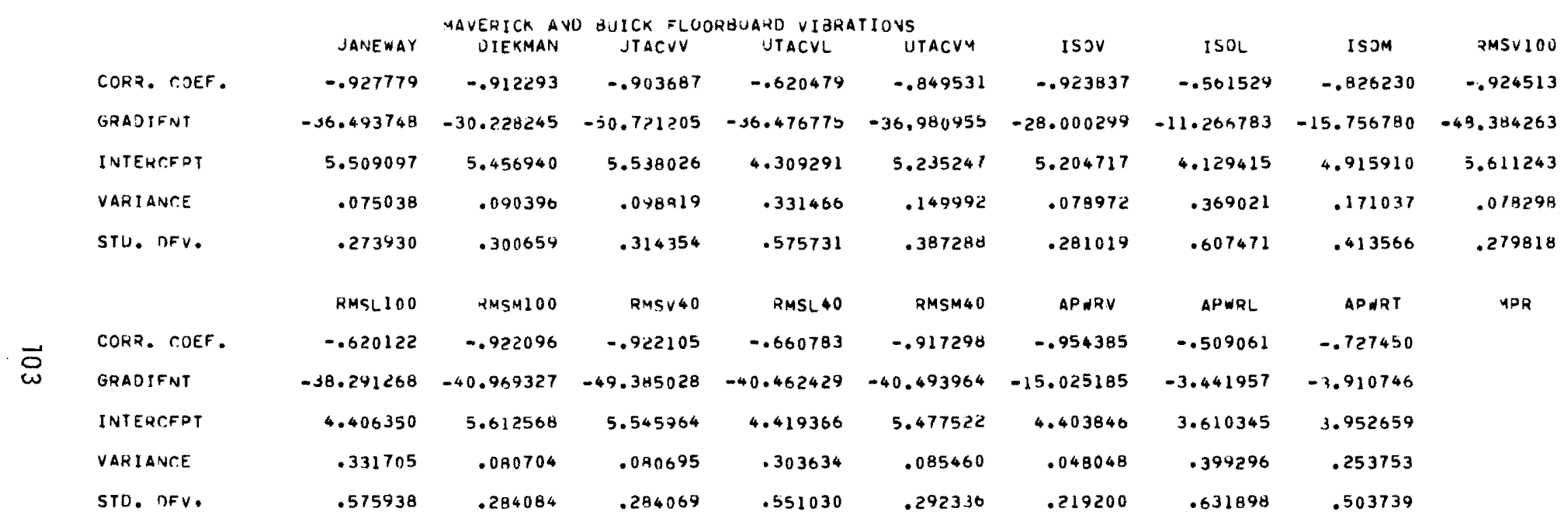




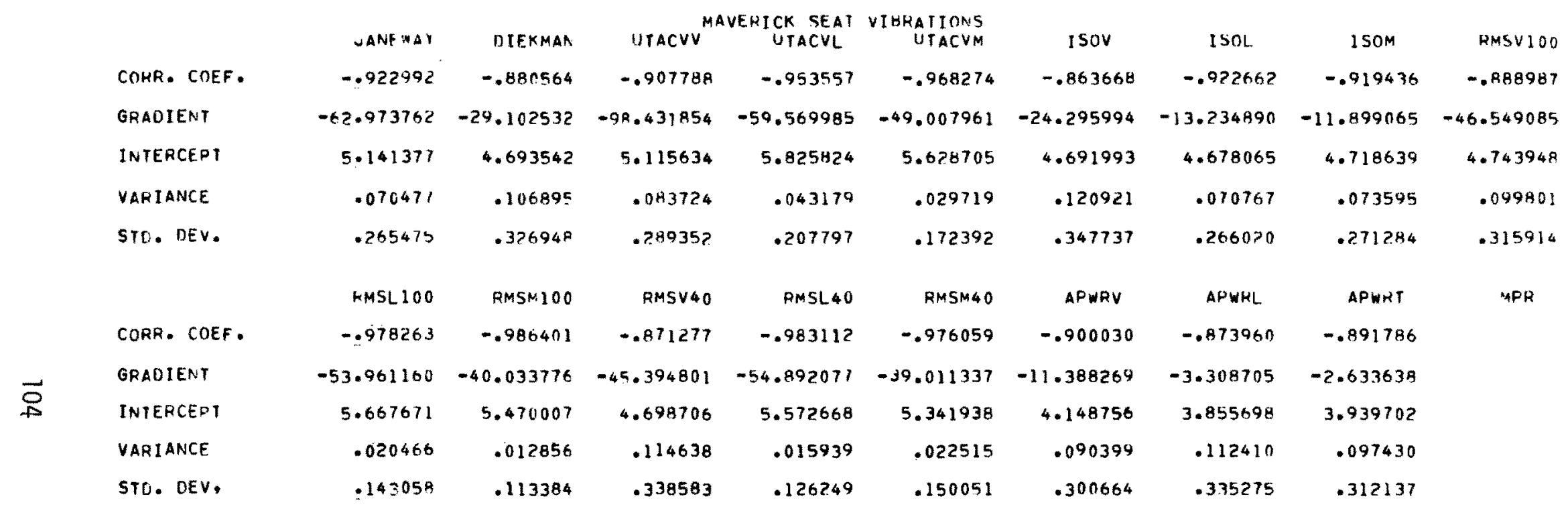




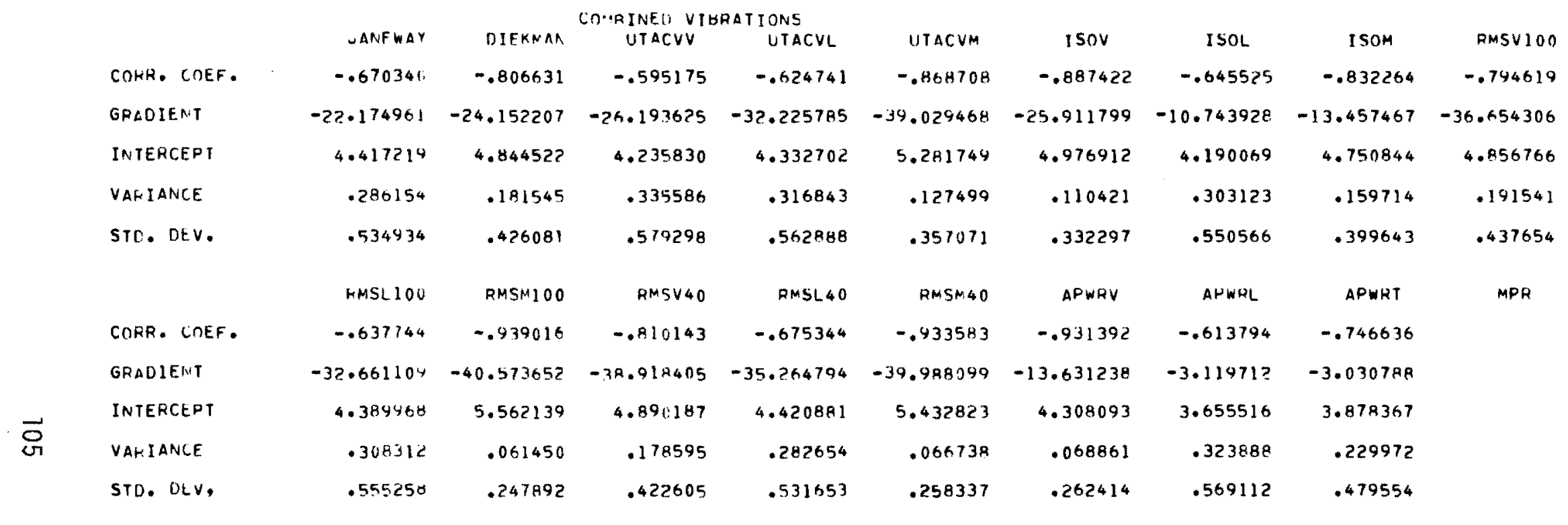


CRAIG C. SMITH was born in Provo, Utah, on May 1, 1944, the son of George Clinton and Metta Crawford Smith. He obtained his primary and secondary education in Blackfoot, Idaho, where he lived during most of his childhood years. He holds B.S.M.E. and M.S. degrees from Brigham Young University and a Ph.D. degree from the Massachusetts Institute of Technology. He has worked during summers for United States Steel Corporation and Bell Telephone Laboratories as well as having other shorter term industrial consulting experience. He has been involved in transportation related studies primarily related to vehicle and guideway dynamics beginning during his graduate work at M.I.T. He has been an Instructor at Brigham Young University, and is presently Assistant Professor of Mechanical Engineering at The University of Texas at Austin where he has been since September, 1973. He has taught courses covering a variety of topics, specializing in the areas of systems dynamics, control systems, machine design, and vibrations.

DAVID YOUNG McGEHEE was born in Houston, Texas, on Novenber 7, 1950, son of Dora Gaither McGehee and Edward Donald McGehee. Upon graduation from LaMarque High School, LaMarque, Texas, in 1969 he entered The University of Texas at Austin. He completed his Bachelor of Science degree in Mechanical Engineering in December 1973. He entered the Graduate School of The University of Texas at Austin in January 1974, and received his Master of Science degree in Decenber 1975. He is presently employed as a Design Engineer at General Dynamics Corporation in Fort Worth, Texas.

A. J. HEALEY was educated in England and came to the United States in 1966. His areas of interest lie in Dynamic System Modelling and Control. He taught at The Pennsylvania State University, M.I.T. and is currently Professor of Mechanical Engineering at The University of Texas at Austin. For the last three years he has been working in Ride Quality under contract to D.O.T. and heads the Ride Quality Group in the Council for Advanced Transportation at the University of Texas. He has authored several reports and papers in the area. He is currently the Secretary of the A.S.M.E. Automatic Control Division, and a Member of the New York Academy of Science. 
RESEARCH MEMORANDA PUBLISHED BY THE COUNCIL FOR ADVANCED TRANSPORTATION STUDIES

1 Human Response in the Evaluation of Modal Choice Decisions. C. Shane Davies, Mark Alpert, and W. Ronald Hudson, April 1973.

2 Access to Essential Services. Ronald Briggs, Charlotte Clark, James Fitzsimmons, and Paul Jensen, April 1973.

3 Psychological and Physiological Responses to Stimulation. D. W. Wooldridge, A. J. Healey, and R. O. Stearman, August 1973.

4 An Intermodal Transportation System for the Southwest: A Preliminary Proposal. Charles P. Zlatkovich, September 1973.

5 Passenger Travel Patterns and Mode Selection. Shane Davies, Mark Alpert, Harry Wolfe, and Rebecca Gonzalez, October 1973.

6 Segmenting a Transportation Market by Determinant Attributes of Modal Choice. Shane Davies and Mark Alpert, October 1973.

7 The Interstate Rail System: A Proposal. Charles P. Zlatkovich, December 1973.

8 Literature Survey on Passenger and Seat Modeling for the Evaluation of Ride Quality. Bruce Shanahan, Ronald Stearman, and Anthony Healey, November 1973.

9 The Definition of Essential Services and the Identification of Key Problem Areas. Ronald Briggs and lames Fitzsimmons, lanuary 1974.

10 A Procedure for Calculating Great Circle Distances Between Geographic Locations. J. Bryan Adair, March 1974.

11 MAPRINT: A Computer Program for Analyzing Changing Locations of Non-Residential Activities. Graham Hunter, Richard Dodge, and C. Michael Walton, March 1974.

12 A Method for Assessing the Impact of the Energy Crisis on Highway Accidents in Texas. E. L. Frome and C. Michael Walton, February 1975.

13 State Regulation of Air Transportation in Texas. Robert C. Means and Barry Chasnoff, April 1974.

14 Transportation Atlas of the Southwest. Charles P. Zlatkovich, S. Michael Dildine, Eugene Robinson, James W. Wilson, and J. Bryan Adair, June 1974.

15 Local Governmental Decisions and Land-Use Change: An Introductory Bibliography. W. D. Chipman, May 1974.

16 An Analysis of the Truck Inventory and Use Survey Data for the West South Central States. Michael Dildine, July 1974.

17 Towards Estimating the Impact of the Dallas-Fort Worth Regional Airport on Cround Transportation. William I. Dunlay and Lyndon Henry, September 1974.

18 The Attainment of Riding Comfort for a Tracked Air-Cushion Vehicle Through the Use of an Active Aerodynamic Suspension. Bruce Shanahan, Ronald Stearman, and Anthony Healey, September 1974.

19 Legal Obstacles to the Use of Texas School Buses for Public Transportation. Robert Means, Ronald Briggs, John E. Nelson, and Alan J. Thiemann, January 1975

20 Pupil Transportation: A Cost Analysis and Predictive Model. Ronald Briggs and David Venhuizen, April 1975.

21 Variables in Rural Plant Location: A Case Study of Sealy, Texas. Ronald Linehan, C. Michael Walton, and Richard Dodge, February 1975.

22 A Description of the Application of Factor Analysis to Land Use Change in Metropolitan Areas. John Sparks, Carl Gregory, and Jose Montemayor, December 1974.

23 A Forecast of Air Cargo Originations in Texas to 1990. Mary Lee Metzger Gorse, November 1974.

24 A Systems Analysis Procedure for Estimating the Capacity of an Airport: A Selected Bibliography. Chang-Ho Park, Edward V. Chambers, III, and William J. Dunlay, Jr., August 1975.

25 System 2000-Data Management for Transportation Impact Studies. Gordon Derr, Richard Dodge, and C. Michael Walton, September 1975.

26 Regional and Community Transportation Planning Issues: A Selected Bibliography. John Huddleston, Ronald Linehan, Abdulla Sayyari, Richard Dodge, C. Michael Walton, and Marsha Hamby, September 1975.

27 A Systems Analysis Procedure for Estimating the Capacity of an Airport: System Definition, Capacity Definition, and Review of Available Models. Edward V. Chambers, III, Tommy Chmores, William J. Dunlay, Ir., Nicolau D. F. Gualda, B. F. McCullough, ChangHo Park, and John Zaniewski, October 1975.

28 The Application of Factor Analysis to Land Use Change in a Metropolitan Area. John Sparks and Jose Montemayor, November 1975.

29 Current Status of Motor Vehicle Inspection: A Survey of Available Literature and Information. John Walter Ehrfurth and David A. Sands, December 1975 .

30 Executive Summary: Short Range Transit Improvement Study for The University of Texas at Austin. C. Michael Walton, May 1976. 31 A Preliminary Analysis of the Effects of the Dallas-Fort Worth Regional Airport on Surface Transportation and Land Use. Harry Wolfe, April 1974.

32 A Consideration of the Impact of Motor Common Carrier Service on the Development of Rural Central Texas. James Wilson, February 1975.

33 Modal Choice and the Value of Passenger Travel Time Literature: A Selective Bibliography. Shane Davies and Mark I. Alpert, March 1975.

34 Forecast of Air Cargo Originations in Arkansas, Louisiana, and Oklahoma to 1990. Deborah Goltra, April 1975.

35 Inventory of Freight Transportation in the Southwest/Part IV: Rail Service in the Dallas-Fort Worth Area. Charles P. Zlatkovich, Mary L. Gorse, Edward N. Kasparik, and Dianne Y. Priddy, April 1975.

36 Forecast of Waterborne Commerce Handled by Texas Ports to 1990. Stuart Metz Dudley, April 1975.

37 Forecast of Refinery Receipts of Domestic Crude Oil from Pipelines in the West South Central States to 1990. Mary L. Gorse, Dianne Y. Priddy, and Deborah J. Goltra, April 1975.

38 A Feasibility Study of Rail Piggyback Service Between Dallas-Fort Worth and San Antonio. Edward N. Kasparik, April 1975.

39 Land Value Modeling in Rural Communities. Lidvard Skorpa, Richard Dodge, and C. Michael Walton, June 1974.

40 Toward Computer Simulation of Political Models of Urban Land Use Change. Carl Gregory, August 1975.

41 A Multivariate Analysis of Transportation Improvements and Manufacturing Growth in a Rural Region. Ronald linehan, C. Michael Walton, and Richard Dodge, October 1975.

42 A Transit Demand Model for Medium-Sized Cities. John Shortreed, December 1975. 


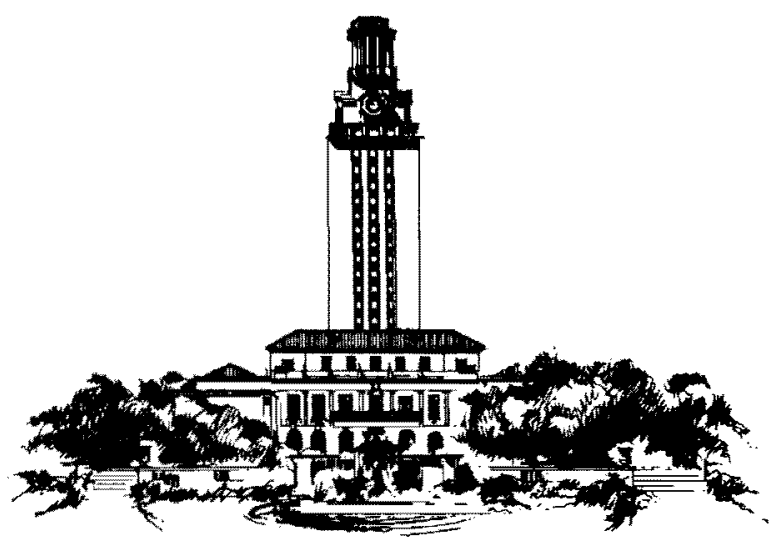

Council for Advanced Transportation Studies

THE UNIVERSITY OF TEXAS AT AUSTIN 